hep-ph/9603307

\title{
Physics Beyond the Standard Model f
}

\author{
J. W. F. Valle \\ Instituto de Física Corpuscular - C.S.I.C. \\ Departament de Física Teòrica, Universitat de València \\ 46100 Burjassot, València, SPAIN
}

\begin{abstract}
We discuss some of the signatures associated with extensions of the Standard Model related to the neutrino and electroweak symmetry breaking sectors, with and without supersymmetry. The topics include a basic discussion of the theory of neutrino mass and the corresponding extensions of the Standard Model that incorporate massive neutrinos; an overview of the present observational status of neutrino mass searches, with emphasis on solar neutrinos, as well the as cosmological data on the amplitude of primordial density fluctuations; the implications of neutrino mass in cosmological nucleosynthesis, non-accelerator, as well as in high energy particle collider experiments. Turning to the electroweak breaking sector, we discuss the physics potential for Higgs boson searches at LEP200, including Majoron extensions of the Standard Model, and the physics of invisibly decaying Higgs bosons. We discuss the minimal supersymmetric Standard Model phenomenology, as well as some of the laboratory signatures that would be associated to models with $\mathrm{R}$ parity violation, especially in $\mathrm{Z}$ and scalar boson decays.
\end{abstract}

${ }^{a}$ These lectures were given at the VIII Jorge Andre Swieca Summer School (Rio de Janeiro, February 1995) and at the $V$ Taller Latinoamericano de Fenomenologia de las Interacciones Fundamentales (Puebla, Mexico, October 1995). For convenience the material of the lectures has been reorganized and updated.

${ }^{b}$ E-mail: valle@flamenco.uv.es, URL http://neutrinos.uv.es 


\section{Contents}

\begin{tabular}{llr}
\hline 1 & Introduction & 1
\end{tabular}

1.1 Standard Model Basics . . . . . . . . . . . . . . . . . . . . . . 2

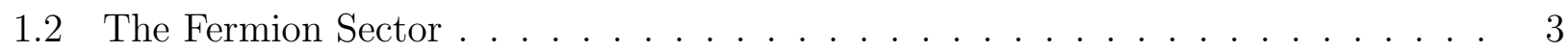

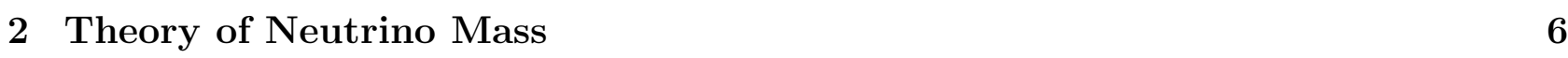

2.1 Dirac and Majorana Masses . . . . . . . . . . . . . . . . . . . . 6

2.2 Neutrinos in The Standard Model and Beyond . . . . . . . . . . . . . . . . . . . 10

2.3 Leptonic Charged Currents . . . . . . . . . . . . . . . . . . . . . . 12

2.4 Leptonic Neutral Current . . . . . . . . . . . . . . . . . . . . . . . 15

2.5 Constraints on neutrino-NHL mixing . . . . . . . . . . . . . . . . . . . . 16

2.6 Phenomenological Implications $\ldots \ldots \ldots$

2.7 Laboratory Limits on Neutrino Masses . . . . . . . . . . . . . . . . . . . . . . . 19

2.8 The Cosmological Density Limit . . . . . . . . . . . . . . . . . . . . . . 21

2.9 The Cosmological Nucleosynthesis Limit . . . . . . . . . . . . . . . . . . . . . . 22

2.10 Hints for Neutrino Masses . . . . . . . . . . . . . . . . . . . . . . . 24

2.10 .1 Dark Matten . . . . . . . . . . . . . . . . . . . . . 24

2.10 .2 Solar Neutrino Experiments . . . . . . . . . . . . . . . . . . . . 25

2.10 .3 Atmospheric Neutrinos . . . . . . . . . . . . . . . . . . . 30

2.11 Models Reconciling Present Hints. . . . . . . . . . . . . . . . . . . . . . 30

2.11 .1 Three Almost Degenerate Neutrinos . . . . . . . . . . . . . . . . . . . . . 30

2.11 .2 Three Active plus One Sterile Neutring . . . . . . . . . . . . . . . . . 31

$2.11 .3 \mathrm{MeV}$ Tau Neutring . . . . . . . . . . . . . . . . . 31

3 Electroweak Symmetry Breaking: The Higgs Sector 32

3.1 Standard Model Higgs $\ldots \ldots \ldots$. . . . . . . . . . . . . . . . . 32

3.2 Majoron Models . . . . . . . . . . . . . . . . . . . . . . . 35

3.2 .1 Simplest Model . . . . . . . . . . . . . . . . . . . . . . . 36 
3.2 .2 Two Scalar Higgs Doublet Extensions . . . . . . . . . . . . . . . . . . . . 38

\begin{tabular}{|ll}
\hline & Electroweak Symmetry Breaking: Supersymmetry
\end{tabular}

4.1 The MSSM . . . . . . . . . . . . . . . . . . . . . . . . . . . . . . . 41

4.2 The MSSM Higgs Sector . . . . . . . . . . . . . . . . . . . . . . . . . 44

4.3 Limits on SUSY Particles . . . . . . . . . . . . . . . . . . . . . . . . . . . . . . 46

4.4 Explicit R Parity Violation . . . . . . . . . . . . . . . . . . . . . . . . . 50

4.5 Spontaneous R Parity Violation . . . . . . . . . . . . . . . . . . . . . 51

4.6 The Scalar Sector in the Model . . . . . . . . . . . . . . . . . . . . . . . . . . . 56

4.7 Implications of Spontaneous R Parity Breaking . . . . . . . . . . . . . . . . . . 59

4.8 R Parity Breaking Scalar Boson Decays . . . . . . . . . . . . . . . . . . . . . . . 63

$\begin{array}{llr}5 \text { Outlook } & 63\end{array}$ 


\section{Introduction}

There is a wide consensus that, although very successful wherever it has been tested, our present Standard Model leaves open too many fundamental issues in particle physics to be an ultimate theory of nature. These lectures will focus on two of these that have to do with the understanding of what lies behind the mechanism of mass generation in general, and with the masses and the properties of neutrinos in particular.

A basic assumption of the Standard Model is the Higgs mechanism, which is introduced as a way to generate the masses of all the fundamental particles. This mechanism implies the existence of a fundamental scalar boson [1]. If such an elementary boson exists it is widely believed that some stabilising principle - e.g. supersymmetry (SUSY) - should be operative at the electroweak scale in order to explain the stability of its mass scale against quantum corrections associated with physics at super-high energies. The observed joining of the three gauge coupling strengths as they are evolved from the presently accessible energies up to a common scale of $\sim 10^{16}$ $\mathrm{GeV}$ provides circumstantial evidence that supersymmetry seems to set in somewhere around $M_{S U S Y} \sim 10^{3} \mathrm{GeV}$. Probing the details of this rich structure constitutes one of the main goals in the agenda of the next generation of elementary particle colliders.

Another fundamental issue in the Standard Model refers to the properties of neutrinos. Apart from being a theoretical puzzle, in the sense that there is no principle that dictates that neutrinos are massless, as postulated in the Standard Model, nonzero masses may in fact be required in order to account for the data on solar and on atmospheric neutrinos, as well as for an explanation of the dark matter in the universe. The possible detection of nonzero neutrino masses would have far-reaching implications for the understanding of fundamental issues in particle physics, astrophysics, as well as for the large-scale structure of our universe.

The above two different types of extensions of the Standard Model may be inter-connected. An example is provided by supersymmetric models with spontaneously broken $\mathrm{R}$ parity, which necessarily imply non-vanishing neutrino masses. As a result in some of these models there are novel processes that could be observed at high energy colliders whose magnitude would be correlated to the mass of the neutrinos. One interesting aspect of these models is that

they may affect the physics of the electroweak sector in such a remarkable way, that can be probed in various present and future experiments, both at accelerator as well as underground installations, as we will describe. 
Table 1: Multiplets of the Standard Model

\begin{tabular}{|c|c|}
\hline$W$ & $\mathrm{~S} U(3) \otimes S U(2) \otimes U(1)$ \\
\hline$B$ & $(1,3,0)$ \\
\hline$\phi$ & $(1,1,0)$ \\
\hline$\ell_{a}$ & $(1,2,1)$ \\
$e_{a}^{c}$ & $(1,1,2)$ \\
$Q_{a}$ & $(3,2,1 / 3)$ \\
$u_{a}^{c}$ & $(\overline{3}, 1,-4 / 3)$ \\
$d_{a}^{c}$ & $(\overline{3}, 1,2 / 3)$ \\
\hline
\end{tabular}

\section{$1.1 \quad$ Standard Model Basics}

The Standard Model is a Yang-Mills theory based on the $S U(3) \otimes S U(2) \otimes U(1)$ gauge group, and described by the field representations in table 1, where all fermions are left-handed. The fundamental constituents of matter - quarks and leptons - interact mainly due to the exchange of the gauge bosons. The Lagrangian describing the Standard Model can be found in many textbooks and reviews [2]. In order to comply with the fact that the weak interaction is mediated by massive vector bosons, the $W$ and the $Z$, the gauge symmetry has to be broken. The best way to do this in a way consistent with renormalizability and unitarity is through the nonzero vacuum expectation value (VEV)

$$
v / \sqrt{2}=\left\langle\phi^{0}\right\rangle
$$

of the neutral component of a complex Higgs scalar doublet $\phi$

$$
\phi=\left(\begin{array}{c}
\phi^{+} \\
\phi^{0}
\end{array}\right) \text {. }
$$

Through the term $|D \phi|^{2}$, where $D$ denotes the covariant derivative that follows from the quantum numbers in table 1 , this gives mass to all gauge bosons except one. This corresponds to the surviving $U(1)_{\text {em }}$ gauge symmetry corresponding to the unbroken QED electro-magnetic gauge invariance, whose generator is the electric charge, identified as

$$
Q=T_{3}+Y / 2
$$


where $T_{3}$ and $Y$ denote the diagonal isospin and hyper-charge generators. Diagonalizing the neutral gauge boson mass matrix leads to a mixing between the gauge bosons $B_{\mu}$ and $W_{3 \mu}$ associated to the generators $Y$ and $T_{3}$,

$$
\begin{gathered}
Z_{\mu}=\cos \theta_{W} W_{3 \mu}+\sin \theta_{W} B_{\mu} \\
A_{\mu}=-\sin \theta_{W} W_{3 \mu}+\cos \theta_{W} B_{\mu}
\end{gathered}
$$

where

$$
\theta_{W}=\arctan \frac{g^{\prime}}{g}
$$

is the electroweak mixing angle, $g$ and $g^{\prime}$ are the $S U(2)$ and $U(1)$ gauge coupling strengths, respectively. The massless vector boson $A_{\mu}$ is the photon, while $Z_{\mu}$ has a mass

$$
m_{Z}=\frac{g v}{2 \cos \theta_{W}}
$$

while the charged gauge bosons $W^{ \pm}=\frac{1}{\sqrt{2}}\left(W_{1} \mp i W_{2}\right)$ have a mass

$$
m_{W}=\frac{g v}{2} .
$$

The $W$ and the $Z$ gauge bosons have been discovered in 1983 by the UA1 and UA2 experiments at CERN. The properties of the $Z$ have now been very precisely determined by the LEP experiments [3], while those of the $W$ mostly come from UA2 and the Fermilab CDF and D0 experiments [⿴囗十 . The measured gauge boson mass values agree well with the electroweak theory predictions, once radiative corrections are included.

\subsection{The Fermion Sector}

The Standard Model contains three generations of quarks and leptons $(a=1,2,3)$, also given in table 1. Different generations are identical in their gauge properties, but differ in mass. The fermion assignment is clearly not invariant under parity, since only the left handed fermions (not the anti-fermions) transform as $S U(2)$ doublets. As a result the fermions can not be given gauge invariant masses. Fermion masses arise from Yukawa-type interactions with the Higgs doublet scalar,

$$
h_{u a b} u_{a}^{c} Q_{b} \tau_{2} \phi^{*}+h_{d a b} d_{a}^{c} Q_{b} \phi+h_{e a b} e_{a}^{c} \ell_{b} \phi
$$

which generate masses to all charged fermions $f=u, d, e$

$$
h_{f} v / \sqrt{2}=M_{f}
$$

after electroweak breaking. Thus in the Standard Model the weak gauge boson as well as the charged fermion masses are generated spontaneously, by the Higgs mechanism. Note that the 
coupling matrices $h_{u}, h_{d}$ and $h_{e}$ are arbitrary complex non-symmetric matrices in generation space. This gives rise to a vast proliferation of parameters in the model. The understanding the pattern of fermion masses and mixing is one of the main challenges of the theory and clearly lies beyond the Standard Model.

The currents describing the interaction of quarks and leptons with the standard electroweak gauge fields can be written simply from the gauge covariant derivatives that follow from table 1. For example the terms in the Lagrangian describing the interaction of quarks and leptons with the charged gauge bosons $W^{ \pm}$are

$$
\frac{i g}{\sqrt{2}} W_{\mu}^{+} \sum_{a=1}^{n} \bar{u}_{L a} \gamma_{\mu} d_{L a}+\frac{i g}{\sqrt{2}} W_{\mu}^{+} \sum_{a=1}^{n} \bar{\nu}_{L a} \gamma_{\mu} e_{L a}+H . C .
$$

where $u_{a}$ and $d_{a}$ are weak-eigenstate quarks $(u, c, t$ and $d, s, b)$, and $\nu_{a}$ and $e_{a}$ denote the three neutrinos and the three charged leptons, $e, \mu, \tau$. The contact with the old four-Fermi weak interaction theory is established through

$$
\frac{G_{F}}{\sqrt{2}}=\frac{g^{2}}{8 m_{W}^{2}} .
$$

where $G_{F}$ is the Fermi constant, well determined from $\mu$ decay. In addition to the charged current weak interactions, quarks and leptons also have neutral current weak interactions, described by

$$
\frac{i g^{\prime}}{\sin \theta_{W}} Z_{\mu} \sum_{A} \bar{\Psi}_{A} \gamma_{\mu}\left(T_{3}-\sin ^{2} \theta_{W} Q\right) \Psi_{A}
$$

where $g \sin \theta_{W}=g^{\prime} \cos \theta_{W}=e=\sqrt{4 \pi \alpha}$ and $\Psi_{A}$ denotes an arbitrary helicity fermion belonging to any of the multiplets in table 1. By including electroweak radiative corrections in the charged and neutral currents one obtains a very good description of all known weak interaction processes. In particular, all known neutral current phenomena are well described by a single parameter, the electroweak mixing angle $\theta_{W}$. The success of the LEP experiments in the precise determination of the electroweak parameters has been so remarkable that just the internal consistency of the various measurements is sufficient to provide a very good determination of the mass of the top quark $m_{t}=180 \pm 14 \mathrm{GeV}$, with the error largely due to the lack of knowledge of the Higgs boson mass [3]. This is in excellent agreement with the direct measurement at Fermilab [4].

Similarly, the total $Z$ decay width, as well as its partial widths have been precisely measured by the LEP collaborations, leaving little room for new physics. Of special interest to us is the measurement of the invisible $Z$ width [5]

$$
\Gamma_{i n v}^{Z}=499.9 \pm 2.5 \mathrm{MeV}
$$

which can be translated into a measurement of the effective number of Standard Model neutrino generations. This places a very stringent constraint on models of neutrino mass where lepton number is a global symmetry spontaneously broken at low energies (see section 3.2 below). 
Let us now turn to a discussion of the flavour structure of the charged current weak interactions. First we consider the case of quarks. In order to determine the form of the charged current we first diagonalize separately the quark mass matrices $M_{u}$ and $M_{d}$

$$
\begin{aligned}
& U_{R u}^{\dagger} M_{u} U_{L u}=\operatorname{diag}\left(m_{u}, m_{c}, m_{t}\right) \\
& U_{R d}^{\dagger} M_{d} U_{L d}=\operatorname{diag}\left(m_{d}, m_{s}, m_{b}\right)
\end{aligned}
$$

Since these matrices can not in general be made diagonal in the same basis, it follows that the charged current quark weak interactions, rewritten in terms of the mass-eigenstate quarks $u_{i}$ and $d_{i}$, take the following form

$$
\frac{i g}{\sqrt{2}} W_{\mu}^{+} \bar{u}_{L i} \gamma_{\mu} V_{i j} d_{L j}+H . C .
$$

where $V=U_{L u}^{\dagger} U_{L d}$. Some of the parameters in the unitary matrix $V$ are not physical, since they can be eliminated by appropriate quark redefinitions. For $n$ generations $V_{i j}$ involves a set of

$$
n(n-1) / 2
$$

mixing angles $\theta_{i j}$ and

$$
n(n-1) / 2-(n-1)
$$

CP violating phases $\phi_{i j}$. They can be brought to the following useful form [6, 7]

$$
V=\omega_{0}(\alpha) \prod_{i<j}^{n} \omega_{i j}\left(\eta_{i j}\right) \omega_{0}^{\dagger}(\alpha)
$$

where $\theta_{i j}=\left|\eta_{i j}\right|$ and $\omega_{0}=\operatorname{diag}\left(e^{i \alpha_{i}}\right)$ is a diagonal matrix of phases, for example,

$$
\omega_{12}\left(\eta_{12}\right)=\left(\begin{array}{ccccc}
c_{12} & e^{i \phi_{12}} s_{12} & 0 & 0 & \ldots \\
-e^{-i \phi_{12}} s_{12} & c_{12} & 0 & 0 & \ldots \\
0 & 0 & 1 & 0 & \ldots \\
0 & 0 & 0 & 1 & \ldots \\
\ldots & \ldots & \ldots & \ldots & \ldots
\end{array}\right)
$$

For the simplest 2 generation case one sees that the $2 \times 2$ mixing matrix $V$ is completely determined by one parameter, the Cabibbo angle. In this case CP is necessarily conserved. As first noted by Kobayashi and Maskawa [8], for three generations there are 3 mixing angles and a single phase that describes CP violation in the Standard Model. The first parametrizations of the three generation mixing matrix $V$ were given in ref. [8] and [9]. A useful truncated form was introduced by Wolfenstein [10]. So far this scheme has been proven sufficient to account for the observed pattern of quark mixing and CP violation in the Standard Model [5]. However, 
one lacks a fundamental theory of mass and flavour and, as a result, the parameters in $V$ are all undetermined and must be extracted from experiment. Note that the CP phases in eq. (18) are not directly observable: only special combinations (as many as given by our counting) which are invariant under quark rephasings have physical meaning. For 3 generations this $\mathrm{CP}$ violating combination is unique.

Finally, note that the weak neutral current of the mass-eigenstate quarks has a trivial flavour structure, i.e. it is given by the identity matrix both in the weak and in the mass-eigenstate basis. This important property is usually referred to as the Glashow-Iliopoulos-Maiani (GIM) mechanism [11]. It accounts for the absence of flavour changing neutral current mediated processes so far observed. Any deviations from this rule would indicate the presence of physics beyond the Standard Model.

\section{Theory of Neutrino Mass}

The first basic concept one needs to develop in order to make a theoretical discussion of neutrino masses is that of Majorana and Dirac masses.

\subsection{Dirac and Majorana Masses}

The most basic kinematical concept necessary to describe the physics of massive neutrinos is that of a Majorana fermion, and how it relates with the more familiar one of a Dirac fermion. A massive Majorana fermion has just half of the number of degrees of freedom of a conventional massive spin 1/2 Dirac fermion and corresponds to the lowest representation of the Lorentz group. The basic Lagrangian describing such particle is [6, 12

$$
L_{M}=-i \rho^{\dagger} \sigma_{\mu} \partial_{\mu} \rho-\frac{m}{2} \rho^{T} \sigma_{2} \rho+H . C .
$$

and is expressed in terms of a 2-component spinor $\rho$. In eq. (20) $\sigma_{i}$ are the usual $2 \times 2$ Pauli matrices and $\sigma_{4}=-i I$, with $I$ being the identity matrix. Pauli's metric convention

for Minkowski coordinates is used, where the dot product of two four vectors is $a . b \equiv a_{\mu} b_{\mu} \equiv$ $\vec{a} \cdot \vec{b}+a_{4} b_{4}$, where $a_{4}=i a_{0}$. Under a Lorentz transformation, $x \rightarrow \Lambda x$, the spinor $\rho$ transforms as $\rho \rightarrow S(\Lambda) \rho\left(\Lambda^{-1} x\right)$ where $S$ obeys

$$
S^{\dagger} \sigma_{\mu} S=\Lambda_{\mu \nu} \sigma_{\nu}
$$

The kinetic term in eq. (20) is clearly invariant. Similarly, the mass term is invariant, as a result of unimodular property $\operatorname{det} S=1$. However, since it is not invariant under a phase transformation

$$
\rho \rightarrow e^{i \alpha} \rho
$$


the Majorana mass term is only admissible in a theory of electrically neutral fermions such as neutrinos, or other more exotic fermions of the type present in supersymmetric theories, e.g., neutral gauginos and Higgsinos.

The equation of motion following from eq. (20) is

$$
-i \sigma_{\mu} \partial_{\mu} \rho=m \sigma_{2} \rho^{*}
$$

As a result of the conjugation properties of the $\sigma$-matrices, one can verify that each component of the spinor $\rho$ obeys the Klein-Gordon wave-equation.

In order to display clearly the relationship between eq. (20) and the usual theory of a massive spin 1/2 Dirac fermion, defined by the familiar Lagrangian

$$
L_{D}=-\bar{\Psi} \gamma_{\mu} \partial_{\mu} \Psi-m \bar{\Psi} \Psi
$$

we construct the solutions to eq. (23) in terms of those of eq. (24), which are well known. For this we can use any representation of the Dirac algebra $\gamma_{\mu} \gamma_{\nu}+\gamma_{\nu} \gamma_{\mu}=2 \delta_{\mu \nu}$. To develop the weak interaction theory, however, it is convenient to use the chiral representation, in which $\gamma_{5}$ is diagonal,

$$
\gamma_{i}=\left(\begin{array}{cc}
0 & -i \sigma_{i} \\
i \sigma_{i} & 0
\end{array}\right) \quad \gamma_{4}=\left(\begin{array}{cc}
0 & I \\
I & 0
\end{array}\right) \quad \gamma_{5}=\left(\begin{array}{cc}
I & 0 \\
0 & -I
\end{array}\right)
$$

In this representation the charge conjugation matrix $C$ obeying

$$
\begin{aligned}
C^{T} & =-C \\
C^{\dagger} & =C^{-1} \\
C^{-1} \gamma_{\mu} C & =-\gamma_{\mu}^{T}
\end{aligned}
$$

is simply given by

$$
C=\left(\begin{array}{cc}
-\sigma_{2} & 0 \\
0 & \sigma_{2}
\end{array}\right)
$$

A Dirac spinor may then be written as

$$
\Psi_{D}=\left(\begin{array}{c}
\chi \\
\sigma_{2} \phi^{*}
\end{array}\right)
$$

so that the corresponding charge-conjugate spinor $\Psi_{D}^{c}=C \bar{\Psi}_{D}^{T}$ is the same as $\Psi_{D}$ but exchanging $\phi$ and $\chi$, i.e.,

$$
\Psi_{D}^{c}=\left(\begin{array}{c}
\phi \\
\sigma_{2} \chi^{*}
\end{array}\right)
$$


A 4-component spinor is said to be Majorana or self-conjugate if $\Psi=C \bar{\Psi}^{T}$ which amounts, from eq. (28) and eq. (29) to setting $\chi=\phi$. Using eq. (28) we can rewrite eq. (24) as follows

$$
L_{D}=-i \sum_{\alpha=1}^{2} \rho_{\alpha}^{\dagger} \sigma_{\mu} \partial_{\mu} \rho_{\alpha}-\frac{m}{2} \sum_{\alpha=1}^{2} \rho_{\alpha}^{T} \sigma_{2} \rho_{\alpha}+H . C .
$$

where

$$
\begin{aligned}
& \chi=\frac{1}{\sqrt{2}}\left(\rho_{2}+i \rho_{1}\right) \\
& \phi=\frac{1}{\sqrt{2}}\left(\rho_{2}-i \rho_{1}\right)
\end{aligned}
$$

are the left handed components of $\Psi_{D}$ and of the charge-conjugate field $\Psi_{D}^{c}$, respectively. This way the Dirac fermion is shown to be equivalent to two Majorana fermions of equal mass. As a result of this mass degeneracy, the theory described by eq. (24) is invariant under a continuous rotation symmetry between $\rho_{1}$ and $\rho_{2}$

$$
\begin{array}{r}
\rho_{1} \rightarrow \cos \theta \rho_{1}+\sin \theta \rho_{2} \\
\rho_{2} \rightarrow-\sin \theta \rho_{1}+\cos \theta \rho_{2}
\end{array}
$$

This continuous symmetry is what corresponds to the phase symmetry $\Psi_{D} \rightarrow e^{i \alpha} \Psi_{D}$ associated to fermion number conservation in the Dirac theory.

The mass term in eq. (20) vanishes unless $\rho$ and $\rho^{*}$ are anti-commuting, so we consider the Majorana fermion, right from the start, as a second quantized field. In order to obtain solutions of eq. (23) we start from the usual Fourier expansion for the Dirac spinor,

$$
\Psi_{D}=(2 \pi)^{-3 / 2} \int d^{3} k \sum_{r=1}^{2}\left(\frac{m}{E}\right)^{1 / 2}\left[e^{i k \cdot x} a_{r}(k) u_{r}(k)+e^{-i k \cdot x} b_{r}^{\dagger}(k) v_{r}(k)\right]
$$

where $u=C \bar{v}^{T}$ and $E(k)=\left(\vec{k}^{2}+m^{2}\right)^{1 / 2}$ is the mass-shell condition. From eq. (31) we then derive the corresponding expansion for one of our 2-component Majorana spinors. For example, for $\Psi_{M}=\rho_{2}$ the expansion is

$$
\Psi_{M}=(2 \pi)^{-3 / 2} \int d^{3} k \sum_{r=1}^{2}\left(\frac{m}{E}\right)^{1 / 2}\left[e^{i k . x} A_{r}(k) u_{L r}(k)+e^{-i k \cdot x} A_{r}^{\dagger}(k) v_{L r}(k)\right]
$$

with a similar expression for $\rho_{1}$. Here $L$ denotes left-handed chiral projection, and the operators $A$ are defined are $A=(a+b) / \sqrt{2}$, for each value of $r$ and $k$, from where it follows that they obey canonical anti-commutation rules. Note that eq. (34) expresses the massive Majorana field operator in terms of the chiral projections of the ordinary massive Dirac wave functions $u$ and $v$. Note also that the same creation and annihilation operators appear in eq. (34), showing explicitly that there are only half the number of degrees of freedom in the Majorana field. This gives a consistent Fock-space particle interpretation of the Majorana theory. For example, one 
may look at quantum observables such as the energy momentum, written in terms of the second quantized Majorana field operator given above. One finds in this case that

$$
P_{\mu}=\int d^{3} k \sum_{r=1}^{2} k_{\mu} A_{r}^{\dagger}(k) A_{r}(k),
$$

apart from the zero point energy.

Another important concept of the Majorana theory are the propagators that follow from eq. (20). Lorentz invariance implies that there are 2 different kinds of propagators, i.e.

$$
\begin{array}{r}
<0\left|\rho(x) \rho^{*}(y)\right| 0>=i \sigma_{\mu} \partial_{\mu} \Delta_{F}(x-y ; m) \\
<0|\rho(x) \rho(y)| 0>=m \sigma_{2} \Delta_{F}(x-y ; m)
\end{array}
$$

where $\Delta_{F}(x-y ; m)$ is the usual Feynman function. The first one is the "normal" propagator that intervenes in total lepton number conserving $(|\Delta L|=0)$ processes, while the one in eq. (37) describes the virtual propagation of Majorana neutrinos in $|\Delta L|=2$ processes such as neutrino-less double-beta decay.

It is instructive to consider the massless limit of the Majorana theory. For this purpose we define helicity eigenstate wave-functions by

$$
\begin{aligned}
\vec{\sigma} \cdot \vec{k} u_{L}^{ \pm}(k) & = \pm|\vec{k}| u_{L}^{ \pm}(k) \\
\vec{\sigma} \cdot \vec{k} v_{L}^{ \pm}(k) & =\mp|\vec{k}| v_{L}^{ \pm}(k)
\end{aligned}
$$

The old 2-component massless neutrino theory is recovered from this by noting that, out of the 4 linearly independent wave functions $u_{L}^{ \pm}(k)$ and $v_{L}^{ \pm}(k)$, only two survive as the mass approaches zero, namely, $u_{L}^{-}(k)$ and $v_{L}^{+}(k)$ 13.

In summary, eq. (20) represents a perfectly consistent Lorentz invariant quantum field theory [6]. It can easily be generalised for a system of an arbitrary number of Majorana neutrinos. In this case the most general Lagrangian allowed by Lorentz invariance is of the type

$$
L_{M}=-i \sum_{\alpha=1}^{n} \rho_{\alpha}^{\dagger} \sigma_{\mu} \partial_{\mu} \rho_{\alpha}-\frac{1}{2} \sum_{\alpha, \beta=1}^{n} M_{\alpha \beta} \rho_{\alpha}^{T} \sigma_{2} \rho_{\beta}+\text { H.C. }
$$

where the sum runs over $\alpha$ and $\beta$. By Fermi statistics the mass coefficients $M_{\alpha \beta}$ must form a symmetric matrix, in general complex. This matrix can always be diagonalized by a complex $n \times n$ unitary matrix $U[6]$

$$
M_{\text {diag }}=U^{T} M U
$$

When $M$ is taken to be real (CP conserving) its diagonalizing matrix $U$ may be chosen to be orthogonal and, in general, the mass eigenvalues can have different signs. These may be assembled as a signature matrix

$$
\eta=\operatorname{diag}(+,+, \ldots,-,-, . .)
$$


In eq. (30) and eq. (31) I chose to get rid of these signs by introducing appropriate factors of $i$ in the wave functions. This is perfectly consistent, as long as one only has the free theory given by eq. (20). In the presence of interactions, such as the charged currents of a realistic weak interaction gauge theory, one must bear in mind that these signs are physical, and theories characterized by different signature matrices differ in an essential way. For example, there are two inequivalent models containing 2 Majorana neutrinos: one characterised by $\eta=\operatorname{diag}(+,+)$ and another by $\eta=\operatorname{diag}(+,-)$. A Dirac neutrino belongs to the second class. For example, the condition for CP invariance is different for these two cases. These signs play an important role in the discussion of neutrino-less double beta decay [14].

It should be apparent from the above analysis that there is no reason, in general, to expect a conserved fermion number symmetry to arise in a gauge theory where the basic building blocks are 2-component massive electrically neutral fermions, such as neutrinos or the supersymmetric inos. Unfortunately, the Majorana or Dirac nature of neutrinos can only be distinguished to the extent that neutrinos are massive particles. So far this has proven to be a very severe limiting factor.

\subsection{Neutrinos in The Standard Model and Beyond}

In the Standard Model the only fermions which are electrically neutral, without right-handed partners, and apparently massless are the neutrinos [15]. It is rather mysterious that they seem to be so special. The presence of right handed neutrinos would make the particle spectrum more symmetric between quarks and leptons. In this case neutrinos could get a Dirac mass like the quarks. The presence of $S U(2) \otimes U(1)$ singlet right-handed neutrinos is required in many unified extensions of the Standard Model, such as SO(10), in order to realize the higher symmetry. However, having no electric charge, such neutrinos can also have a large Majorana mass term $M_{R}$. This term violates total lepton number, or B-L (baryon minus lepton number), a symmetry that plays an important role in these extended gauge models [16. In the presence of the usual Dirac mass term this leads to an effective Majorana mass term for the left-handed neutrinos via the so-called seesaw mechanism [17]. The masses of the light neutrinos are obtained by diagonalizing the following mass matrix

\begin{tabular}{c|cc} 
& $\nu$ & $\nu^{c}$ \\
\hline$\nu$ & 0 & $D$ \\
$\nu^{c}$ & $D^{T}$ & $M_{R}$
\end{tabular}


where $D=h_{D} v / \sqrt{2}$ is the Dirac mass matrix and $M_{R}=M_{R}^{T}$ is the isosinglet Majorana mass. In the seesaw approximation, one finds

$$
M_{L}=-D M_{R}^{-1} D^{T}
$$

This mechanism is able to explain naturally the relative smallness of neutrino masses [17. Although one expects $M_{R}$ to be large, its magnitude heavily depends on the model. Although the seesaw idea was suggested in the context of $\mathrm{SO}(10)$ or left-right symmetric extensions where lepton number is a part of the gauge symmetry [16], it may be directly introduced in the Standard Model and the value of the scale may be as low as the TeV scale. Although very often one hears about seesaw "predictions" for light neutrino masses, they often depend on specific assumptions. In general, one can not make any generic prediction for the light neutrino masses that are generated through the exchange of the heavy right-handed neutrinos. For example, the mass formula in eq. (43) would suggest that the light neutrino masses scale with the up-type quark masses as $m_{\nu_{e}} \propto m_{u}^{2}, m_{\nu_{\mu}} \propto m_{c}^{2}$ and $m_{\nu_{\tau}} \propto m_{t}^{2}$. Unfortunately this is not really true for two reasons: first the existence of an induced triplet VEV and, second, due to the dependence upon the detailed structure not only of the Dirac type entries, but also on the possible texture of the large Majorana mass term [18]. As a result, the seesaw mechanism provides us only with a general scheme, rather than detailed predictions.

Although attractive, the seesaw mechanism is by no means the only way to generate neutrino masses. There is a large diversity of possible schemes to generate neutrino masses, which do not require any new large mass scale. For example, it is possible to start from an extension of the lepton sector of the $S U(2) \otimes U(1)$ theory by adding a set of two 2-component isosinglet neutral fermions, denoted $\nu^{c}{ }_{i}$ and $S_{i}$. In this case there is an exact L symmetry that keeps neutrinos strictly massless, as in the Standard Model. The conservation of total lepton number leads to the following form for the neutral mass matrix

\begin{tabular}{c|ccc} 
& $\nu$ & $\nu^{c}$ & $S$ \\
\hline$\nu$ & 0 & $D$ & 0 \\
$\nu^{c}$ & $D^{T}$ & 0 & $M$ \\
$S$ & 0 & $M^{T}$ & 0
\end{tabular}

This form has also been suggested in various theoretical models [19, including many of the superstring inspired models. In the latter case the zeros of eq. (44) naturally arise due to the absence of Higgs fields to provide the usual Majorana mass terms, needed in the seesaw model [20]. The implications of eq. (44) are interesting on their own right, and the model represents a conceptually simple and phenomenologically rich extension of the Standard Model, which brings in the possibility that a wide range of new phenomena be sizeable. These have to do 
with neutrino mixing, universality, flavour and CP violation in the lepton sector [21, 22, 20], as well as direct effects of associated with the NHL production in $Z$ decays 23. Clearly, one can easily introduce non-zero masses in this model through a $\mu S S$ term that could be proportional to the VEV of a singlet field $\sigma$ [24]. In contrast to the seesaw scheme, the neutrino masses are directly proportional to $\langle\sigma\rangle$, a fact which is very important for the phenomenology of the Higgs boson sector.

There is also a large variety of possible radiative schemes to generate neutrino masses with explicitly broken lepton number, which do not require one to introduce a large mass scale. It is quite possible embed such schemes so as to have the spontaneous violation of the global lepton number symmetry. The scale at which such a symmetry gets broken does not need to be high, as in the original proposal [25], but can be rather low, close to the weak scale [26]. Such models are very attractive and lead to a richer phenomenology, as the extra particles required have masses at scales that could be accessible to present experiments [27, 28].

The physics of neutrino masses and lepton number violation could have important implications not only in astrophysics and cosmology but also in particle physics.

\subsection{Leptonic Charged Currents}

In analogy with the case of quarks, one can give a mass to neutrinos by adding right-handed singlet neutrinos to table 1 , denoted by $\nu_{i}^{c}$, through a new gauge invariant Yukawa interaction,

$$
h_{D i a} \nu_{i}^{c} \ell_{a} \tau_{2} \phi^{*}
$$

This generates a Dirac mass for the neutrinos which preserves the total lepton number. However, unlike the right-handed charged fermions, the right-handed neutrinos, are singlets under the full $S U(2) \otimes U(1)$ gauge group. As a result, the number $m$ of the $\nu_{i}^{c}$ fields, $1 \leq i \leq m$, is completely free and does not need to match the number of left-handed neutrinos [7].

As we have seen above, in gauge theories of massive neutrinos there is in general no reason to have a conserved total lepton number. Indeed, in many gauge theories it is precisely the violation of lepton number that generates the masses for the neutrinos, either as a result of an enlarged Higgs sector or due a seesaw mechanism involving the exchange of some heavy neutral leptons. In this case neutrinos are Majorana particles and their mass term is of the form given in eq. (39).

The form of the leptonic charged current will depend on whether there are isosinglet neutrinos and how many. It will also be quite sensitive to whether neutrinos are of Dirac or Majorana type. 


\section{- Dirac neutrinos}

We first consider the case where the number $m$ of isosinglets is smaller than that of isodoublet neutrinos, $m<n$. In this case $n-m$ neutrinos remain massless. As a result, the structure of lepton mixing is simpler than that in eq. (18) because one can perform arbitrary rotations (not only rephasings) in the degenerate sector of massless neutrinos and this way eliminate unphysical parameters. The simplest model of the $(n, m)$ type, with $m \neq 1$ is one with a conserved total lepton number and contains [0]

$$
n(n-1) / 2-(n-m)(n-m-1) / 2
$$

mixing angles $\theta_{i j}$ and

$$
1+n(m-1)-m(m+1) / 2
$$

$\mathrm{CP}$ violating phases $\phi_{i j}$. For the case of 3 generations and a single right handed neutrino added $(n=3, m=1)$ the mixing matrix can always be brought to the form [7]

$$
K=R_{23} R_{13}=\left(\begin{array}{ccc}
c_{13} & 0 & s_{13} \\
-s_{23} s_{13} & c_{23} & s_{23} c_{13} \\
-s_{13} c_{23} & -s_{23} & c_{13} c_{23}
\end{array}\right)
$$

where $R_{i j}=\omega_{i j}\left(\phi_{i j}=0\right)$ is a real rotation by an angle $\theta_{i j}$ in the $i j$ plane. Eq. (48) shows explicitly how one of the 3 mixing angles has been eliminated, together with the would-be $\mathrm{CP}$ violating phase. When $n=m$ the structure of lepton mixing of Dirac neutrinos is identical with that describing quark mixing, eq. (18), so the same parametrization applies. When the number of isosinglets is larger than that of isodoublets, $m>n$, new conceptual possibilities arise for the physics of leptons. For example, if $m=2 n$, the masslessness of the observed neutrinos may be enforced by imposing the conservation of total lepton number, yet allowing for the remarkable possibility of lepton flavour and CP non-conservation despite neutrinos being strictly massless [21, 22, 23, 29].

\section{- Majorana neutrinos}

In the case of Majorana neutrinos the mass matrix eq. (39) is not invariant under rephasings of the neutrino fields. As a result, there are new phases present which cause CP to be violated in a theory with just two generations of Majorana neutrinos [6, 31]. The charged current of a 2-generation model $(n=2, m=0)$ may be parametrised as

$$
\left(\begin{array}{cc}
c_{12} & e^{i \phi_{12}} s_{12} \\
-e^{-i \phi_{12}} s_{12} & c_{12}
\end{array}\right)
$$

where $\phi_{12}$ is the Majorana phase. For $n$ generations these Majorana phases make up a total of $(n-1)$ additional phases over and above those of eq. (17). They are physical 
parameters, and play a role in $|\Delta L|=2$ processes such as some class of neutrino oscillations [31]. Although conceptually important, the $\mathrm{CP}$ violating effects associated to these phases are too small to be observed, since they are helicity suppressed. An important subtlety arises regarding the conditions for $\mathrm{CP}$ conservation in theories of massive Majorana neutrinos. Unlike the case of Dirac fermions, in the Majorana case the condition for CP invariance is [13]

$$
K^{*}=K \eta
$$

where $\eta=\operatorname{diag}(+,+, \ldots,-,-, .$.$) is the signature matrix describing the relative signs of the$ neutrino mass eigenvalues that follow from eq. (39) obtained by using real diagonalizing matrices [14, 13]. Only for the trivial case where $\eta$ is trivial, CP invariance requires the mixing matrix to be real. For example the value $\phi_{12}=\pi / 2$ (just as $\phi_{12}=0$ ) can be $\mathrm{CP}$ conserving. We now turn to the most general situation where there are $m \neq 0$ twocomponent $S U(2) \otimes U(1)$ singlet leptons (such as RH neutrinos) present in the theory. In this case there is in general no reason to forbid a gauge and Lorentz invariant Majorana mass term of the type

$$
M_{R i j} \nu_{i}^{c} \nu_{j}^{c}
$$

which breaks total lepton number symmetry. As a result, the structure of the weak currents can be substantially more complex. Since the number $m$ is arbitrary, one may consider models with Majorana neutrinos based on any value of $m$ [7]. For $m \leq n, n-m$ neutrinos will remain massless, while $2 m$ neutrinos will acquire Majorana masses. For example, in a model with $n=3$ and $m=1$ one has one light (presumably $\nu_{\tau}$ ) and one heavy Majorana neutrino, in addition to the two massless neutrinos $\left(\nu_{e}\right.$ and $\left.\nu_{\mu}\right)$. In this case clearly there will be less parameters than present in a model with $m=n=3$. The case $m>n$, e.g., $m=2 n$, may also be interesting because it allows for an elegant way to avoid constraints related to neutrino masses, and therefore enhance many of the effects possible in these theories [24, 23, 20, 32]. I will now analyse some of the general features of the associated currents. The first is that the isosinglets, presumably heavy, will now mix with the ordinary isodoublet neutrinos in the charged current weak interaction. As a result, the mixing matrix describing the charged leptonic weak interaction is a rectangular matrix $K$ [6], which may be decomposed as

$$
K=\left(K_{L}, K_{H}\right)
$$

where $K_{L}$ is an $n \times n$ matrix and $K_{H}$ is an $n \times m$ matrix. The charged weak interactions of the light (mass-eigenstate) neutrinos are effectively described by a mixing matrix $K_{L}$ which is non-unitary. An explicit parametrization of the weak charged current mixing matrix $K$ that covers the most general situation present in these $(n, m)$ models has also been given in ref. [6]. It involves in general

$$
n(n+2 m-1) / 2
$$


mixing angles $\theta_{i j}$ and

$$
n(n+2 m-1) / 2
$$

$\mathrm{CP}$ violating phases $\phi_{i j}$. This number far exceeds the corresponding number of parameters describing the charged current weak interaction of quarks eq. (16) and eq. (17). The reasons are that, since neutrinos are Majorana particles, their mass terms are not invariant under rephasings [6]. As a result, there are less phases that can be eliminated by field redefinitions. Their possible role in neutrino oscillations was studied in ref. [31. In addition, the isodoublet neutrinos in general mix with the isosinglets, so CP may also be violated in this mixing, even in the case where the physical light neutrinos are massless 22 .

\subsection{Leptonic Neutral Current}

The neutral current couplings of mass-eigenstate neutrinos are diagonal, just as in the case of charged leptons and quarks, in theories where there are no isosinglet neutrinos , i.e., $m=$ 0. This occurs irrespective of whether the mass-eigenstate neutrinos are Dirac or Majorana particles.

An important feature arises in any theory based on $S U(2) \otimes U(1)$, where isosinglet and isodoublet lepton mass terms coexist, regardless of $L$ number conservation. In this case the gauge currents mix neutrinos with isosinglet leptons. As a result, in these theories there can be non-diagonal couplings of the $Z$ to the mass-eigenstate neutrinos, even at the tree level [6]. The neutral current may be expressed in the following general form

$$
P=K^{\dagger} K
$$

where the matrix $\mathrm{P}$ is a projective hermitian matrix $P^{2}=P=P^{\dagger}$, directly determined in terms of eq. (52) as

$$
P=\left(\begin{array}{cc}
K_{L}^{\dagger} K_{L} & K_{L}^{\dagger} K_{H} \\
K_{H}^{\dagger} K_{L} & K_{H}^{\dagger} K_{H}
\end{array}\right)
$$

This matrix determines the neutral current couplings of all mass-eigenstate neutral leptons (denoted $N_{L \alpha}$ ), both the light neutrinos as well as the NHLS, as follows

$$
\frac{i g^{\prime}}{2 \sin \theta_{W}} Z_{\mu} \sum_{\alpha \beta} \bar{N}_{L \alpha} \gamma_{\mu} P_{\alpha \beta} N_{L \beta}
$$

In summary we see that while the structure of the leptonic weak interaction is completely trivial within the Standard Model, when compared to the quark weak interactions, it may be much more complex in theories beyond the Standard Model. These could give rise to a rich body of phenomena which may or may not be directly related to the magnitude of the neutrino masses, as discussed below. 


\subsection{Constraints on neutrino-NHL mixing}

There are important constraints on the leptonic charged current matrix elements and, in particular, on the allowed magnitude of neutrino-NHL mixing. For example, from eq. (10) and eq. (52) we see that the coupling of a given light neutrino to the corresponding charged lepton is decreased by a certain factor. This observed universality of weak interactions constrains the relative values of these decreases and limits them to be small [29]. In the low mass range the existence of these new leptons could show up explicitly in low energy weak decay processes, if such neutrinos can be kinematically produced. Heavier masses above the few GeV range can be ideally searched for at LEP experiments at the $\mathrm{Z}$ pole. Constraints on the strength of the $K_{H}$ mixing matrix elements follow therefore from low energy weak decay measurements as well as from LEP experiments. They are shown in Fig. (1), from ref. [30].

\subsection{Phenomenological Implications.}

One of the simplest and natural extensions of the lepton sector is the addition of neutral isosinglet heavy leptons, such as right-handed neutrinos. Their existence is required, for example, in left-right symmetric models [16] and leads, via the seesaw mechanism, to non-vanishing neutrino masses. However, they may be added simply at the $S U(2) \otimes U(1)$ level and, since they are $\mathrm{SU}(2)_{L}$ singlets, their number is totally free, as they carry no anomalies [6]. There is a variety of novel phenomena whose existence would be associated to these extensions of the lepton sector [15. After mass matrix diagonalization one finds that these leptons will couple in the charged and neutral weak currents. For example, the matrix $P$ in eq. (57) contains couplings connecting light to heavy neutrinos [6]. Thus, if their mass is below that of the $Z$, the heavy ones will be singly produced in $Z$ decays, 23]

$$
Z \rightarrow N_{\tau}+\nu_{\tau}
$$

Subsequent NHL decays would then give rise to large missing momentum events, called zenevents. The attainable rates for such processes can be quite large [23], well within the sensitivities of the LEP experiments [23]. Dedicated searches for acoplanar jets and lepton pairs from $Z$ decays have provided stringent constraints on NHL couplings to the $Z$, plotted below [30]

One sees that the recent DELPHI constraints supersede by far the low energy constraints following, e.g. from weak universality.

Let us now turn to the case when the isosinglet neutral heavy leptons are heavier than the $Z$. Even in this case they can produce interesting virtual effects, completely calculable in these models, in terms of the NHL masses and electroweak charged and neutral current couplings.

\footnotetext{
${ }^{c}$ There have been also inconclusive hints reported by ALEPH [33].
} 


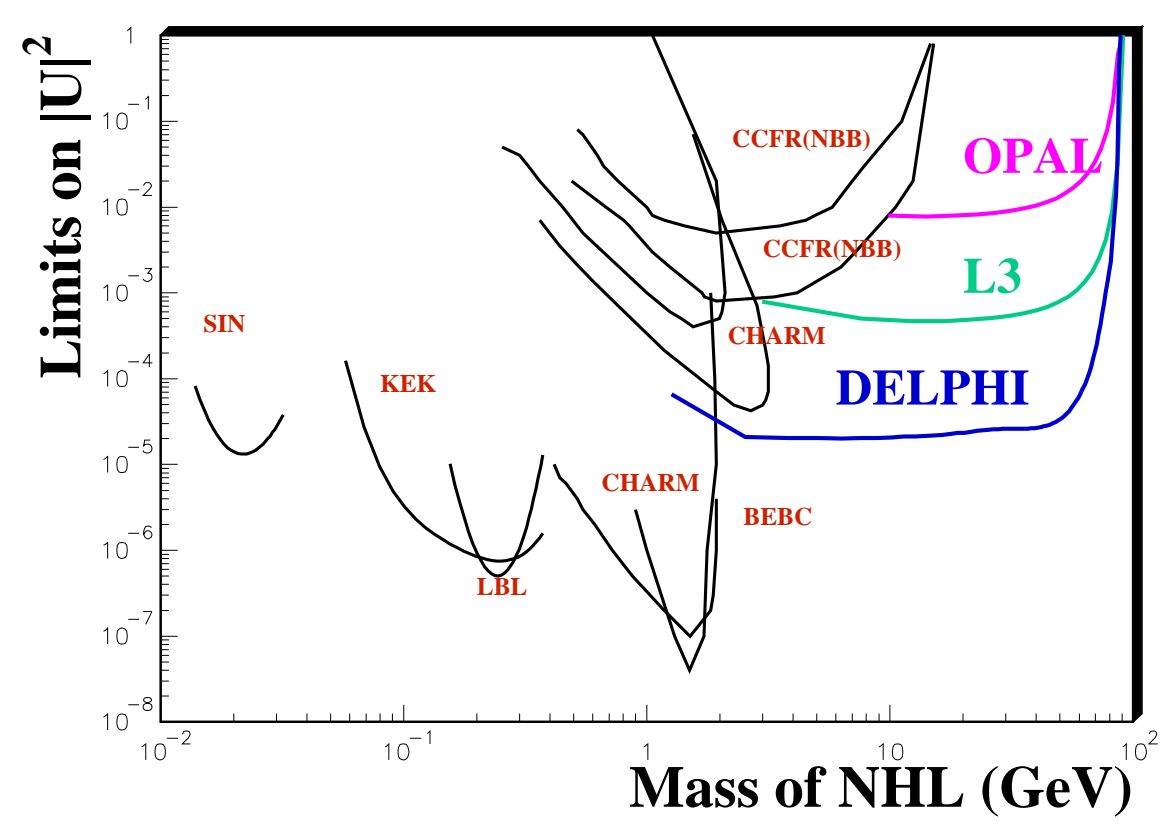

Figure 1: Limits on NHL mass and couplings.

Table 2: Allowed lepton-flavour-violating $\tau$ decay branching ratios.

\begin{tabular}{|c|c|}
\hline channel & strength \\
\hline$\tau \rightarrow e \gamma, \mu \gamma$ & $\lesssim 10^{-6}$ \\
$\tau \rightarrow e \pi^{0}, \mu \pi^{0}$ & $\lesssim 10^{-6}$ \\
$\tau \rightarrow e \eta^{0}, \mu \eta^{0}$ & $\lesssim 10^{-6}-10^{-7}$ \\
$\tau \rightarrow 3 e, 3 \mu, \mu \mu e$, etc. & $\lesssim 10^{-6}-10^{-7}$ \\
\hline
\end{tabular}

This way they can mediate lepton flavour violating (LFV) decays which are exactly forbidden in the Standard Model, thus a clear signature of physics beyond the Standard Model. In the simplest models of seesaw type where the NHLS are Majorana type these decays are expected to be small, due to limits on the light neutrino masses. However, in other variant models with Dirac NHLS [20] this suppression is not present [21, 22] and LFV rates are restricted only by present constraints on weak universality violation. These allow for sizeable decay branching ratios, close to present experimental limits [34 and within the sensitivities of the planned tau and B factories [35]. The situation is summarised in Tables 2 and 3. The dependence of the attainable LFV $\tau$ and Z decay branching ratios upon the NHL mass is illustrated in ref. 36]. The study of rare $Z$ decays nicely complements what can be learned from LFV muon and tau decays. The stringent limits on $\mu \rightarrow e \gamma$ preclude sizeable branching ratios for the corresponding 
Table 3: Allowed lepton-flavour-violating $Z$ decay branching ratios.

\begin{tabular}{|l|l|}
\hline channel & strength \\
\hline$Z \rightarrow e \tau$ & $\lesssim 10^{-6}$ \\
$Z \rightarrow \mu \tau$ & $\lesssim 10^{-7}$ \\
\hline
\end{tabular}

Table 4: Delphi limits on lepton-flavour-violating $\tau$ decays.

\begin{tabular}{|c|c|}
\hline channel & $90 \%$ C.L. limit \\
\hline$\tau \rightarrow e \gamma$ & $1.1 \times 10^{-4}$ \\
$\tau \rightarrow \mu \gamma$ & $6.2 \times 10^{-5}$ \\
\hline
\end{tabular}

process $Z \rightarrow e \mu$. However the decays $Z \rightarrow e \tau$ and $Z \rightarrow \mu \tau$ can occur at the $\mathcal{O}\left(10^{-6}\right)$ level, as illustrated in table 3. Similar statements can be made also for the $\mathrm{CP}$ violating $\mathrm{Z}$ decay asymmetries in these LFV processes [22]. However, under realistic assumptions, it is unlikely that one will be able to see these decays at LEP without a high luminosity option [32]. In any case there have been dedicated experimental searches which have set good limits on LFV Z and $\tau$ decays at LEP [37. This is illustrated in tables 4 and 5 .

Finally we note that there can also be large rates for lepton flavour violating decays in models with radiative mass generation [27, 28]. A very interesting observation is that LFV phenomena

Table 5: Opal limits on lepton-flavour-violating Z decays.

\begin{tabular}{|c|c|}
\hline channel & $95 \%$ C.L. limit \\
\hline$Z \rightarrow e \mu$ & $1.7 \times 10^{-6}$ \\
$Z \rightarrow e \tau$ & $9.8 \times 10^{-6}$ \\
$Z \rightarrow \mu \tau$ & $17 \times 10^{-6}$ \\
\hline
\end{tabular}




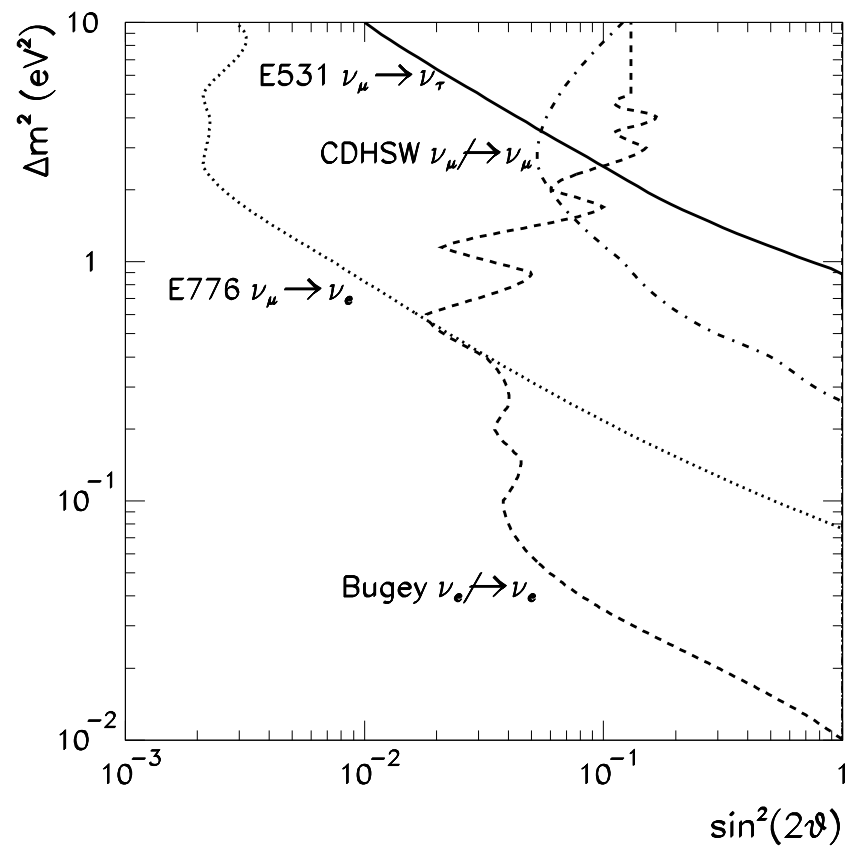

Figure 2: Status of neutrino oscillation parameters.

are expected in supersymmetric unified models [38]. This has been discussed in many papers [39] and the predictions have now been recently reanalysed in view of the large top quark mass in ref. [40]. The expected decay rates may lie within the present experimental sensitivities and the situation should improve at PSI or at the proposed tau-charm factories.

\subsection{Laboratory Limits on Neutrino Masses}

- Of all existing neutrino mass limits the most model-independent are the laboratory limits that follow purely from kinematics. These can be summarised as [5]

$$
m_{\nu_{e}} \lesssim 5 \mathrm{eV}, \quad m_{\nu_{\mu}} \lesssim 250 \mathrm{KeV}, \quad m_{\nu_{\tau}} \lesssim 23 \mathrm{MeV}
$$

The limit on the $\nu_{e}$ mass follows from tritium beta decay studies [41], while that on the $\nu_{\tau}$ mass has recently been reported by the ALEPH experiment at CERN [42]. This limit may be substantially improved at a future tau factory [43].

- Neutrino oscillations have been searched in both accelerator and reactor experiments [44]. So far no effect has been conclusively demonstrated, in any of the channels. As a result, limits have been set on the relevant mixings and mass differences. The $90 \%$ confidence level (C.L.) exclusion contours of neutrino oscillation parameters in the 2flavour approximation are given in Fig. (22), taken from ref. [45]. As can be seen, the best limit is for $\nu_{\mu} \rightarrow \nu_{e}$ oscillations. Recently there has been a limit on $\nu_{e} \rightarrow$ $\nu_{\mu}$ oscillations using data from the LSND experiment at Los Alamos 46]. Improvements for the $\nu_{\mu} \rightarrow \nu_{\tau}$ channel are expected soon from the ongoing CHORUS and NOMAD 


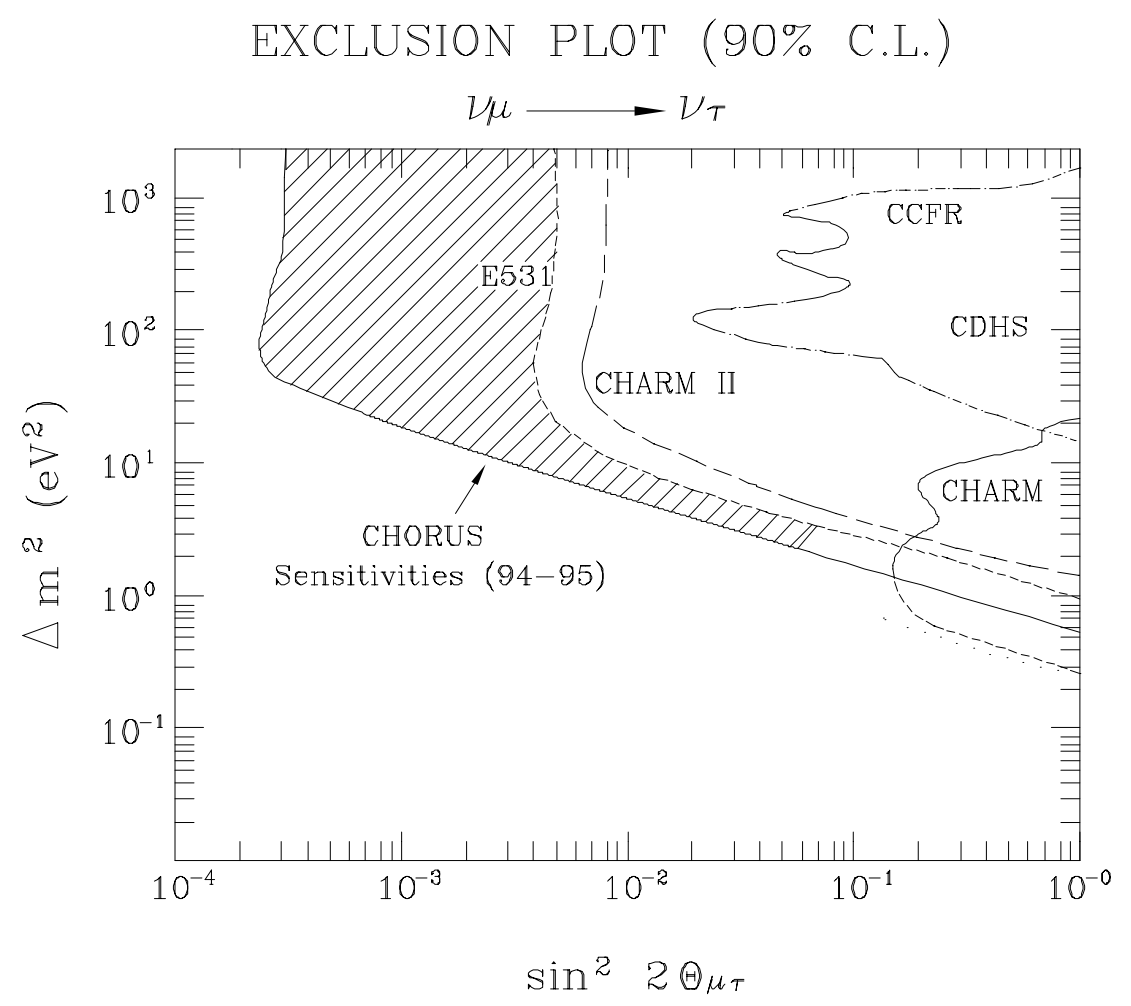

Figure 3: Sensitivity of Chorus experiment.

experiments at CERN, with a similar proposal at Fermilab [47]. For illustration, we give in Fig. (3) the region of sensitivity of Chorus experiment. Finally there are future prospects to probe neutrino oscillation parameters with much better sensitivity at long baseline experiments using CERN, Fermilab and KEK neutrino beams in conjunction with Gran Sasso, Soudan and SuperKamiokande underground installations, respectively. There are also long baseline reactor experiments such as Chooz and San Onofre, with very good sensitivity to neutrino oscillation parameters.

- Another important limit follows from the non-observation of neutrino-less double beta decay - $\beta \beta_{0 \nu}$ - i.e. the process by which an $(A, Z-2)$ nucleus decays to $(A, Z)+2 e^{-}$. This process would arise from the virtual exchange of a Majorana neutrino from an ordinary double beta decay process. Unlike the latter, the neutrino-less process is lepton number violating and its existence would signal the Majorana nature of neutrinos. Because of the phase space advantage, this process is a very sensitive tool to probe into the nature of neutrinos. In fact, as shown in ref. [48], a non-vanishing $\beta \beta_{0 \nu}$ decay rate requires neutrinos to be Majorana particles, irrespective of which mechanism induces it. This establishes a very deep connection which, in some special models, may be translated into a lower limit on the neutrino masses. The negative searches for $\beta \beta_{0 \nu}$ in ${ }^{76} \mathrm{Ge}$ and other nuclei leads to a limit of about two $\mathrm{eV}$ 49 on the weighted average neutrino mass parameter $\langle m\rangle$

$$
\langle m\rangle \lesssim 1-2 \mathrm{eV}
$$



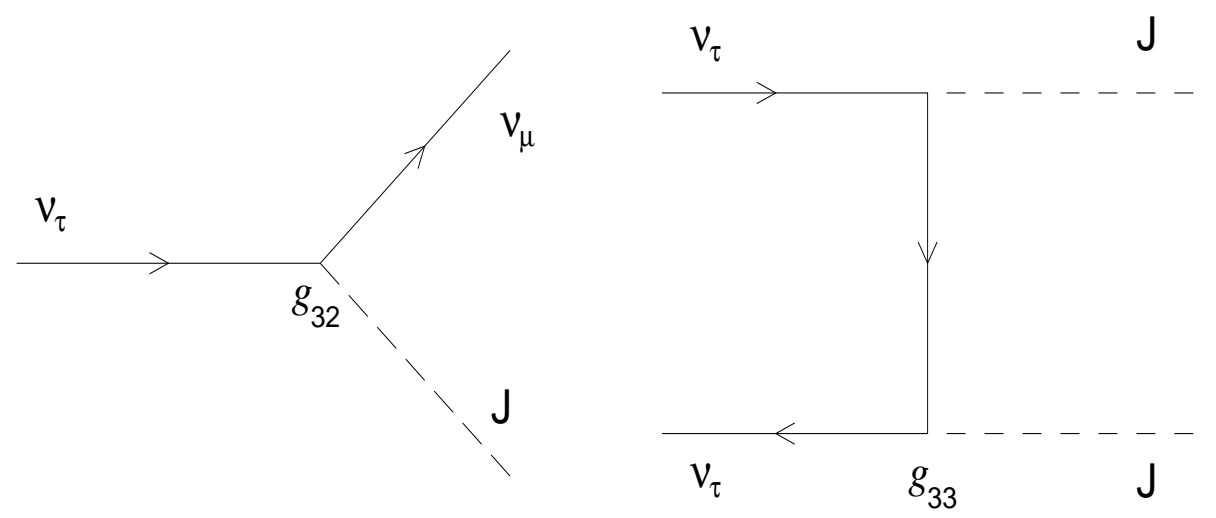

Figure 4: Majorana tau neutrino decays and annihilations to Majorons.

depending to some extent on the relevant nuclear matrix elements characterising this process [50]. Improved sensitivity is expected from the upcoming enriched germanium experiments. Although rather stringent, this limit in eq. (60) may allow relatively large neutrino masses, as there may be strong cancellations between the contributions of different neutrino types. This happens automatically in the case of a Dirac neutrino as a result of the lepton number symmetry [51].

\subsection{The Cosmological Density Limit}

In addition to laboratory limits, there is a cosmological bound that follows from avoiding the overabundance of relic neutrinos [52]

$$
\sum_{i} m_{\nu_{i}} \lesssim 92 \Omega_{\nu} h^{2} \mathrm{eV},
$$

where $\Omega_{\nu} h^{2} \leq 1$ and the sum runs over all isodoublet neutrino species with mass less than $\mathcal{O}$ (1) MeV. Here $\Omega_{\nu}=\rho_{\nu} / \rho_{c}$, where $\rho_{\nu}$ is the neutrino contribution to the total density and $\rho_{c}$ is the critical density. The factor $h^{2}$ measures the uncertainty in the determination of the present value of the Hubble parameter, $0.4 \leq h \leq 1$. The factor $\Omega_{\nu} h^{2}$ is known to be smaller than 1.

For the $\nu_{\mu}$ and $\nu_{\tau}$ this bound is much more stringent than the corresponding laboratory limits in (59).

Recently there has been a lot of work on the possibility of an MeV tau neutrino [53, 54]. Such range seems to be an interesting one from the point of view of structure formation [53, 54]. Moreover, it is theoretically viable as the constraint in eq. (61) holds only if neutrinos are stable on the relevant cosmological time scales. As shown in Fig. (四), in models with spontaneous violation of total lepton number 25] neutrinos may decay into a lighter neutrino plus a Majoron, for example [15],

$$
\nu^{\prime} \rightarrow \nu+J .
$$


or have sizeable annihilations (there is also a crossed diagram)

$$
\nu^{\prime} \nu^{\prime} \rightarrow J+J
$$

couplings The possible existence of fast decay and/or annihilation channels could eliminate relic neutrinos and therefore allow them to be heavier than eq. (61). The cosmological density constraint on neutrino decay lifetime (for neutrinos lighter than an $\mathrm{MeV}$ or so) may be written as

$$
\tau_{\nu^{\prime}} \lesssim 1.5 \times 10^{7}\left(\mathrm{KeV} / m_{\nu^{\prime}}\right)^{2} \mathrm{yr}
$$

and follows from demanding an adequate red-shift of the heavy neutrino decay products. For neutrinos heavier than $\sim 1 \mathrm{MeV}$, such as possible for the case of $\nu_{\tau}$, the cosmological limit on the lifetime is less stringent than given in eq. (64).

As we already mentioned the possible existence of non-standard interactions of neutrinos due to their couplings to the Majoron brings in the possibility of fast invisible neutrino decays with Majoron emission [15]. These 2-body decays can be much faster than the visible decays, such as radiative decays of the type $\nu^{\prime} \rightarrow \nu+\gamma$. As a result the Majoron decays are almost unconstrained by astrophysics and cosmology. For a more detailed discussion see ref. [52].

A general method to determine the Majoron emission decay rates of neutrinos was first given in ref. [55]. The resulting decay rates are rather subtle [55] and model dependent and will not be discussed here. The reader may consult ref. [56, 24, 15]. The conclusion is that there are many ways to make neutrinos sufficiently short-lived that all mass values consistent with laboratory experiments are cosmologically acceptable. For neutrino decay lifetime estimates see ref. [15, 56, 24, 57].

\subsection{The Cosmological Nucleosynthesis Limit}

Recently there has been extensive discussion about cosmological nucleosynthesis [58, 59]. The observation of the primordial light element abundances can be used to place important constraints on neutrino properties, such as masses, decay lifetimes and annihilation cross sections. Here we focus on the case of the tau neutrino since, from (59), it is the only neutrino that could have mass in the relevant $\mathrm{MeV}$ region. If massive $\nu_{\tau}$ 's are stable during nucleosynthesis $\left(\nu_{\tau}\right.$ lifetime longer than $\sim 100 \mathrm{sec}$ ), one can constrain their contribution to the total energy density from the observed amount of primordial helium. This bound can be expressed through an effective number of massless neutrino species $\left(N_{\nu}\right)$. Using $N_{\nu}<3.4-3.6$, the following range of $\nu_{\tau}$ mass has been ruled out 60, 61]

$$
0.5 \mathrm{MeV}<m_{\nu_{\tau}}<35 \mathrm{MeV}
$$




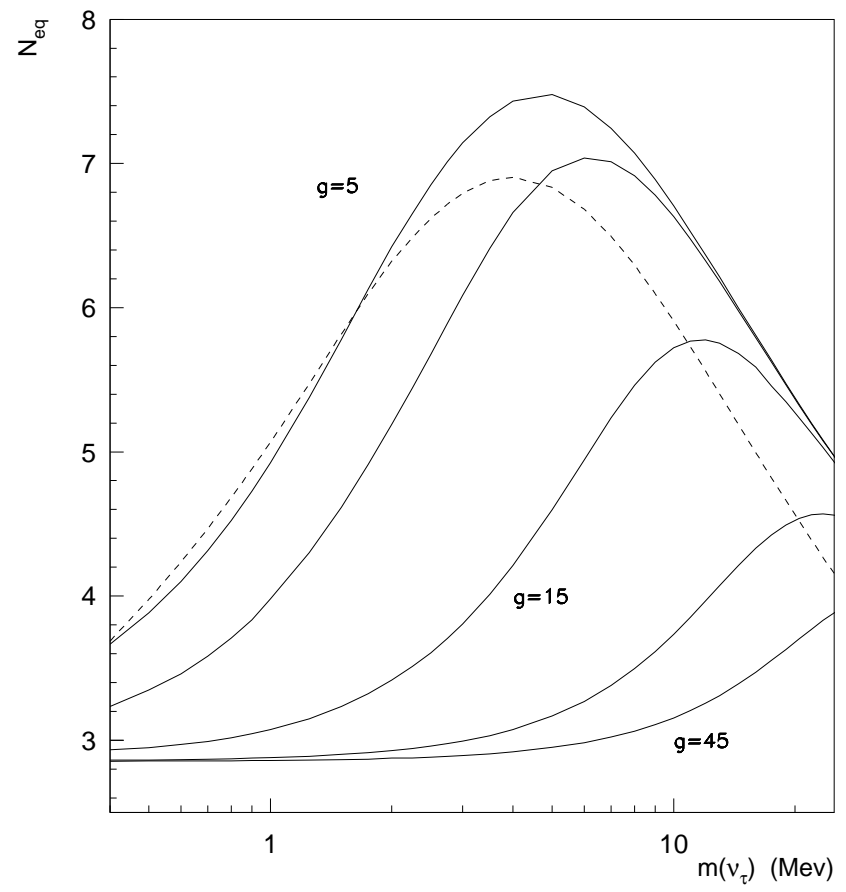

Figure 5: Effective number of massless neutrinos equivalent to the contribution of massive neutrinos.

If the nucleosynthesis limit is taken less stringent the limit loosens somewhat. However it has recently been argued that non-equilibrium effects from the light neutrinos arising from the annihilations of the heavy $\nu_{\tau}$ 's make the constraint stronger and forbids all $\nu_{\tau}$ masses on the few $\mathrm{MeV}$ range.

One can show that if the $\nu_{\tau}$ is unstable during nucleosynthesis 62 the bound on its mass is substantially weakened, depending on the assumed neutrino lifetime.

Even more drastic is the effect of neutrino annihilations [63]. The results are shown in Fig. (5). The solid line gives the effective number of massless neutrinos equivalent to the contribution of the massive $\nu_{\tau}$ for different $g$ values expressed in units $10^{-5}$, while the dashed line corresponds to the Standard Model case when $g=0$ and no Majorons are present. One sees that no $\nu_{\tau}$ masses below $23 \mathrm{MeV}$ can be ruled out as long as the coupling between $\nu_{\tau}$ 's and $J$ 's exceeds a few times $10^{-4}$ or so. Such values are reasonable for many Majoron models [64. For more details see ref. 63. In short one sees that the constraints on the mass of a Majorana $\nu_{\tau}$ from primordial nucleosynthesis can be substantially relaxed if annihilations $\nu_{\tau} \bar{\nu}_{\tau} \leftrightarrow J J$ are present. As a result of the considerations in section 2 one concludes that it is worthwhile to continue the efforts to improve present laboratory neutrino mass limits in the laboratory. One method sensitive to large masses is to search for distortions in the energy spectra of leptons coming from $\pi, K$ weak decays such as $\pi K \rightarrow e \nu, \pi, K \rightarrow \mu \nu$, as well as kinks in nuclear $\beta$ decays. 


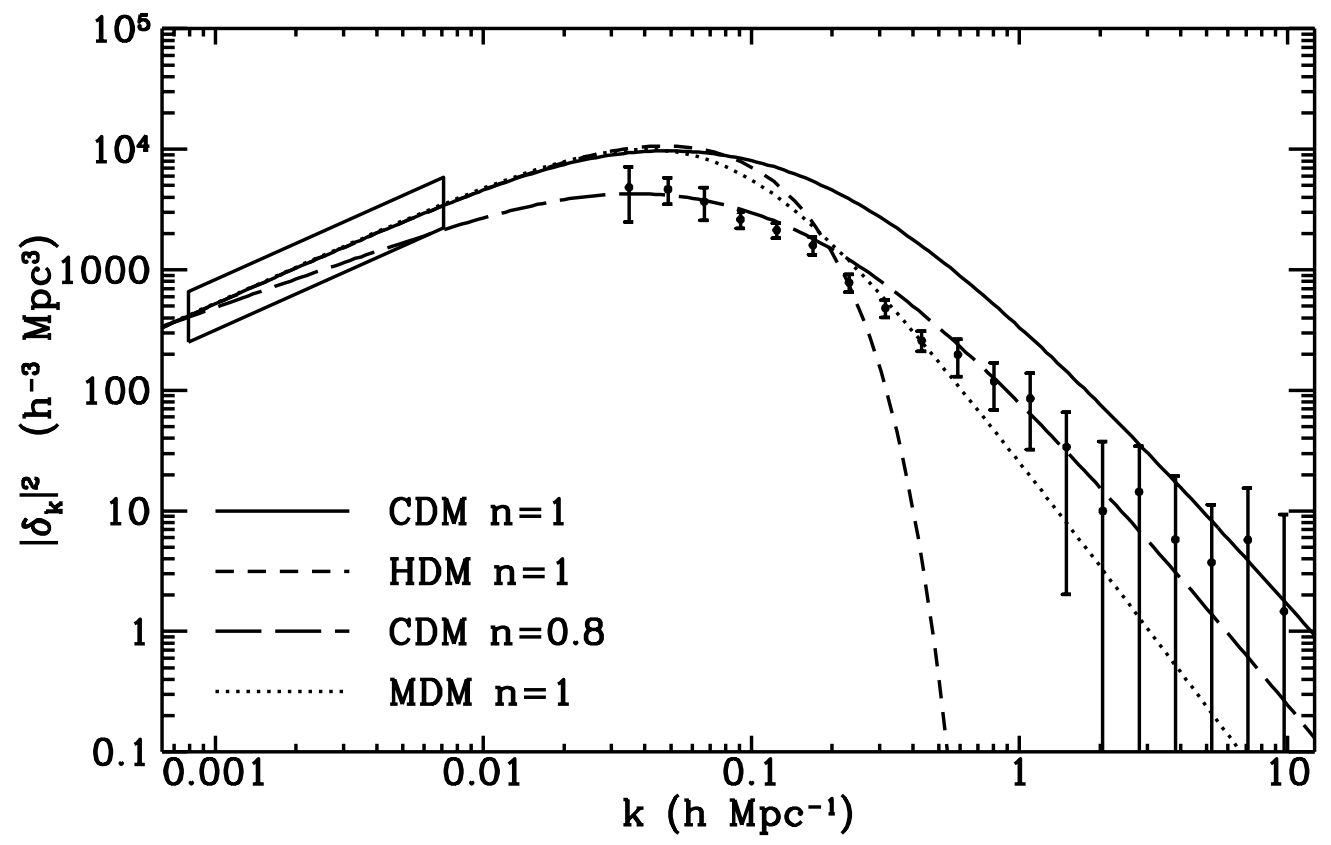

Figure 6: Power spectrum of density perturbations

\subsection{Hints for Neutrino Masses}

The only hint for neutrino masses following from accelerator experiments is the controversial claim for neutrino oscillations reported by the LSND experiment. I will not describe it here, but the reader is encouraged to read the original paper, now published [65].

Thus all positive indications in favour of nonzero neutrino masses follow from astrophysics and cosmology. We now turn to these.

\subsubsection{Dark Matter}

With the COBE detection of fluctuations in the Cosmic Microwave Background (CMB) radiation by the COBE satellite [66] it is now possible to accurately normalize fluctuations on the largest observable scales [67]. By combining these observations on large scales performed with cluster-cluster correlation data e.g. from IRAS [68] one finds that it is not possible to fit well the data on all scales within the framework of the popular cold dark matter (CDM) model. The situation is illustrated in Fig. (6). This figure shows the measured power spectrum of density perturbations and how it compares with the predictions of various models of structure formation. A good fit is obtained for an otherwise ad hoc Mixed Dark Matter (MDM) universe, consisting of about $75 \%$ CDM with about $25 \%$ hot dark matter (HDM), and a very small amount in baryons [67] and with the standard Harrison-Zeldovich $n=1$ spectrum predicted by inflation.

One way to make up for the hot dark matter component is through a massive neutrino in the 
few eV mass range. It has been argued that this could be the tau neutrino, in which case one might expect the existence of $\nu_{e} \rightarrow \nu_{\tau}$ or $\nu_{\mu} \rightarrow \nu_{\tau}$ oscillations. These are now being searched by the CHORUS and NOMAD experiments at CERN. These is also a similar proposal at Fermilab [47.

\subsubsection{Solar Neutrino Experiments}

So far the averaged data collected by the chlorine [69], Kamiokande [70], as well as by the low-energy data on pp neutrinos from the GALLEX and SAGE experiments [71, 72] still pose a persisting puzzle. The most recent data can be summarised as:

$$
R_{C l}^{e x p}=(2.55 \pm 0.25) \mathrm{SNU}, \quad R_{G a}^{e x p}=(74 \pm 8) \mathrm{SNU}, \quad R_{K a}^{e x p}=(0.44 \pm 0.06) R_{K a}^{B P 95}
$$

where $R_{K a}^{B P 95}$ is the Bahcall-Pinsonneault (BP95) SSM prediction of ref. [73]. For the gallium result we have taken the average of the GALLEX $R_{G a}^{e x p}=(77 \pm 8 \pm 5) \mathrm{SNU}$ [71] and the SAGE measurements $R_{G a}^{e x p}=(69 \pm 11 \pm 6) \mathrm{SNU}[72]$.

Comparing the data of gallium experiments with the Kamiokande data one sees the need for a reduction of the ${ }^{7}$ Be flux relative to Standard Solar Model [73] expectations. Inclusion of the Homestake data only aggravates the discrepancy, suggesting that the solar neutrino problem is indeed a real problem. The totality of the data strongly suggests that the simplest astrophysical solutions are ruled out, and that new physics is needed [74]. The most attractive possibility is to assume the existence of neutrino conversions involving very small neutrino masses.

The detection rates in the chlorine and gallium experiments are given as

$$
R_{C l, G a}=\int d E \sigma(E) \mathcal{P}(\mathcal{E}) \sum_{\rangle} \phi_{\rangle}(\mathcal{E}),
$$

where $\mathcal{P}$ is the neutrino survival probability and the sum is over the relevant neutrino sources $\left(i={ }^{7} \mathrm{Be},{ }^{8} \mathrm{~B} \ldots\right)$ and $\sigma(E)$ are the corresponding neutrino cross sections. For the Kamiokande experiment, the detection rate is

$$
R_{k}=\int_{T h} d E\left[\sigma_{\nu_{e}}(E) \mathcal{P}(\mathcal{E})+\sigma_{\nu_{\S}}(\mathcal{E})(\infty-\mathcal{P}(\mathcal{E}))\right] \phi_{\mathcal{B}}(\mathcal{E})
$$

where $\sigma_{\nu_{e}}(E)$ and $\sigma_{\nu_{x}}(E)(x=\mu, \tau)$ are the $\nu_{e}-e$ and $\nu_{x}-e$ elastic scattering cross sections, respectively, and 'Th' stands for the detection energy threshold. In the case of sterile conversion $\sigma_{\nu_{x}}=0$.

The quantity $\mathcal{P}$ depends on the particle physics mechanism used in order to suppress the solar neutrino fluxes.

One of the simplest mechanisms suggested in order to solve the solar neutrino problem is the long-wavelength or just-so oscillation. The $68 \%$ C.L. allowed region of oscillation parameters is displayed in Fig. (7), taken from ref. [75]. 

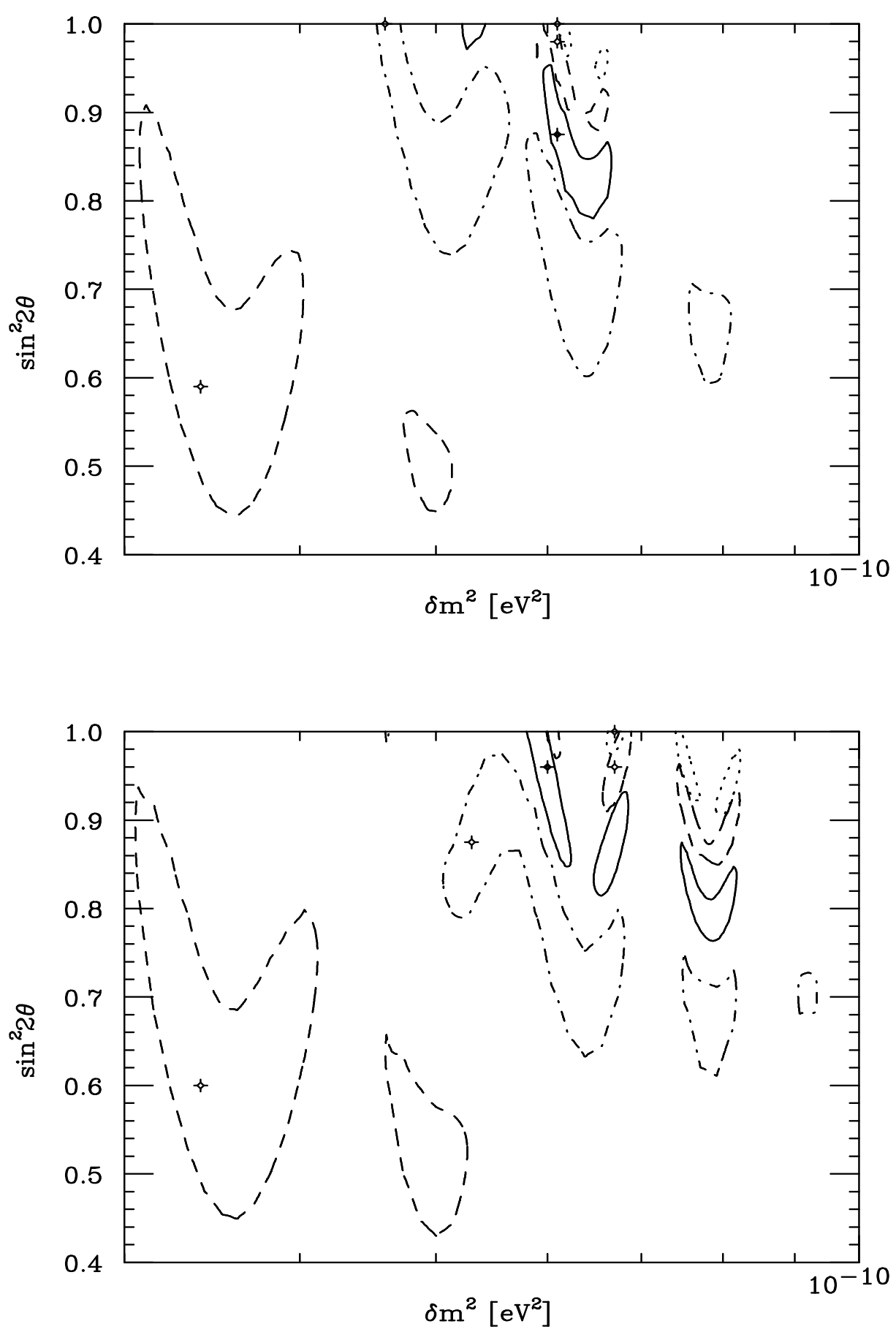

Figure 7: Allowed parameters for active (upper figure) and sterile (lower figure) just-so solar neutrino oscillations. 


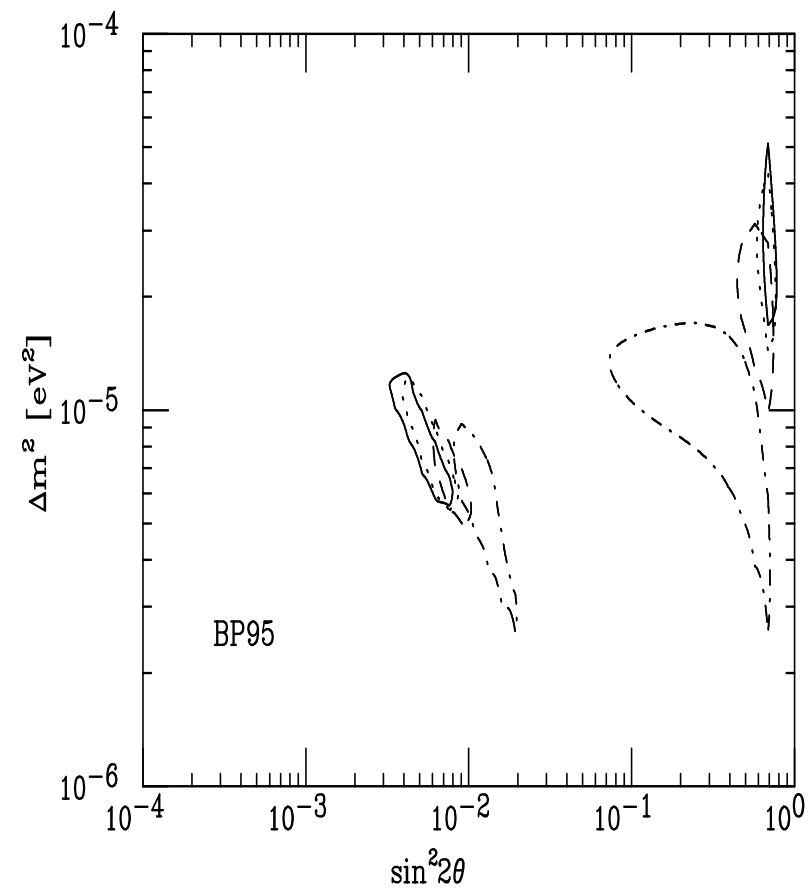

Figure 8: Allowed solar neutrino oscillation parameters for active neutrino conversions.

We now turn to the most elegant and popular solution to the solar neutrino deficit, namely the MSW effect [76]. This takes into account the fact that solar neutrinos, produced mostly close to the solar centre, interact with solar matter before they escape the Sun. In this case Mikheyev and Smirnov discovered in 1985 a beautiful and very simple mechanism of resonantly enhancing the neutrino conversion probabilities. In order to make detailed solar neutrino predictions in the framework of the MSW effect and to determine the required neutrino parameters one needs to integrate the system of neutrino evolution equations describing oscillations in matter of varying density. The required set of solar neutrino parameters $\Delta m^{2}$ and $\sin ^{2} 2 \theta$ are determined through a $\chi^{2}$ fit of the experimental data 0 .

In Fig. (8), taken from ref. [77, one shows the 90\% C.L. areas for the BP95 model for the case of active neutrino conversions. The fit favours the small mixing solution over the large mixing one, due mostly to the larger reduction of the ${ }^{7} \mathrm{Be}$ flux found in the former. Here $\xi$ denotes the assumed level of noise fluctuations in the solar matter density [78], not excluded by the SSM nor by present helioseismology studies. The solid curves are for the standard $\xi=0$ assumption corresponding to a smooth Sun. The regions inside the other curves correspond to the case where matter density fluctuations are assumed. Noise causes a slight shift of $\Delta m^{2}$ towards lower values and a larger shift of $\sin ^{2} 2 \theta$ towards larger values. The corresponding allowed $\Delta m^{2}$ range is $2.5 \times 10^{-6}<\Delta m^{2}<9 \times 10^{-6} \mathrm{eV}^{2}$ instead of $5 \times 10^{-6}<\Delta m^{2}<1.2 \times 10^{-5} \mathrm{eV}^{2}$ in the noiseless case. The large mixing area is less stable, exhibiting a tendency to shift towards smaller $\Delta m^{2}$ and $\sin ^{2} 2 \theta$. For example, if we take $\xi=8 \%$, for the sake of argument, we find

\footnotetext{
${ }^{d}$ For simplicity here we neglect theoretical uncertainties, as well as the details of the neutrino production region and the earth effects.
} 


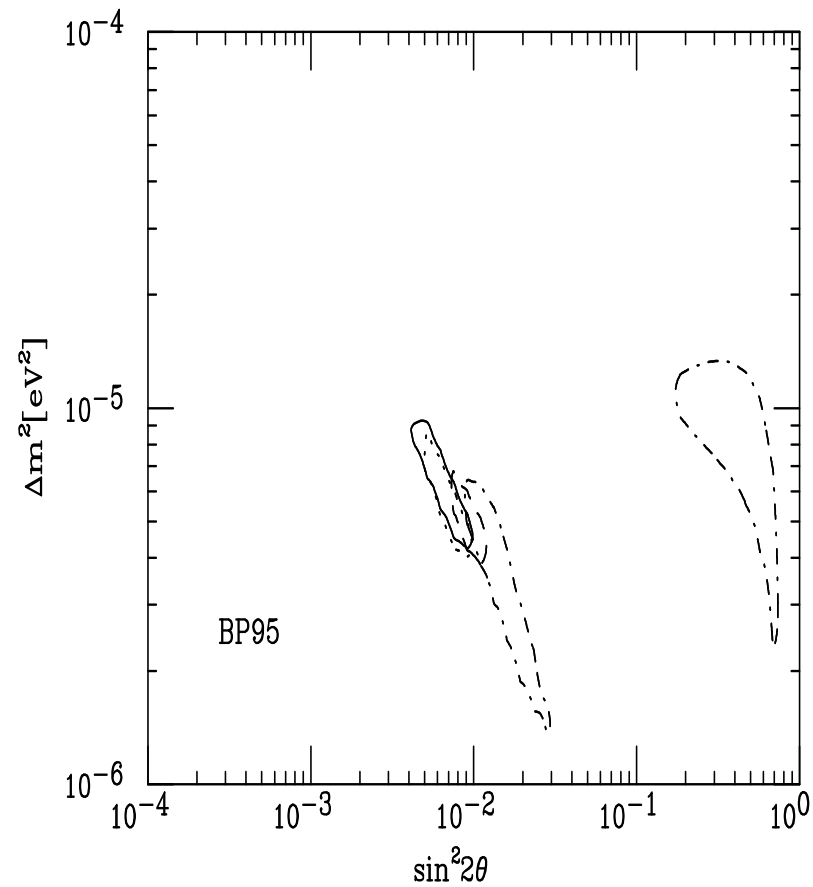

Figure 9: Allowed solar neutrino oscillation parameters for sterile neutrino conversions.

that the small mixing region is much more stable than the large mixing one, even for such a relatively large value of the noise. For details, such as some discussion of other solar models see ref. [77].

The results for the case of sterile solar neutrino conversions are given, also for the BP95 model, in Fig. 9 The fit is now worse than for the active case and excludes, even at 95\% C.L., the large mixing region (in the noiseless case). The presence of matter density noise may restore this region and avoid a conflict with the primordial helium abundance constraints 79 .

As we have seen the ${ }^{7}$ Be neutrinos are the component of the solar neutrino spectrum which is most affected by the presence of matter noise. Therefore the future Borexino experiment, aimed to detect the ${ }^{7}$ Be neutrino flux [80] through the elastic $\nu$ - $e$ scattering should be an ideal tool for studying the solar matter fluctuations. Its potential in "testing" the level of matter density fluctuations in the solar interior through the measurement of the ${ }^{7}$ Be neutrino flux is illustrated in Fig. (4). The solid lines in this figure show the iso-signal contours for the predicted over expected (in the BP95 model) ${ }^{7}$ Be signal and the corresponding $90 \%$ allowed regions lie inside the dashed lines, with the best fit points denoted by a cross. The top figure is for the smooth Sun, while the other corresponds to noise at the $4 \%$ level. One sees that, with sufficiently small statistical errors, the Borexino experiment would have great potential in testing the level of density fluctuations in the solar interior. See ref. [77] for more details. 

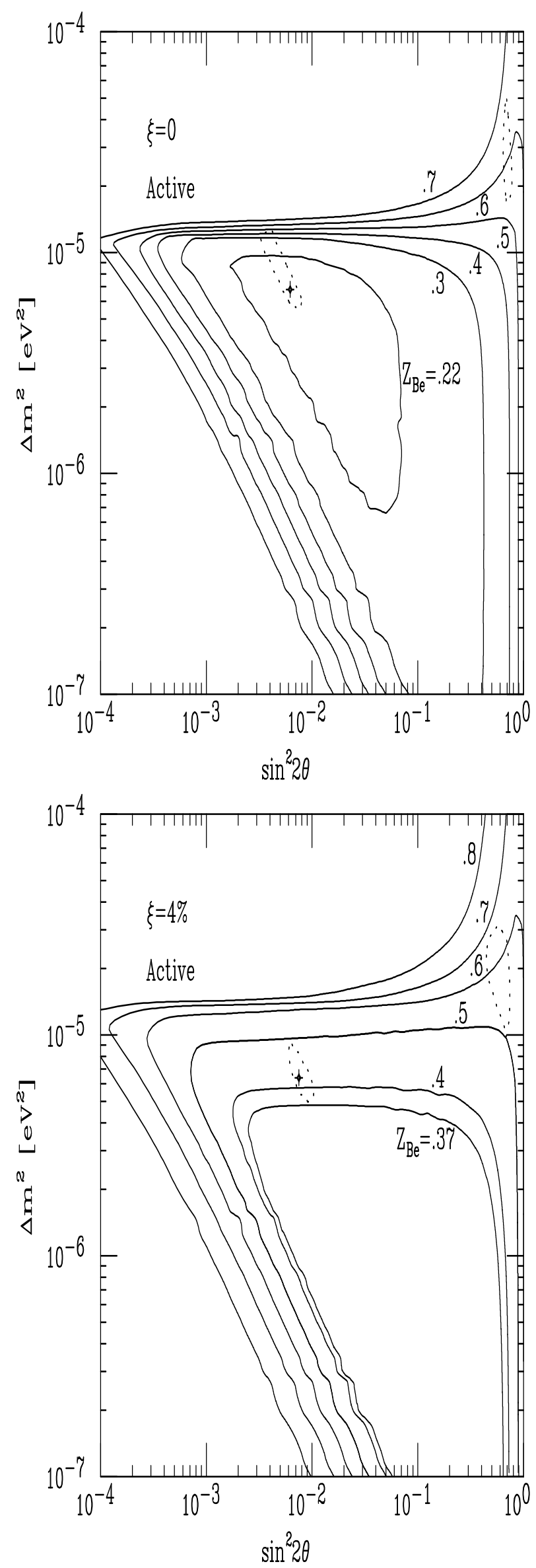

Figure 10: Potential of Borexino to test solar density noise fluctuations. 


\subsubsection{Atmospheric Neutrinos}

Two underground experiments, Kamiokande and IMB, and possibly also Soudan2, have indications which support an apparent deficit in the expected flux of atmospheric $\nu_{\mu}$ 's relative to that of $\nu_{e}$ 's that would be produced from conventional decays of $\pi$ 's, $K$ 's as well as secondary muon decays. Although the predicted absolute fluxes of neutrinos produced by cosmic-ray interactions in the atmosphere are uncertain at the $20 \%$ level, their ratios are expected to be accurate to within 5\%. While some of the experiments, such as Frejus and NUSEX, have not found a firm evidence, it has been argued that there may be a strong hint for an atmospheric neutrino deficit that could be ascribed to neutrino oscillations. It is not our purpose here to give a detailed discussion, specially in view of the controversy between water Cerenkov and iron calorimetry experiments, for that we refer the reader to the experimental reviews in ref. 81. We will stress, however, that Kamiokande results on higher energy neutrinos strengthen the case for an atmospheric neutrino problem due to the observed zenith-angle dependence. The relevant oscillation parameters are shown in ref. [82].

\subsection{Models Reconciling Present Hints.}

Can we reconcile the present hints from astrophysics and cosmology in the framework of a consistent elementary particle physics theory? The above observations suggest an interesting theoretical puzzle whose possible resolutions will now be discussed.

\subsubsection{Three Almost Degenerate Neutrinos}

It is difficult to reconcile these three observations simultaneously in the framework of the simplest seesaw model with just the three known neutrinos. The only possibility to fit these observation in a world with just the three neutrinos of the Standard Model is if all of them have nearly the same mass $\sim 2 \mathrm{eV} 83$.

It is known that the general seesaw models have two independent terms giving rise to the light neutrino masses. The first is an effective triplet vacuum expectation value [6] which is expected to be small in left-right symmetric models [16]. Based on this fact one can in fact construct extended seesaw models where the main contribution to the light neutrino masses $(\sim 2 \mathrm{eV})$ is universal, due to a suitable horizontal symmetry, while the splittings between $\nu_{e}$ and $\nu_{\mu}$ explain the solar neutrino deficit and that between $\nu_{\mu}$ and $\nu_{\tau}$ explain the atmospheric neutrino anomaly [84. 


\subsubsection{Three Active plus One Sterile Neutrino}

The alternative way to fit all the data is to add a fourth neutrino species which, from the LEP data on the invisible $\mathrm{Z}$ width, we know must be of the sterile type, call it $\nu_{s}$. The first scheme of this type gives mass to only one of the three neutrinos at the tree level, keeping the other two massless [7]. In a seesaw scheme with broken lepton number, radiative corrections involving gauge boson exchanges will give small masses to the other two neutrinos $\nu_{e}$ and $\nu_{\mu}$ 85. However, since the singlet neutrino is super-heavy in this case, there is no room to account for the three hints discussed above.

Two basic schemes have been suggested to keep the sterile neutrino light due to a special symmetry. In addition to the sterile neutrino $\nu_{s}$, they invoke additional Higgs bosons beyond that of the Standard Model, in order to generate radiatively the scales required for the solar and atmospheric neutrino conversions. In these models the $\nu_{s}$ either lies at the dark matter scale [86] or, alternatively, at the solar neutrino scale [87]. In the first case the atmospheric neutrino puzzle is explained by $\nu_{\mu} \rightarrow \nu_{s}$ oscillations, while in the second it is explained by $\nu_{\mu} \rightarrow$ $\nu_{\tau}$ oscillations. Correspondingly, the deficit of solar neutrinos is explained in the first case by $\nu_{e} \rightarrow \nu_{\tau}$ conversions, while in the second it is explained by $\nu_{e} \rightarrow \nu_{s}$ oscillations. In both cases it is possible to fit all observations together. However, in the first case there may be a clash with the bounds from big-bang nucleosynthesis. In the latter case the $\nu_{s}$ is very light, at the solar neutrino scale, so that nucleosynthesis limits are satisfied and, if nucleosynthesis limits are taken at face value they single out the small mixing MSW solution uniquely. Moreover, the mixing angle characterising the $\nu_{\mu} \rightarrow \nu_{\tau}$ oscillations is nearly maximal, in nice agreement with ref. [82]. Moreover, it can naturally fit the recent preliminary hints of neutrino oscillations of the LSND experiment 65]. Finally, there is another theoretical possibility is that all active neutrinos are very light, while the sterile neutrino $\nu_{s}$ is the single neutrino responsible for the dark matter [88.

\subsubsection{MeV Tau Neutrino}

A tau neutrino with a mass in the $\mathrm{MeV}$ range is an interesting possibility to consider for two different reasons. On experimental side such a neutrino is within the range of the detectability, for example at a tau-charm factory [43, 89]. On the other hand, if such neutrino decays before the matter dominance epoch, its decay products could then add energy to the radiation thereby delaying the time at which the matter and radiation contributions to the energy density of the universe become equal. Such delay would allow one to reduce the density fluctuations at the smaller scales 90] purely within the standard cold dark matter scenario [91], and could reconcile the large scale fluctuations observed by COBE [66] with the earlier observations such as those 
of IRAS 68 on the fluctuations at smaller scales. An MeV $\nu_{\tau}$ may, however, conflict with the big-bang nucleosynthesis picture [58].

We present a model where an unstable MeV Majorana tau neutrino can naturally reconcile the cold dark matter model (CDM) with cosmological observations of large and small scale density fluctuations and, simultaneously, with data on solar and atmospheric neutrinos. The solar neutrino deficit is explained through long wavelength, so-called just-so oscillations involving conversions of $\nu_{e}$ into both $\nu_{\mu}$ and a sterile species $\nu_{s}$, while atmospheric neutrino data are explained through $\nu_{\mu}$ to $\nu_{e}$ conversions. Future long baseline neutrino oscillation experiments, as well as some reactor experiments should test this hypothesis. The model is based on the spontaneous violation of a global lepton number symmetry at the weak scale. This symmetry plays a key role in generating the cosmologically required decay of the $\nu_{\tau}$ with lifetime $\tau_{\nu_{\tau}} \sim$ $10^{2}-10^{4}$ seconds, as well as the masses and oscillations of the three light neutrinos $\nu_{e}, \nu_{\mu}$ and $\nu_{s}$ required in order to account for solar and atmospheric neutrino data. It also leads to the invisibly decaying Higgs signature that can be searched at LEP and future particle colliders.

\section{Electroweak Symmetry Breaking: The Higgs Sector}

In the Standard Model the spontaneous breaking of the gauge symmetry follows from energetics, namely the minimum of the so-called Higgs potential favours a non-zero value for the scalar field VEV in eq. (囵). This eliminates three of the four degrees of freedom present in the complex scalar doublet $\phi$ in favour of the longitudinal degrees of freedom of the $W^{ \pm}$and the $Z$. The surviving electrically neutral Higgs scalar, the so-called Standard Model Higgs boson, has a mass given by

$$
m_{h} \propto \sqrt{\lambda}\langle\phi\rangle
$$

where $\lambda$ is the quartic coupling in the Higgs potential. A great effort has been devoted in designing a search for the Standard Model Higgs boson. Clearly this is one of the main open questions of the Standard Model [92.

\subsection{Standard Model Higgs}

Unfortunately both the mass and self-coupling strengths of the Higgs boson are undetermined by the theory. There is, however, an upper bound on the Higgs boson mass that follows from requiring that our simple theoretical picture be valid up to a given cutoff scale $\Lambda$, as otherwise a Landau pole would develop in the renormalization group equation describing the evolution of the quartic coupling $\lambda$ in the Higgs potential. This upper bound depends on the top quark mass through the renormalization group equation, as illustrated in Fig. (11). The limit varies 


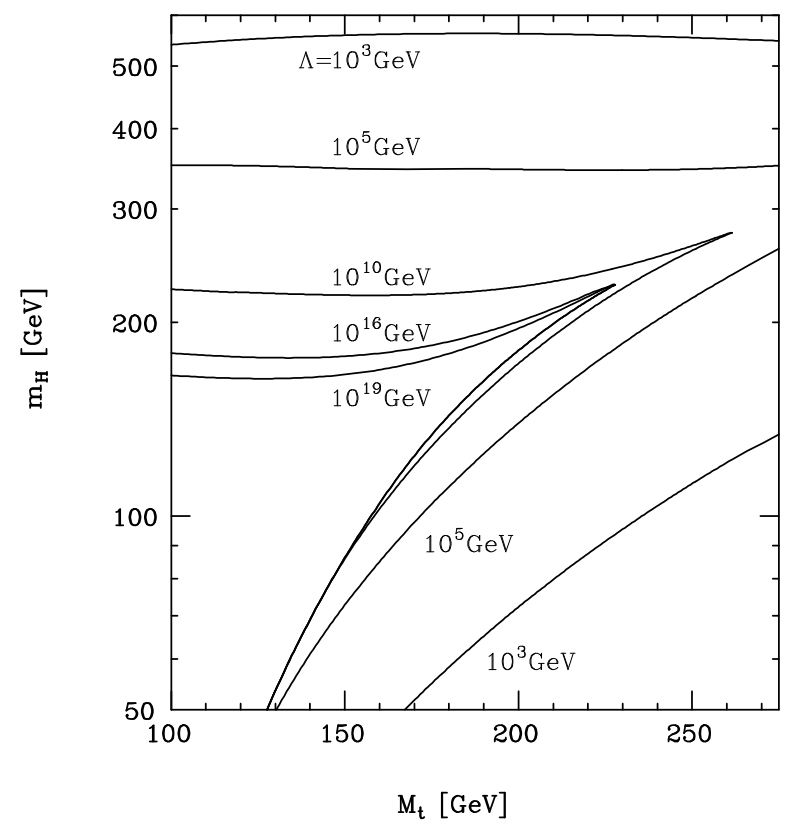

Figure 11: Theoretical Bounds on the Standard Model Higgs Boson.

from $m_{H} \lesssim 600 \mathrm{GeV}$ down to $m_{H} \lesssim 200 \mathrm{GeV}$ if one also assumes that there is no new physics below $M_{\text {Planck } 93 .}$

There is also a theoretical lower bound on the Higgs boson mass obtained by requiring vacuum stability. Since the contribution of the top quark Yukawa coupling to the beta function of the scalar self-coupling, $\lambda$, is negative, the large top quark mass drives $\lambda$ to a negative value, thus destabilizing the Standard Model vacuum. This instability can be avoided by requiring that the Higgs mass be sufficiently large [93]. Indeed a lower bound on the Higgs mass can be obtained by requiring that the Standard Model vacuum be the only stable minimum up to that scale. Requiring of vacuum stability up to the Planck scale gives, for a top quark mass of $175 \mathrm{GeV}$, a lower bound of $130 \mathrm{GeV}$ on the Higgs boson mass [94] If the Higgs mass is lighter than this bound, then the Standard Model must break down at some scale $\Lambda$. At energy scales below $\Lambda$, the physics beyond the Standard Model generally decouples, leaving a low-energy effective theory almost identical to the Standard Model. However, the Higgs boson allows us to probe $\Lambda$ through the above stability constraint on the Higgs mass, and due to the large top quark mass.

By combining these two arguments one obtains for the allowed region for Higgs boson and top quark masses the one delimited by the solid lines in Fig. (11).

This means that if a smaller Higgs boson mass of $100 \mathrm{GeV}$ is discovered then the Standard Model Higgs boson is ruled out if there is no physics below $\Lambda=M_{P l}$. If, on the other extreme, $\Lambda$ is rather close to the $\mathrm{TeV}$ scale, one would expect the lightest Higgs boson to retain all the properties of the so-called Standard Model Higgs boson.

${ }^{e}$ One may refine this limit by requiring only a meta-stable potential with a lifetime longer than the age of the universe. 


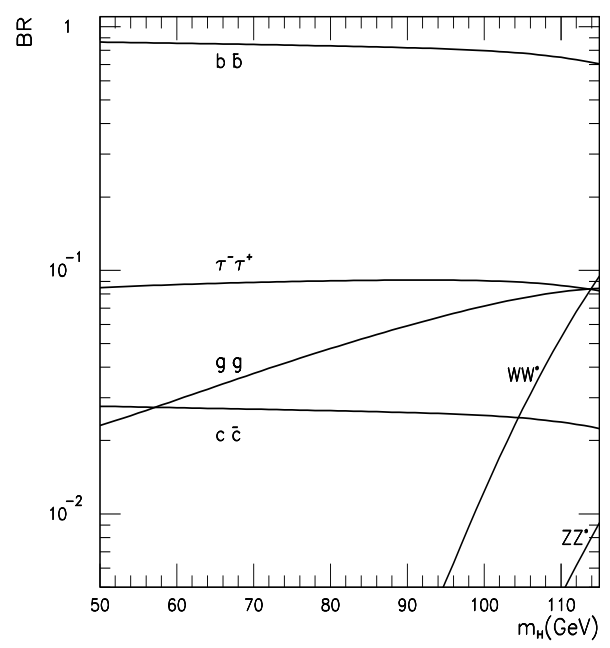

Figure 12: Standard Model Higgs decay branching ratios in mass range accessible at LEP.

Now we turn to the experimental limits on the Higgs boson mass. In order to determine this one needs to know the Higgs boson decays which follow from its interactions with the fermions as well as gauge bosons. The interaction of the physical Higgs with the mass eigenstate fermions is clearly diagonal when written in the same basis that diagonalizes the fermion masses eq. (14). As a result there are no flavour changing neutral currents mediated by Higgs boson exchange in the Standard Model. Moreover, the couplings of the physical Higgs boson to both gauge boson and fermions is proportional to their mass. This leads to a well defined Higgs boson decay pattern, which is of fundamental importance in designing the strategies for Higgs searches at accelerators. Indeed, the Higgs boson will decay mostly to the heaviest kinematically accessible particle, as illustrated in Fig. (12). From the analysis of the data collected at LEP one can place the following lower limit on the Standard Model Higgs boson mass 95

$$
m_{H} \gtrsim 65 \mathrm{GeV}
$$

As illustrated in Fig. (13), if lighter than $\sim 100 \mathrm{GeV}$, the Standard Model Higgs boson should be found at LEP 200 [96]. The minimum required luminosity per experiment, in $\mathrm{pb}^{-1}$, for a $5 \sigma$ Higgs boson discovery is displayed in the solid line of Fig. (13), while the corresponding 95\% C.L. exclusion limit is shown as dashed. Results for other centre-of-mass energies can be found in refs. [96, 97]. Higher Higgs boson masses can be probed at higher centre-of-mass energies, such as expected in the next linear collider (NLC), or or at the LHC. Unfortunately the prospects for finding the Higgs boson in the intermediate mass range between $m_{Z}$ and $2 m_{Z}$ at the LHC are not too optimistic [98]. Above this mass the detection would be very easy, through the 4-lepton signal, as illustrated in Fig. 9, 10, 11 and 12 of ref. 98.

From the above vacuum stability argument it follows that if the Higgs has a mass just above its current experimental limit, then the Standard Model must break down at a scale of roughly a TeV. Since, by definition, the Standard Model assumes that there is no new physics until a 


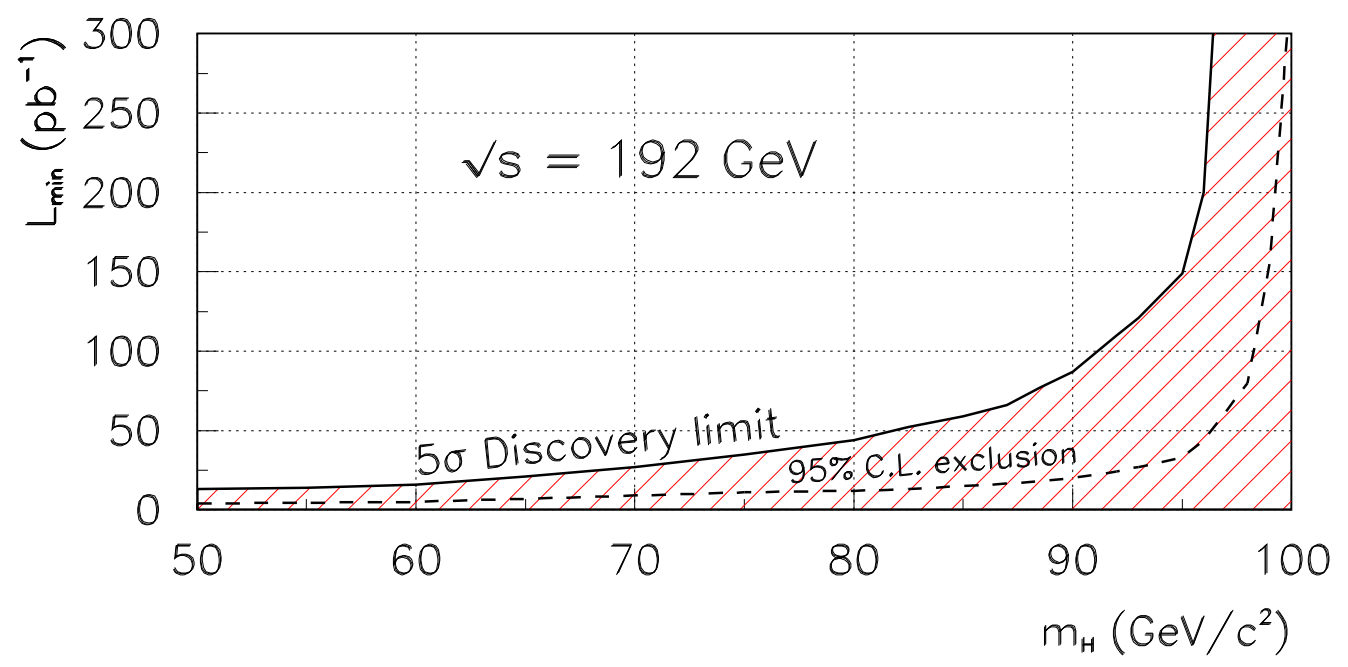

Figure 13: Standard Model Higgs boson search potential at LEP200.

scale of several TeV (at least), it follows that the discovery of a Higgs boson at LEP 200 could, depending on the precise top quark mass, rule out the Standard Model [99.

Let us now move back to experiment and note that one can get indirect experimental information on the Higgs boson mass just on the basis of precision tests of the electroweak theory. In addition to testing the Standard Model, one has the possibility of constraining the value of the Higgs mass, which enters through the radiative corrections to the $Z$ and $W$ boson self-energies. Combining the most recent LEP and SLC electroweak results [100 with the recent top-quark mass measurement at the Tevatron, [4] a weak preference is found for a light Higgs boson mass of order $m_{Z}$ 100. One can illustrate the situation by showing in Fig. (14) a typical chi-square Standard Model fit constraining the Standard Model Higgs mass. The solid line includes all LEP, SLD, $p \bar{p}$ and deep inelastic neutrino data, while the dashed one excludes the measurements of the $\mathrm{Z}$ width into $b \bar{b}$ and $c \bar{c}$. The dotted line corresponds to the LEP data including $R_{b}$ and $R_{c}$. In all cases one includes the direct top mass determination from the Tevatron.

\subsection{Majoron Models}

Many extensions of the lepton sector seek to give masses to neutrinos involve the spontaneous violation of an ungauged U(1) lepton number symmetry. Although the original Majoron proposal was made in the framework of the minimal seesaw model, and required the introduction of a relatively high energy scale associated to the mass of the right-handed neutrinos [25], there are many attractive theoretical alternatives where lepton number is violated spontaneously at the weak scale or lower. Here we are interested precisely in these models, of which there are several examples. In some of these models the neutrinos acquire mass at the tree level [24], while in others it may appear either at one-loop or two-loop level in radiative corrections [26, 101]. 


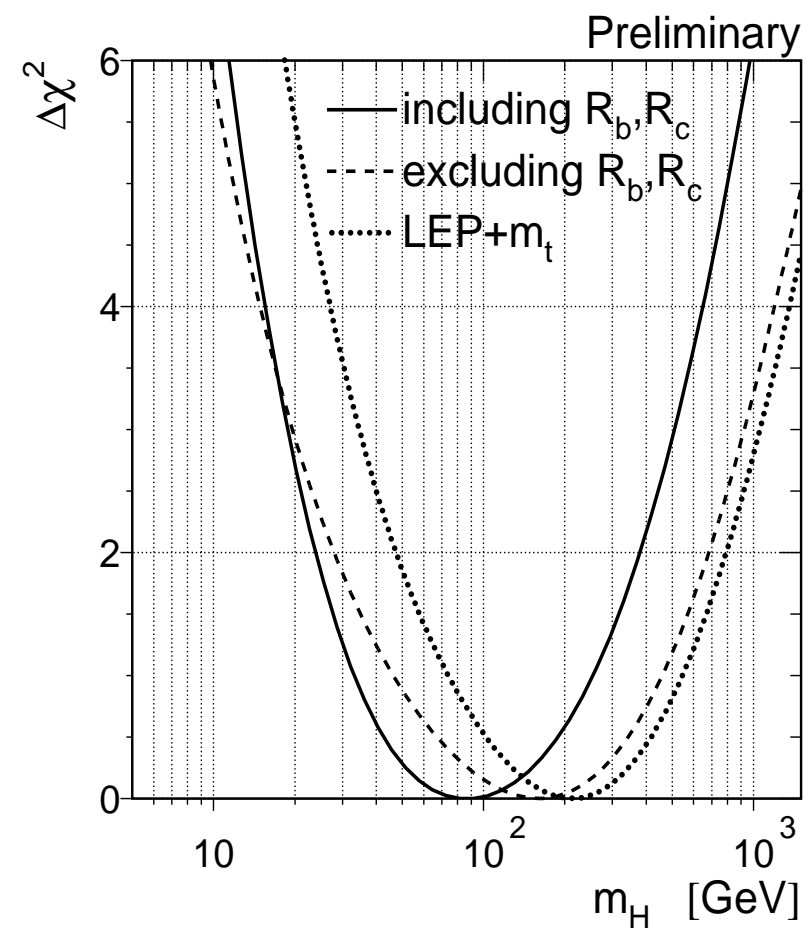

Figure 14: Standard Model Higgs mass determination from precision data.

The main common feature of these models is that the associated neutrino mass vanishes as the scale of spontaneous lepton number violation goes to zero, in sharp contrast to the standard seesaw idea which exhibits an inverse relationship.

All these models imply the existence of a physical Goldstone boson, generically called Majoron. The existence of such Majoron is consistent with the LEP measurements of the invisible $Z$ decay width if the Majoron is (mostly) a singlet under the $S U(2) \otimes U(1)$ gauge symmetry. Although the Majoron has very tiny couplings to matter and gauge bosons, it can have significant couplings to the Higgs bosons, leading to the possibility that the Higgs boson may decay with a substantial branching ratio into the channel [26]

$$
h \rightarrow J+J
$$

Since the Majoron $J$ is weakly coupled to the rest of the particles, once produced in the accelerator, it will escape detection, leading to a missing momentum signal. Since the strategies to search for the Higgs boson depend heavily on its expected decay pattern, the presence of such an invisible decay signal affects them in a very remarkable way.

\subsubsection{Simplest Model}

Models in this class contain one doublet and one singlet Higgs multiplet. They include both models with tree level generation [24], as well as radiative [28]. They are characterized by a very simple form of the scalar potential given by:

$$
V_{N_{1}}=\mu_{\phi}^{2} \phi^{\dagger} \phi+\mu_{\sigma}^{2} \sigma^{\dagger} \sigma+\lambda_{1}\left(\phi^{\dagger} \phi\right)^{2}+\lambda_{2}\left(\sigma^{\dagger} \sigma\right)^{2}+\delta\left(\phi^{\dagger} \phi\right)\left(\sigma^{\dagger} \sigma\right)
$$


Terms like $\sigma^{2}$ are omitted above in view of the imposed $U(1)$ invariance under which we require $\sigma$ to transform non-trivially and $\phi$ to be trivial. Let $\sigma \equiv \frac{w}{\sqrt{2}}+\frac{R_{2}+i I_{2}}{\sqrt{2}}, \phi^{0} \equiv \frac{v}{\sqrt{2}}+\frac{R_{1}+i I_{1}}{\sqrt{2}}$, where we have set $\langle\sigma\rangle=\frac{w}{\sqrt{2}}$ and $\left\langle\phi^{0}\right\rangle=\frac{v}{\sqrt{2}}$. The above potential then leads to a physical massless Goldstone boson, namely the Majoron $J \equiv \operatorname{Im} \sigma$ and two massive neutral scalars $H_{i}(i=1,2)$

$$
H_{i}=\hat{O}_{i j} R_{j}
$$

The mixing $\hat{O}$ can be parametrized as

$$
\hat{O}=\left(\begin{array}{cc}
\cos \theta & \sin \theta \\
-\sin \theta & \cos \theta
\end{array}\right)
$$

mixing angle $\theta$ as well as the Higgs masses $M_{i}^{2}$ are related to the parameters of the potential in the following way:

$$
\begin{aligned}
2 \delta v w & =\left(M_{2}^{2}-M_{1}^{2}\right) \sin 2 \theta \\
2 \lambda_{1} v^{2} & =M_{1}^{2} \cos ^{2} \theta+M_{2}^{2} \sin ^{2} \theta \\
2 \lambda_{2} w^{2} & =M_{2}^{2} \cos ^{2} \theta+M_{1}^{2} \sin ^{2} \theta . \\
\tan 2 \theta & =-\frac{\delta v \omega}{\lambda_{1} v^{2}-\lambda_{2} \omega^{2}}
\end{aligned}
$$

The masses $M_{1,2}^{2}$, the mixing angle $\theta$, and the ratio of two vacuum expectation values $\tan \beta=\frac{v}{w}$ can be taken as independent parameters in terms of which all couplings can be fixed. There are no physical charged Higgs bosons in this case.

The potential in eq. (72) generates the following coupling of $H_{i}$ to the Majoron $J$ :

$$
\mathcal{L}_{J}=\frac{\left(\sqrt{2} G_{F}\right)^{1 / 2}}{2} \tan \beta\left[M_{2}^{2} \cos \theta H_{2}-M_{1}^{2} \sin \theta H_{1}\right] J^{2}
$$

The Higgs decay to two Majorons follows immediately from this equation.

\section{Invisibly Decaying Higgs boson searches in the $e^{+} e^{-} \rightarrow H Z$ channel}

The production and subsequent decay of a Higgs boson which may decay visibly or invisibly via the process $e^{+} e^{-} \rightarrow H Z$ production involves three independent parameters: its mass $M_{H}$, its coupling strength to the $\mathrm{Z}$ boson, normalized by that of the Standard Model, $\epsilon^{2}$, and its invisible decay branching ratio. The LEP searches for various exotic channels can be used in order to determine the regions in parameter space that are already ruled out, as described in ref. [102]. The exclusion contour in the plane $\epsilon^{2}$ vs. $M_{H}$, can be found in ref. 102.

The invisible decay of the Higgs boson may also affect the strategies for searches at higher energies. For example, the ranges of parameters that can be covered by LEP200 searches for various integrated luminosities and centre-of-mass energies have been investigated [103], and the results are illustrated in Fig. (15). Similar analysis can be made for the case of a high 


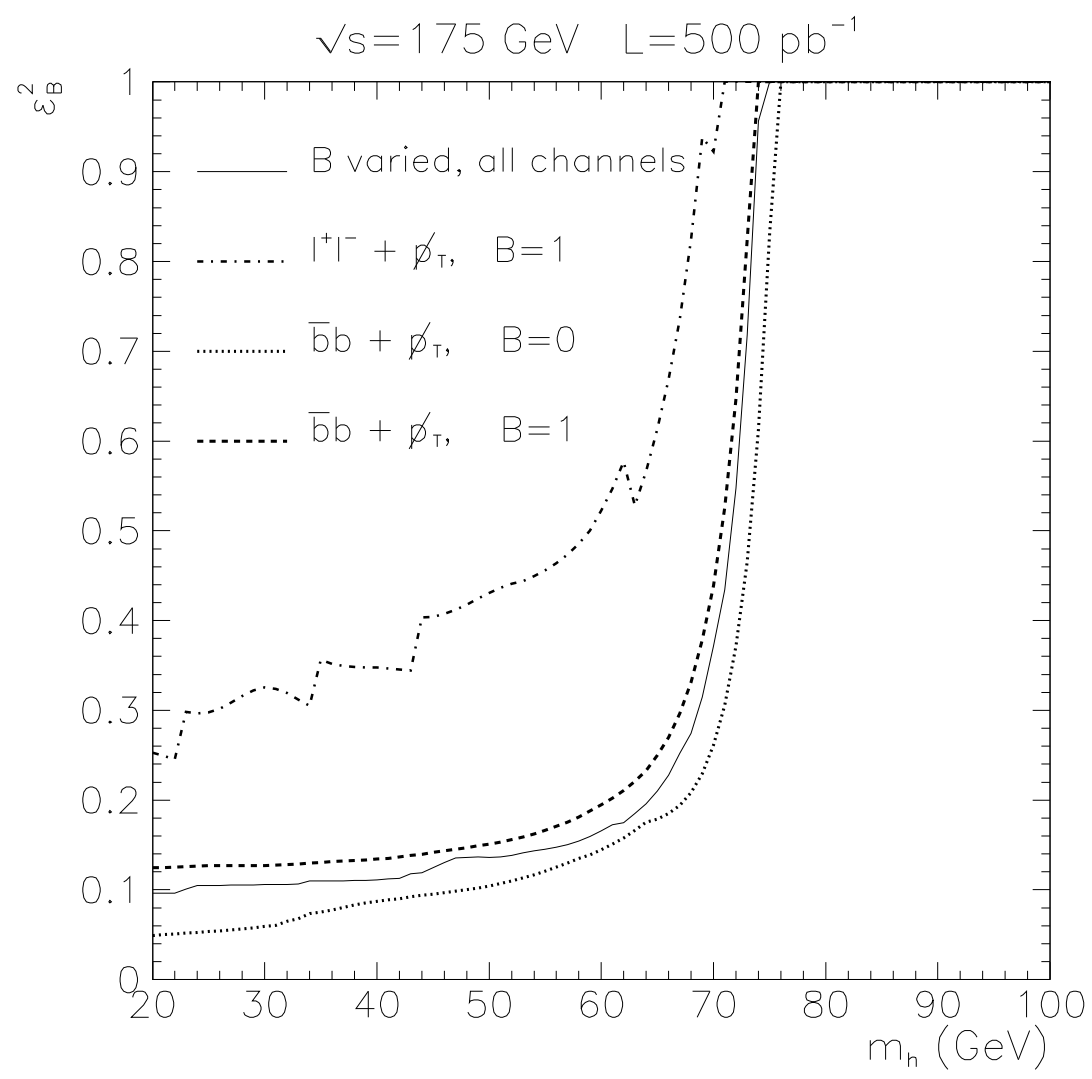

Figure 15: Higgs mass and coupling that can be explored at LEP200 in $e^{+} e^{-} \rightarrow H Z$ production.

energy linear $e^{+} e^{-}$collider (NLC) 104], as well as the LHC [105]. In the latter case the invisible decay has an advantage for searches in the intermediate mass region, namely, that the invisible decay branching ratio can be of order 1 , while the standard $H \rightarrow \gamma \gamma$ decay branching ratio in either the SM or the MSSM is rather small, $\mathcal{O}\left(10^{-3}\right)$. Although it can lead to sizeable signals, the invisible decay has the disadvantage that the Higgs mass can not be reconstructed at a hadron collider. In any case, Higgs boson masses in this range can be probed in less than a year LHC running. However, the NLC would be a cleaner machine for invisibly decaying Higgs boson searches beyond the LEP200 reach.

\subsubsection{Two Scalar Higgs Doublet Extensions}

The models of this class include the Majoron embedding [26] of the original Zee model of radiative neutrino mass generation [27]. In this model a second doublet of scalar bosons is required in order to close the loop diagram yielding the neutrino masses ${ }^{f}$.

The part of the scalar potential containing the neutral Higgs fields is given in this case by

$$
V_{N 2}=\mu_{i}^{2} \phi_{i}^{\dagger} \phi_{i}+\mu_{\sigma}^{2} \sigma^{\dagger} \sigma+\lambda_{i}\left(\phi_{i}^{\dagger} \phi_{i}\right)^{2}+\lambda_{\sigma}\left(\sigma^{\dagger} \sigma\right)^{2}+
$$

\footnotetext{
${ }^{f}$ Another type of model of this type is the model discussed in section 4 . In that model neutrinos get mass at the tree level. The presence of two doublets of scalar bosons, is required by supersymmetry, leading to the existence of a massive CP-odd scalar Higgs boson.
} 


$$
\begin{aligned}
& \lambda_{12}\left(\phi_{1}^{\dagger} \phi_{1}\right)\left(\phi_{2}^{\dagger} \phi_{2}\right)+\lambda_{13}\left(\phi_{1}^{\dagger} \phi_{1}\right)\left(\sigma^{\dagger} \sigma\right)+\lambda_{23}\left(\phi_{2}^{\dagger} \phi_{2}\right)\left(\sigma^{\dagger} \sigma\right) \\
& +\delta\left(\phi_{1}^{\dagger} \phi_{2}\right)\left(\phi_{2}^{\dagger} \phi_{1}\right)+\frac{1}{2} \beta\left[\left(\phi_{1}^{\dagger} \phi_{2}\right)^{2}+\text { h.c. }\right]
\end{aligned}
$$

where a sum over repeated indices $i=1,2$ is assumed. Here $\phi_{1,2}$ are the doublet fields and $\sigma$ corresponds to the singlet carrying nonzero lepton number.

In writing down the above equation, we have imposed a discrete symmetry $\phi_{2} \rightarrow-\phi_{2}$ needed to obtain natural flavour conservation in the presence of more than one Higgs doublets. For simplicity, we assume all couplings and VEVS to be real. Then the conditions for the minimization of the above potential are easy to work out and are given by

$$
\begin{gathered}
\mu_{1}^{2}+v_{1}^{2} \lambda_{1}+\frac{1}{2}\left(\lambda_{12}+\delta\right) v_{2}^{2}+\frac{1}{2} \lambda_{13} v_{3}^{2}+\frac{1}{2} \beta v_{2}^{2}=0 \\
\mu_{2}^{2}+v_{2}^{2} \lambda_{2}+\frac{1}{2}\left(\lambda_{12}+\delta\right) v_{1}^{2}+\frac{1}{2} \lambda_{23} v_{3}^{2}+\frac{1}{2} \beta v_{1}^{2}=0 \\
\mu_{3}^{2}+v_{3}^{2} \lambda_{3}+\frac{1}{2} \lambda_{13} v_{1}^{2}+\frac{1}{2} \lambda_{23} v_{2}^{2}=0
\end{gathered}
$$

These conditions can be used to work out the mass matrix for the Higgs fields. To this end we shift the fields as $(i=1,2)$

$$
\begin{aligned}
\phi_{i} & =\frac{v_{i}}{\sqrt{2}}+\frac{R_{i}+i I_{i}}{\sqrt{2}} \\
\sigma & =\frac{\omega}{\sqrt{2}}+\frac{R_{3}+i I_{3}}{\sqrt{2}}
\end{aligned}
$$

The masses of the CP-even fields $R_{a}(a=1 \ldots 3)$ are obtained from

$$
\mathcal{L}_{\text {mass }}=-\frac{1}{2} R^{T} M_{R}^{2} R
$$

with

$$
M_{R}^{2}=\left(\begin{array}{ccc}
2 \lambda_{1} v_{1}^{2} & \left(\beta+\lambda_{12}+\delta\right) v_{1} v_{2} & \lambda_{13} v_{1} v_{3} \\
\left(\beta+\lambda_{12}+\delta\right) v_{1} v_{2} & 2 \lambda_{2} v_{2}^{2} & \lambda_{23} v_{2} v_{3} \\
\lambda_{13} v_{1} v_{3} & \lambda_{23} v_{2} v_{3} & 2 \lambda_{3} v_{3}^{2}
\end{array}\right)
$$

The physical mass eigenstates $H_{a}$ are related to the corresponding weak eigenstates as

$$
H_{a}=O_{a b} R_{b}
$$

where, $O$ is a $3 \times 3$ matrix diagonalizing $M_{R}^{2}$

$$
O M_{R}^{2} O^{T}=\operatorname{diag}\left(M_{1}^{2}, M_{2}^{2}, M_{3}^{2}\right)
$$

The Majoron is given in this case by $J=I_{3}$. The coupling of the physical Higgses to $J$ follows from eq. (77). As in the previous case, it is possible to express this coupling entirely in terms 
of the masses $M_{a}^{2}$ and the mixing angles characterising the matrix $O$

$$
\begin{aligned}
\mathcal{L}_{J} & =\frac{1}{2} J^{2}\left(2 \lambda_{3} v_{3} R_{3}+\lambda_{13} v_{1} R_{1}+\lambda_{23} v_{2} v_{3} R_{2}\right) \\
& =\frac{J^{2}}{2 v_{3}}\left(M_{R}^{2}\right)_{3 a} R_{a} \\
& =\frac{1}{2}\left(\sqrt{2} G_{F}\right)^{1 / 2} \tan \gamma\left(O^{T}\right)_{3 a} M_{a}^{2} H_{a} J^{2}
\end{aligned}
$$

$\tan \gamma \equiv \frac{V}{v_{3}} ; V=\left(v_{1}^{2}+v_{2}^{2}\right)^{1 / 2}$. We have made use of eq. (85) and eq. (86) in writing the last line.

Unlike in the previous case, now there exists also a massive CP-odd state A, related to the doublet fields as follows

$$
A=\frac{1}{V}\left(v_{2} I_{1}-v_{1} I_{2}\right)
$$

Its mass is given by

$$
M_{A}^{2}=-\beta V^{2}
$$

When $\beta \rightarrow 0$ this pseudoscalar boson becomes massless, as the potential acquires a new symmetry.

\section{Invisibly Decaying Higgs boson searches in the $e^{+} e^{-} \rightarrow H A$ channel}

Due to the existence of two $S U(2) \otimes U(1)$ doublets of scalar bosons, this class of extended Majoron models of neutrino mass predict another mode of production of invisibly decaying Higgs bosons, namely one in which a CP-even Higgs boson is produced in association with a massive CP-odd scalar.

Present LEP1 limits on the corresponding coupling strength parameter were given in ref. [106]. The region of parameters that can be explored at LEP200 is shown in Fig. (16), as a function of the $\mathrm{A}$ and $\mathrm{H}$ masses, for the case of a visibly decaying $\mathrm{A}$ boson and an invisibly decaying $\mathrm{H}$ boson. This figure is taken from ref. [103 which contains extensive discussion of various integrated luminosities and centre-of-mass energy assumptions.

\section{Electroweak Symmetry Breaking: Supersymmetry}

The physics associated to the electroweak breaking sector plays a central role in particle physics. One of the most important physics motivations in favour of supersymmetry is the fact that it is the only symmetry one know which can stabilize the elementary Higgs boson mass with respect to otherwise uncontrollable radiative corrections. These would be expected in any fundamental unified theory including gravity, or simply encompassing the electroweak and strong interactions. Either way one has a very large mass scale - the Planck scale or the grand unification scale - which can mix through loops and destabilize the electroweak scale in eq. (11). 


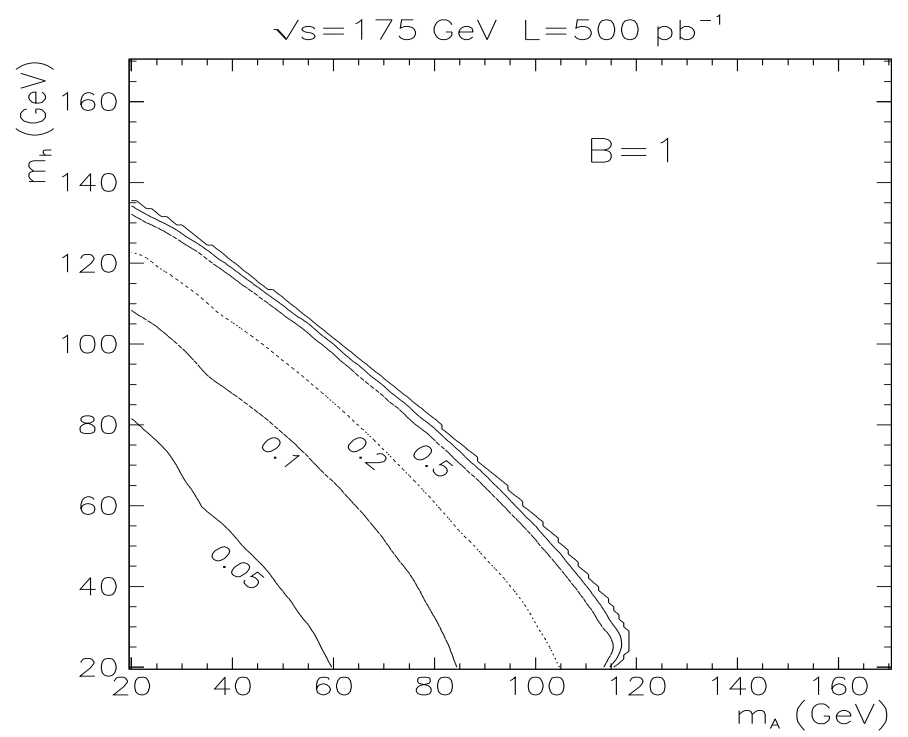

Figure 16: Higgs masses and coupling that can be probed at LEP200 in $e^{+} e^{-} \rightarrow H A$ production.

This so-called hierarchy problem can be solved, at least technically, through supersymmetry [107], to the extent that it holds at $\mathrm{TeV}$ energies and helps to cancel the offending loops.

Supersymmetry is also theoretically attractive as it is the most general symmetry consistent with the basic principles of field theory [108]. Unlike most symmetries discussed in particle physics, that relate particles of the same spin, SUSY relates bosons to fermions, and vice-versa (see table 6).

Finally, the experimental determination of gauge couplings at low energies shows a circumstantial evidence in favour of the existence of SUSY particles f at the TeV scale. This hint is provided by the joining of these gauge couplings at high energies of order of the unification scale $10^{16} \mathrm{GeV}$ [109] as illustrated in Fig. (17), taken from ref. [110]. For all these reasons the study of supersymmetric extensions of the Standard Model has attracted a lot of research effort, including both the theoretical understanding of supersymmetric models and their possible connections with unification schemes, such as provided by string theory as well as the simulation of the expected signals at present and future particle colliders.

\subsection{The MSSM}

The simplest supersymmetric model is the so-called Minimal Supersymmetric Standard Model (MSSM) [111], defined by the particle content given in table 6 . and supplemented by the ad hoc hypothesis that the basic interactions conserve a discrete $\mathrm{R}$ parity $\left(R_{p}\right)$ symmetry, under which all Standard Model particles are even while their partners are odd. The presence of two doublets of Higgs superfields is required by supersymmetry, anomaly cancellation, and in order

${ }^{g}$ For definiteness, one assumes here those present in the so-called MSSM. 


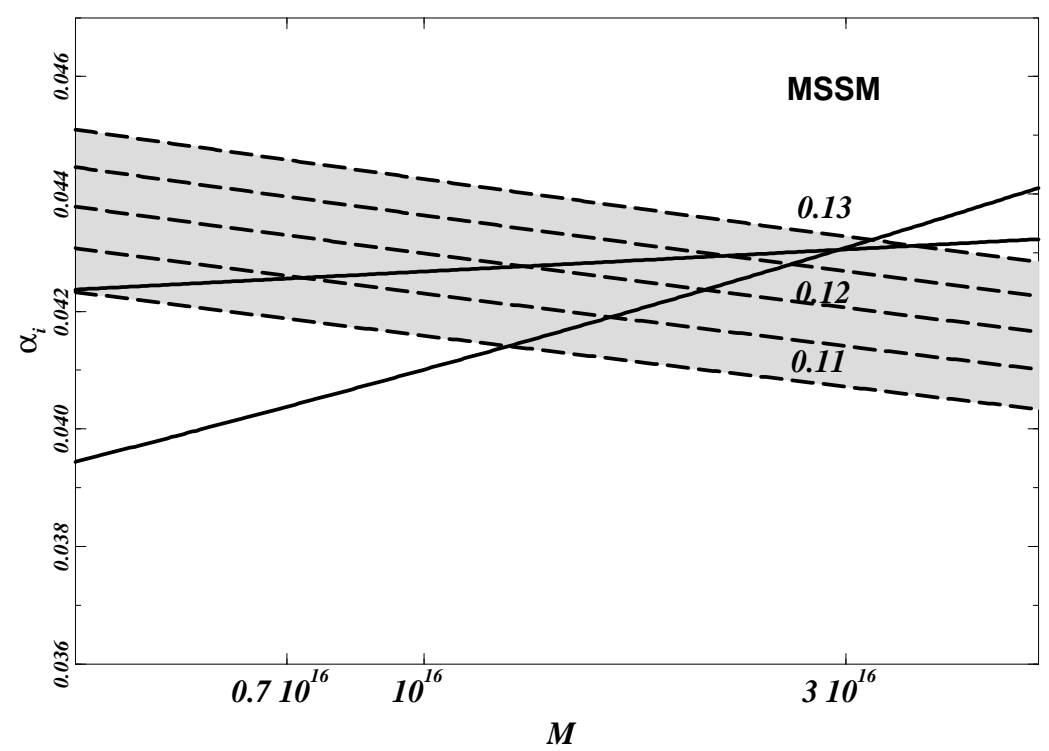

Figure 17: Gauge coupling unification in the MSSM.

\begin{tabular}{||lc|cl||}
\hline Vector & Supermultiplet & Chiral & Supermultiplet \\
\hline$J=1$ & $J=1 / 2$ & $J=1 / 2$ & $J=0$ \\
\hline$g$ & $\tilde{g}$ & $Q_{L}, U_{L}^{c}, D_{L}^{c}$ & $\tilde{Q}_{L}, \tilde{U}_{L}^{c}, \tilde{D}_{L}^{c}$ \\
$W^{ \pm}, W^{0}$ & $\tilde{W}^{ \pm}, \tilde{W}^{0}$ & $L_{L}, E_{L}^{c}$ & $\tilde{L}_{L}, \tilde{E}_{L}^{c}$ \\
$B$ & $\tilde{B}$ & $\tilde{H}_{d}, \tilde{H}_{u}$ & $H_{d}, H_{u}$ \\
\hline
\end{tabular}

Table 6: MSSM multiplet content. 
to give masses to both up and down-type charged fermions.

With this assumption the MSSM is characterised by the following superpotential,

$$
W_{0}=\varepsilon_{a b}\left[h_{i j} \hat{L}_{i}^{a} \hat{H}_{1}^{b} \hat{E}_{j}^{c}+h_{i j}^{\prime} \hat{Q}_{i}^{a} \hat{H}_{1}^{b} \hat{D}_{j}^{c}+h_{i j}^{\prime \prime} \hat{Q}_{i}^{a} \hat{H}_{2}^{b} \hat{U}_{j}^{c}+\mu \hat{H}_{1}^{a} \hat{H}_{2}^{b}\right]
$$

For our subsequent discussion we need the chargino and neutralino mass matrices. The form of the chargino mass matrix is given by

\begin{tabular}{c|cc} 
& $\tilde{H}_{u}^{+}$ & $-i \tilde{W}^{+}$ \\
\hline$\tilde{H}_{d}^{-}$ & $\mu$ & $\sqrt{2} g_{2} v_{d}$ \\
$-i \tilde{W}^{-}$ & $\sqrt{2} g_{2} v_{u}$ & $M_{2}$
\end{tabular}

Two matrices $\mathrm{U}$ and $\mathrm{V}$ are needed to diagonalize the $2 \times 2$ (non-symmetric) chargino mass matrix

$$
\begin{aligned}
& \chi_{i}^{+}=V_{i j} \psi_{j}^{+} \\
& \chi_{i}^{-}=U_{i j} \psi_{j}^{-}
\end{aligned}
$$

where $\psi_{j}^{+}=\left(\tilde{H}_{u}^{+},-i \tilde{W}^{+}\right)$and $\psi_{j}^{-}=\left(\tilde{H}_{d}^{-},-i \tilde{W}^{-}\right)$.

On the other hand the neutralino mass matrix is $4 \times 4$ and has the following form

\begin{tabular}{c|cccc} 
& $\tilde{H}_{u}$ & $\tilde{H}_{d}$ & $-i \tilde{W}_{3}$ & $-i \tilde{B}$ \\
\hline$\tilde{H}_{u}$ & 0 & $-\mu$ & $-g_{2} v_{u}$ & $g_{1} v_{u}$ \\
$\tilde{H}_{d}$ & $-\mu$ & 0 & $g_{2} v_{d}$ & $-g_{1} v_{d}$ \\
$-i \tilde{W}_{3}$ & $-g_{2} v_{u}$ & $g_{2} v_{d}$ & $M_{2}$ & 0 \\
$-i \tilde{B}$ & $g_{1} v_{u}$ & $-g_{1} v_{d}$ & 0 & $M_{1}$
\end{tabular}

This matrix is diagonalized by a $4 \times 4$ unitary matrix $\mathrm{N}$,

$$
\chi_{i}^{0}=N_{i j} \psi_{j}^{0}
$$

where $\psi_{j}^{0}=\left(\tilde{H}_{u}, \tilde{H}_{d},-i \tilde{W}_{3},-i \tilde{B}\right)$, (the indices $i$ and $j$ run from 1 to 4$)$.

In the above two equations $M_{1,2}$ denote the supersymmetry breaking gaugino mass parameters and $g_{1,2}$ are the $S U(2) \otimes U(1)$ gauge couplings divided by $\sqrt{2}$. We assume the canonical relation $M_{1} / M_{2}=\frac{5}{3} \tan ^{2} \theta_{W}$. Typical values for the SUSY parameters $\mu, M_{2}$ and $\tan \beta$ lie in the range given by

$$
-1000 \leq \frac{\mu}{\mathrm{GeV}} \leq 1000 ; 20 \leq \frac{M}{\mathrm{GeV}} \leq 1000 ; 1 \lesssim \tan \beta \lesssim 40
$$


The bilinear term may be replaced by a cubic term of the form $h_{0} H_{u} H_{d} \Phi$. In this case the effective Higgsino mixing parameter $\mu$ is given as $\mu=h_{0}\langle\Phi\rangle$, where $\langle\Phi\rangle$ is the VEV of the appropriate singlet scalar.

Adding the soft supersymmetry breaking scalar mass terms to the supersymmetric gauge interactions dictated by table 6 (D terms) and the supersymmetric Yukawa interactions following from eq. (92) one can write the scalar potential characterising the MSSM. Its general form may be written schematically as

$$
V_{M S S M}=\sum_{i}\left|\frac{\partial W}{\partial z_{i}}\right|^{2}+\tilde{m}_{0}\left[A W_{3}+B W_{2}+h . c .\right]+\sum_{i} \tilde{m}_{i}^{2}\left|z_{i}\right|^{2}+\alpha\left(\left|H_{u}\right|^{2}-\left|H_{d}\right|^{2}-|\tilde{\nu}|^{2}\right)^{2}
$$

where $W_{3}$ and $W_{2}$ denote the cubic and quadratic parts of the superpotential, $\alpha \equiv \frac{g^{2}+g^{\prime 2}}{8}$ and $z_{i}$ denotes any neutral scalar field in the theory. The parameter $\mathrm{A}$ is the cubic soft breaking parameter and $\mathrm{B}=\mathrm{A}-1$ is the corresponding quadratic one 111].

One can show that supersymmetry brings in an attractive possibility to spontaneously break the electroweak symmetry radiatively [112], through renormalization effects from the unification scale down to low energies. Alternatively, assuming colour and electric charge conservation, one can show that the presence of a linear superpotential term in addition to the cubic $h_{0} H_{u} H_{d} \Phi$ term allows for the possibility of breaking the electroweak symmetry at the tree level [113].

\subsection{The MSSM Higgs Sector}

A complete discussion of the MSSM Higgs sector is totally outside the scope of these lectures. For extensive discussions see ref. [114]. Here we will confine ourselves to a very brief summary of the situation, with emphasis on the prospects for Higgs searches at future colliders. For this we will borrow many results from ref. [97.

Due to the necessary presence of two Higgs boson doublets in the MSSM implies that there are two physical CP-even neutral Higgs scalars (h, H), a CP-odd neutral scalar particle, A, and a physical electrically charged scalar boson $H^{ \pm}$. At the tree level the mass of the lightest CP-even neutral Higgs boson $h$ can be calculated in terms of two parameters, which may be chosen as $m_{A}$ and the ratio of Higgs VEVS $\tan \beta$ [114.

Due to the special structure of the MSSM Higgs potential, there is an upper bound on the lightest CP even Higgs boson mass. At the tree level, this bound is exactly the $\mathrm{Z}$ mass. However, it is sensitive to radiative corrections, which are depend on the soft supersymmetry breaking parameters 115 .

The full one-loop radiatively corrected $h$ mass is given in refs. [116 and [117. A simple procedure for accurately approximating $m_{h}$ was described by Haber 118. The dominant 


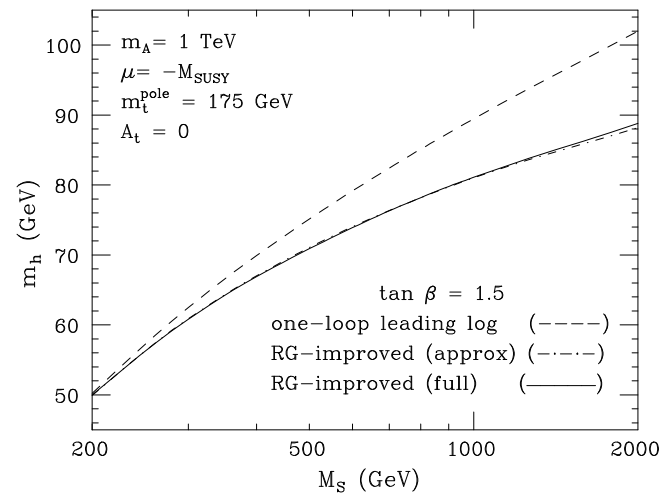

Figure 18: Radiatively corrected MSSM lightest Higgs mass versus SUSY scale.

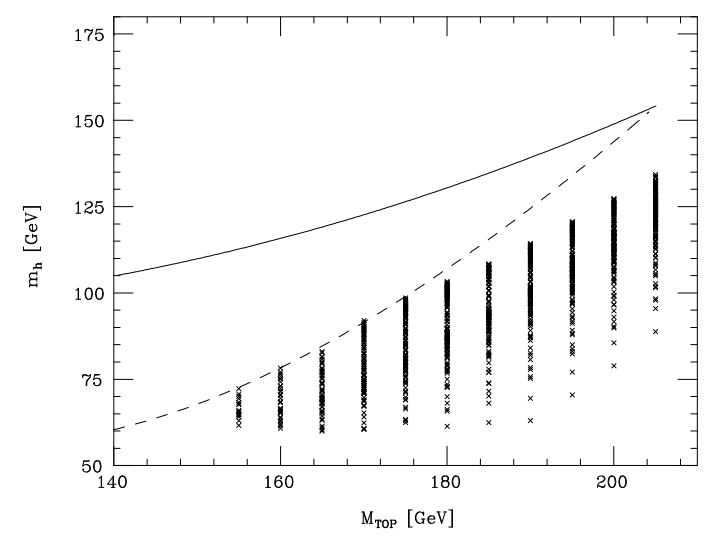

Figure 19: Radiatively corrected MSSM lightest Higgs mass versus $m_{t}$.

radiative corrections to $m_{h}$ arise from an incomplete cancellation of virtual top-quark and topsquark loops. The two top-squark masses $\left(M_{\widetilde{t_{1}}}\right.$ and $\left.M_{\widetilde{t}_{2}}\right)$ are obtained by diagonalizing the corresponding $2 \times 2$ top-squark squared-mass matrix.

We assume that the ratio of Higgs VEVS lies in the range $1 \lesssim \tan \beta \lesssim \frac{m_{t}}{m_{b}}$ and that the scale characterizing supersymmetry breaking $M_{S}$ is less than $2 \mathrm{TeV}$. This scale can be roughly regarded as a common supersymmetric scalar mass. A large $M_{S}$ value takes into account the possibility of large radiative corrections to the lightest CP even Higgs boson mass. We used a top quark mass in the range $m_{t}=175 \pm 35 \mathrm{GeV}$ which generously covers the region indicated by the recent experimental data from the Tevatron. In Fig. (18) we illustrate the dependence of the radiatively corrected lightest CP-even Higgs mass with respect to $M_{S}$ for $\tan \beta=1.5$. The one-loop leading logarithmic computation is compared with the RG-improved result which was obtained by numerical analysis and by using the simple analytic result of ref. [118.

The dependence of the upper bound on the lightest CP-even Higgs boson mass in the MSSM with respect to the top quark mass is given by the solid line in Fig. (19). The dashed line shows the corresponding result for the special case of $b-\tau$ unification under several assumptions, explained in ref. [117]. The complete spectrum of MSSM scalar boson masses, including the h, $\mathrm{H}, \mathrm{A}$ and $H^{ \pm}$masses is shown in Fig. (20) from ref. [117. The dashed, solid and dot-dashed 


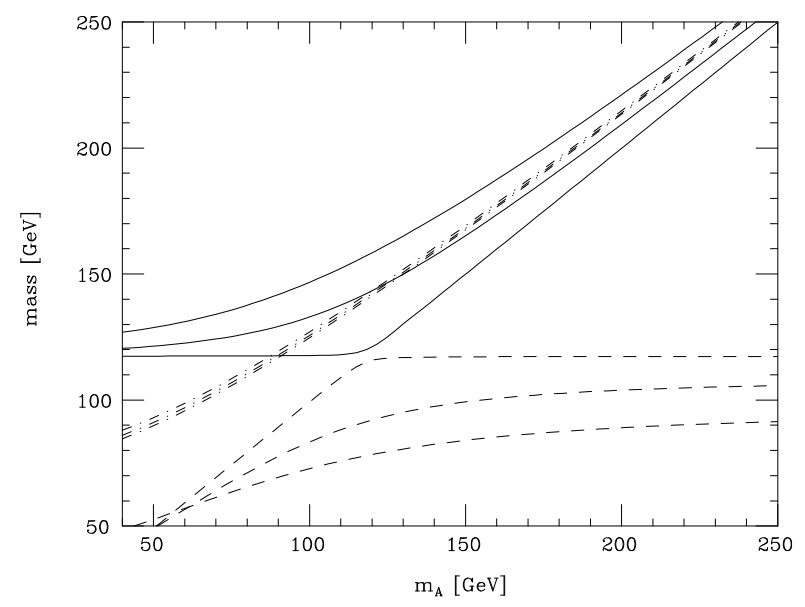

Figure 20: Radiatively corrected MSSM Higgs boson masses.

lines refer to h, $\mathrm{H}$ and $H^{ \pm}$masses respectively. Here one has assumed $m_{t}=175 \mathrm{GeV}, M_{S}=1$ $\mathrm{TeV}, A=-\mu=M_{S}$ and three typical $\tan \beta$ values, 1.6, 3 and 30 (for $\mathrm{h}$ from bottom to top and for $\mathrm{H}$ the other way around). The region of interest is above $40 \mathrm{GeV}$, which is roughly the lower limit on the A mass accessible at LEP1. On the other hand, one sees that for $m_{A}$ above $200 \mathrm{GeV}$ or so there is a very slow variation in $m_{h}$.

The MSSM Higgs boson discovery contours at LEP200 are illustrated in Fig. (21), from ref. [97. This plot corresponds to centre-of-mass energies $192 \mathrm{GeV}$, substantially better for Higgs bosons searches at LEP than $175 \mathrm{GeV}$, and for three stop quark mixing assumptions $A_{t}=0$ and $|\mu| \ll M_{S}$ (no mixing), $A_{t}=M_{S}$ and $\mu=-M_{S}$ (typical mixing), and $A_{t}=\sqrt{6} M_{S}$ and $|\mu| \ll M_{S}$ (maximal mixing), with $M_{S}=1 \mathrm{TeV}$.

\subsection{Limits on SUSY Particles}

As a result of the assumption that $\mathrm{R}$ parity is conserved, the interactions of the MSSM are such that all SUSY particles must be only produced in pairs, with the lightest of them (LSP) being absolutely stable. We take this as a defining feature of the MSSM

So far all searches for supersymmetric particles have been negative. The best existing search site for the weakly interacting SUSY particles is the LEP accelerator. The most recent results follow from searches performed at 130 and $136 \mathrm{GeV}$ centre-of-mass energies and supersede some of the previous LEP1 results.

The basic theoretical considerations involved in the analysis are:

\footnotetext{
${ }^{h}$ The LSP has been suggested as a natural candidate for the cold dark matter (CDM) of the universe. Thus, in addition to searches at accelerators, several methods of detection at underground installations have been suggested. However, as we already saw, the combined COBE and IRAS data do not favour a pure CDM model of structure formation.
} 


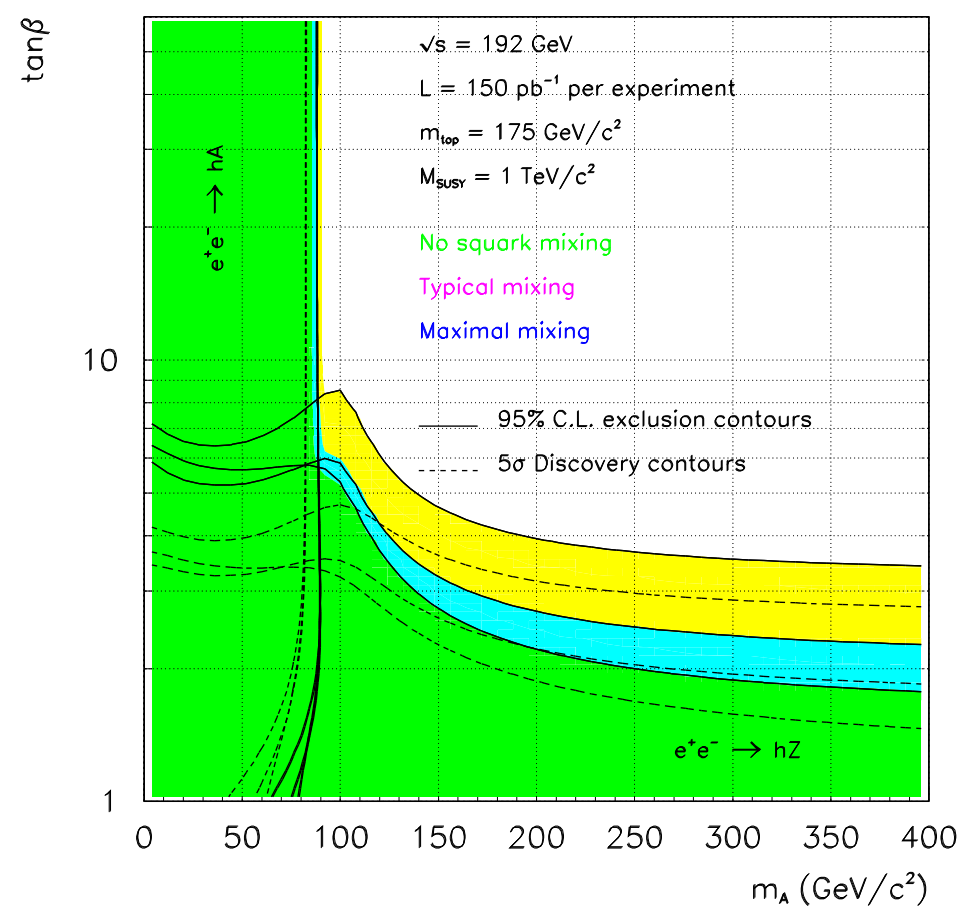

Figure 21: MSSM Higgs boson discovery contours at LEP200.

- The chargino production cross section in $e^{+} e^{-}$collisions has both s-channel Z-mediated as well as t-channel sneutrino- mediated contributions, and may be in the few picobarn range in the $130 \mathrm{GeV}$ energy region.

- Neutralino production also receives a selectron-mediated t-channel contribution. Due to the $\mathrm{R}$ parity conservation hypothesis the $\chi \chi$ production channel does not lead to a visible signal, and one looks for events caused by $\chi \chi^{\prime}$, where $\chi^{\prime}$ decays to $\chi f \bar{f}$, f being any kinematically accessible fermion. This process will lead to acoplanar jets and lepton events with a substantial amount of missing momentum.

- Above the Z peak the highest charged slepton production cross section is for the selectron case, when the LSP is gaugino-like. For the smuons and staus there is no t-channel contribution to the cross section. The decay $\tilde{e}^{ \pm} \rightarrow e^{ \pm}+\chi$ will give rise to dilepton + missing momentum events.

From the non-observation of acoplanar lepton pairs, hadronic events with isolated leptons, hadronic events with missing energy, and acoplanar jet topologies, the Aleph collaboration has recently placed the following limits [95]:

- The new LEP run can be used in order to improve the limit on the lightest chargino mass. Here we quote the result of the Aleph collaboration:

$$
m_{\chi^{ \pm}} \gtrsim 65 \mathrm{GeV}
$$




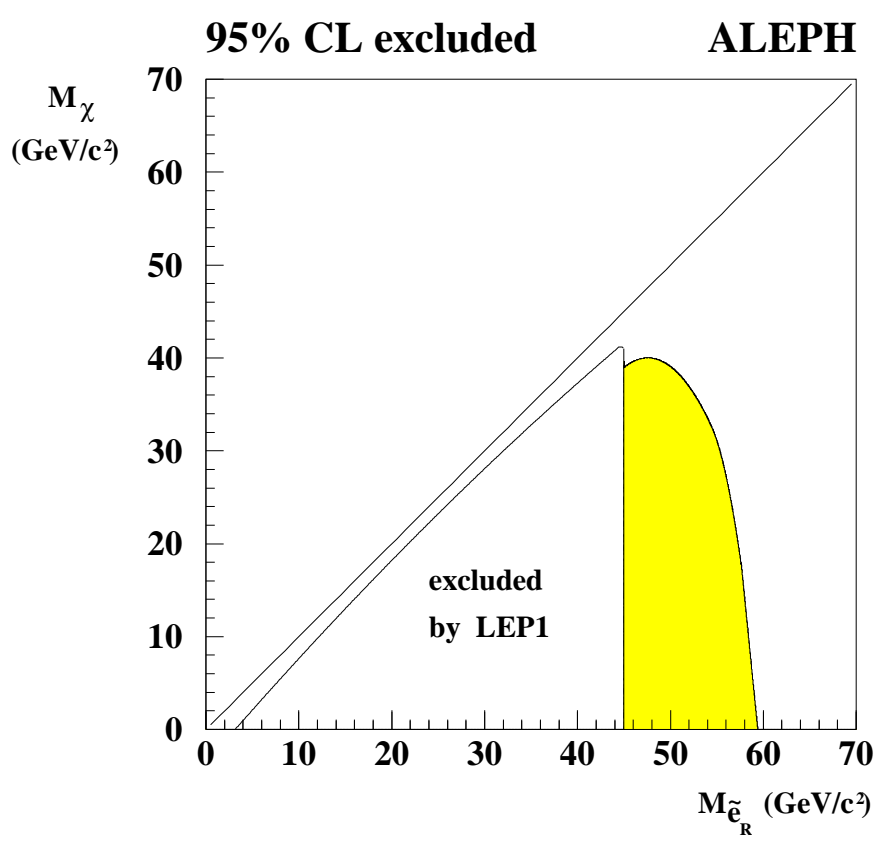

Figure 22: Limits on neutralino and selectron masses in the MSSM.

If the chargino is mostly gaugino this assumes that the sneutrino mass exceeds $200 \mathrm{GeV}$ and, when it is mostly Higgsino, it assumes that the chargino-neutralino mass difference exceeds $10 \mathrm{GeV}$.

- The searches for neutralinos at Aleph lead to the hatched excluded region displayed in Fig. (22), for the case $\mu=1 \mathrm{TeV}$ and $\tan \beta=2$. Note, however, that there is no model independent limit on the lightest neutralino. It heavily depends on the assumption of universal soft-breaking gaugino masses and on the value of the selectron mass. The limits also substantially depend on the assumed decay modes of the heavier neutralino.

- Searches for dilepton + missing momentum events have been performed by the LEP collaborations. The recent Aleph data give the following limit 95]

$$
m_{\ell^{ \pm}} \gtrsim 60 \mathrm{GeV}
$$

which has improved over the previous LEP1 results, but is still worse than previous Tristan limits from single $\gamma$ searches. For the smuons and staus there is no improvement over the $45 \mathrm{GeV}$ LEP1 limit. For sneutrinos, the limit is worse than for charged sleptons.

The limits on squark and gluino masses come mostly from hadron collisions [119. These limits are correlated. For a very heavy gluino, one has $m_{\tilde{q}} \gtrsim 100 \mathrm{GeV}$ for the lower limit on the squarks, with a weaker limit on the top squark. On the other hand, in the limit of very heavy squarks one gets $m_{\tilde{g}} \gtrsim 140 \mathrm{GeV}$ as the corresponding limit on the gluino mass. The allowed region in squark-gluino masses is illustrated in Fig. (23), taken from ref. [119]. The limits given depend 


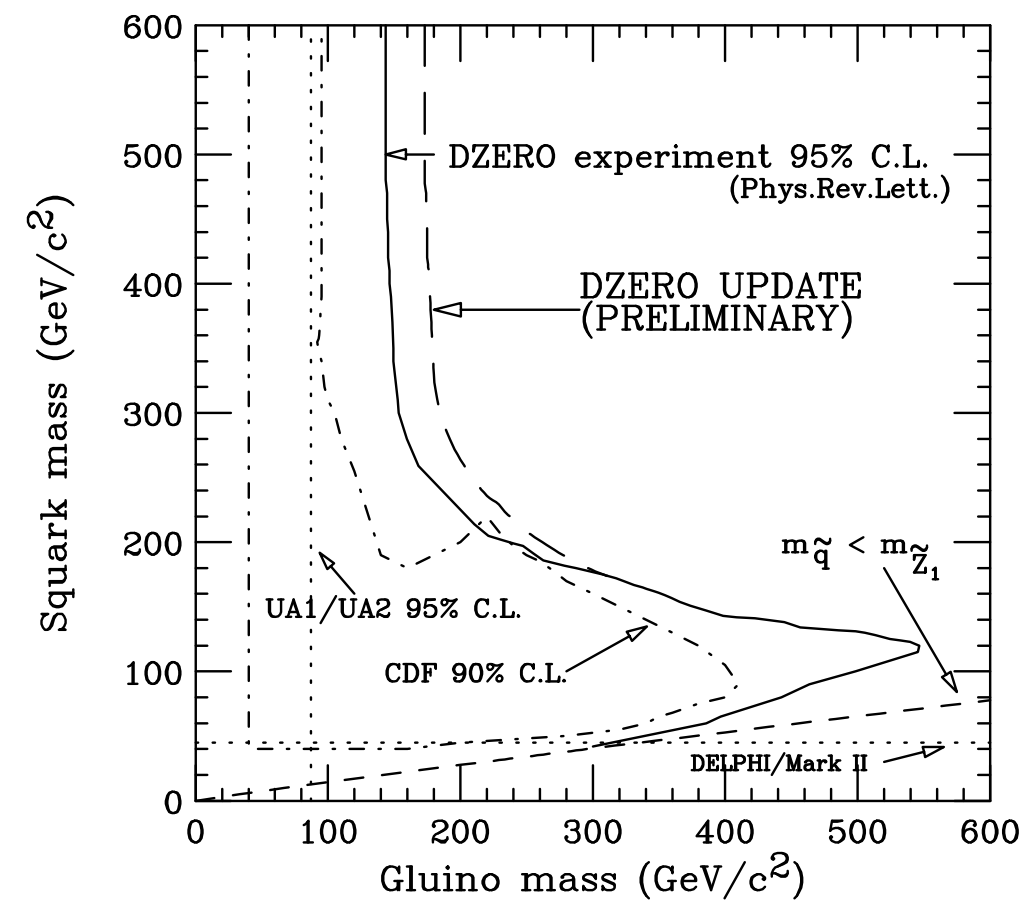

Figure 23: Tevatron limits on squark and gluino masses in the MSSM.

on simplifying assumptions, and some of them may become stronger if one adopts specific parameter choices in the MSSM. On the other hand, they may get weaker in extended models.

The limits for SUSY fermion searches may be combined in order to determine the shape of the corresponding allowed region of region of SUSY parameters $\mu$ and $M_{2}$, for given choices of the ratio of Higgs doublet VEVS $\tan \beta$, as shown in Fig. (24). The region excluded by the chargino search is the shaded region, while the dashed line indicates the previous LEP1 region. The slepton masses are assumed to be $500 \mathrm{GeV}$. The dark area corresponds to the (unlikely) case that the chargino is lighter than all neutralinos. The searches for neutralinos at Aleph lead to the hatched excluded region displayed in Fig. (22), for the case $\mu=1 \mathrm{TeV}$ and $\tan \beta=2$. Additional region of parameter space will become accessible to further searches at higher LEP energies and are eagerly awaited for.

In short, one concludes that there is still a very large domain of parameters where SUSY would be a meaningful symmetry, in terms of being a solution of the hierarchy problem [120], and where its effects could still have been missed. From this point of view it is of great interest to look for its possible effects at higher energies, such as will be accessible at the large hadron collider (LHC) and other future elementary particle accelerators such as the next linear collider (NLC).

An important assumption underlying all SUSY searches conducted so far, is that of R parity conservation. This assumption dictates that all SUSY particles must be produced in pairs, the lightest of these (LSP), typically a neutralino, being absolutely stable. Thus the signal associated to the LSP is missing momentum. These properties have been taken as the basis of 


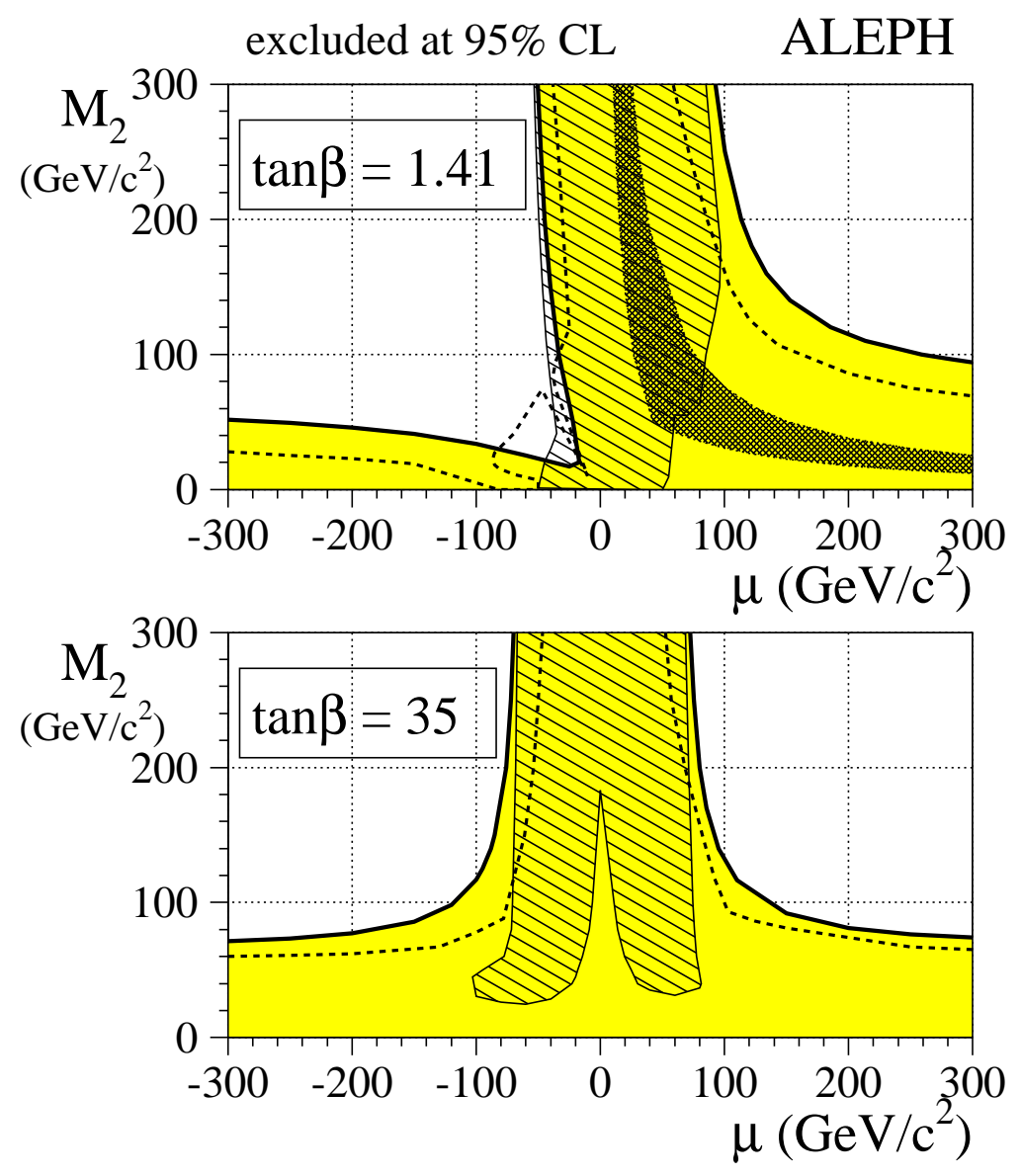

Figure 24: Presently allowed region of MSSM parameters.

all searches of SUSY particles.

Unfortunately there is no clue as to how SUSY is realized. Nobody knows the origin of the R parity symmetry and whether it is indeed a necessary requirement to impose on supersymmetric extensions of the Standard Model. Therefore there is no firm theoretical basis for the MSSM - it is no more than an ansatz, which is the most popular mainly because of its simplicity. Whether or not $\mathrm{R}$ parity is conserved is an important dynamical issue. However, for all we know so far, $\mathrm{R}$ parity conservation may very well break down at some level.

Present SUSY particle search strategies are inadequate for the analysis of extended models where SUSY is realized with broken $\mathrm{R}$ parity. For example, if $\mathrm{R}$ parity is broken, it would be possible to probe SUSY even at the LEP1 energies through genuinely new signatures, such as single SUSY particle production [121]! Therefore one needs to re-analyse the existing data in order to place limits on these models. We now turn to a discussion of explicit and spontaneous breaking of $\mathrm{R}$ parity.

\subsection{Explicit R Parity Violation}

The minimal supersymmetric extension of the $S U(2) \otimes U(1)$ theory in general violates lepton and baryon number conservation. Indeed, $S U(2) \otimes U(1)$ gauge invariance and SUSY are consistent 
with adding to the basic superpotential, eq. (92), many Yukawa terms that violate lepton number conservation, such as

$$
W_{R}=\varepsilon_{a b}\left[\lambda_{i j k} \hat{L}_{i}^{a} \hat{L}_{j}^{b} \hat{E}_{k}^{C}+\lambda_{i j k}^{\prime} \hat{L}_{i}^{a} \hat{Q}_{j}^{b} \hat{D}_{k}^{C}+\epsilon_{i} \hat{L}_{i}^{a} \hat{H}_{u}^{b}\right]
$$

Here $\mathrm{i}, \mathrm{j}, \mathrm{k}$ denote flavour indices and $\lambda$ is anti-symmetric in $\mathrm{i}, \mathrm{j}$. Similarly, one could add terms such as $\hat{U}^{c} \hat{D}^{c} \hat{D}^{c}$, as they are consistent with all symmetries of the Standard Model, plus supersymmetry. The presence of such terms, along with those in eq. (102), will lead to baryon number violating processes such as proton decay. Such terms may arise as residuals from many extended models, such as supersymmetric GUTS [122]. In their presence R parity symmetry is broken explicitly, as can easily be checked. The bilinear in $W_{R}$ parametrizes effectively, for many purposes, the main effects of theories with spontaneous breaking. Moreover, as we will see below, it plays a very important role in the physics of the Higgs sector.

There are several constraints on these couplings, some of which are quite stringent [123]. Recently the Aleph collaboration has placed limits on explicitly broken R-parity models by considering the pair production of the lightest neutralino followed by its decay as would be induced under the very restrictive assumption that a single of the $\hat{L} \hat{L} \hat{E}^{c}$ coupling is present [124]. Other couplings are very much constrained. For example, the presence of $\hat{U}^{c} \hat{D}^{c} \hat{D}^{c}$ terms, along with those in eq. (102), will lead to unacceptably fast proton decay. They may also be constrained by the requirement of non-erasure of a primordial baryon asymmetry [125]. Therefore one normally forbids these terms by hand, invoking $\mathrm{R}$ parity conservation. It is possible, however, that $R$ parity is explicitly broken only by a subset of these terms, at a sizeable level, yet fully consistent with observation. The missing terms could arise by imposing some global and/or discrete symmetry. Moreover, explicit $R_{p}$ violating interactions could be tolerated in the presence of a mechanism that could generate a nonzero baryon asymmetry at low energy, as suggested in ref. [126].

What is least pleasant of models with explicitly broken $\mathrm{R}$ parity is that they involve too large a number of arbitrary parameters in the Yukawa sector, which limits considerably their predictive power and the ability to map out systematically their possible effects at accelerators. For this reason, we will focus our discussion mostly on the case of Spontaneous R Parity Violation.

\subsection{Spontaneous R Parity Violation}

In Spontaneous R Parity Violation scenarios the breaking of R-parity is driven by right-handed isosinglet sneutrino vacuum expectation values (VEVS) [127, 128, so that the associated Goldstone boson (Majoron) is mostly singlet. As a result the $Z$ does not decay by Majoron emission, in agreement with LEP observations [3].

Here we focus on what is the conceptually simplest model for Spontaneous R Parity Violation, 
in which two $S U(2) \otimes U(1)$ singlet leptons, instead of one, are added in each family 127 . The conceptual simplicity of the model follows from the fact that the magnitude of all R Parity violating effects is strictly correlated to the mass of the tau neutrino. Although most of the subsequent discussion applies equally well to models where the Majoron is absent, due to an enlarged gauge structure [129, 130], or to other $S U(2) \otimes U(1)$ models with spontaneously broken $\mathrm{R}$ parity [128, for definiteness we will focus on the simplest model and start by recalling its main ingredients. Indeed, many of the phenomenological features relevant for the accelerator studies already emerge in an effective model where the spontaneous violation of $\mathrm{R}$ parity is reproduced through a the addition of the explicit bilinear superpotential term in eq. (102) 131.

The superpotential is given by

$$
h_{u} Q H_{u} U^{c}+h_{d} H_{d} Q D^{c}+h_{e} \ell H_{d} E^{c}+\left(h_{0} H_{u} H_{d}-\epsilon^{2}\right) \Phi+h_{\nu} \ell H_{u} \nu^{c}+h \Phi S \nu^{c}+\text { h.c. }
$$

where we have omitted the hats in the superfields, as well as generation space indices in the coupling matrices $h_{u}, h_{d}, h_{e}, h_{\nu}, h$. This superpotential conserves total lepton number and $\mathrm{R}$ parity. The superfields $\left(\Phi, \nu^{c}{ }_{i}, S_{i}\right)$ are singlets under $S U(2) \otimes U(1)$ and carry a conserved lepton number assigned as $(0,-1,1)$, respectively. These additional singlets $\nu^{c}, S$ [20] and $\Phi$ [113 may drive the spontaneous violation of $\mathrm{R}$ parity in the model [127]. This leads to the existence of a Majoron given by the imaginary part of

$$
\frac{v_{L}^{2}}{V v^{2}}\left(v_{u} H_{u}-v_{d} H_{d}\right)+\frac{v_{L}}{V} \tilde{\nu_{\tau}}-\frac{v_{R}}{V}{\tilde{\nu^{c}}}_{\tau}+\frac{v_{S}}{V} \tilde{S}_{\tau}
$$

where the isosinglet VEVS

$$
v_{R}=\left\langle\tilde{\nu}_{R \tau}\right\rangle, \quad v_{S}=\left\langle\tilde{S}_{\tau}\right\rangle
$$

with $V=\sqrt{v_{R}^{2}+v_{S}^{2}}$ characterize R-parity or lepton number breaking and the isodoublet VEVS

$$
v_{u}=\left\langle H_{u}\right\rangle, \quad v_{d}=\left\langle H_{d}\right\rangle
$$

drive electroweak breaking and the fermion masses. The combination $v^{2}=v_{u}^{2}+v_{d}^{2}+v_{L}^{2}$ is fixed by the W,Z masses. Finally, there is a small seed of $\mathrm{R}$ parity breaking in the doublet sector, i.e.

$$
v_{L}=\left\langle\tilde{\nu}_{L \tau}\right\rangle
$$

whose magnitude is now related to the Yukawa coupling $h_{\nu}$. Since this vanishes as $h_{\nu} \rightarrow 0$, we can naturally obey the limits from stellar energy loss [132.

For our subsequent discussion we need the chargino and neutralino mass matrices. The form of the chargino mass matrix is common to a wide class of $S U(2) \otimes U(1)$ SUSY models with

\footnotetext{
${ }^{i}$ One may add just a single pair of singlet lepton superfields, instead of three.
} 
spontaneously broken $\mathrm{R}$ parity. It is given by

\begin{tabular}{c|ccc} 
& $e_{j}^{+}$ & $\tilde{H}_{u}^{+}$ & $-i \tilde{W}^{+}$ \\
\hline$e_{i}$ & $h_{e i j} v_{d}$ & $-h_{\nu i j} v_{R j}$ & $\sqrt{2} g_{2} v_{L i}$ \\
$\tilde{H}_{d}^{-}$ & $-h_{e i j} v_{L i}$ & $\mu$ & $\sqrt{2} g_{2} v_{d}$ \\
$-i \tilde{W}^{-}$ & 0 & $\sqrt{2} g_{2} v_{u}$ & $M_{2}$
\end{tabular}

Two matrices $\mathrm{U}$ and $\mathrm{V}$ are needed to diagonalize the $5 \times 5$ (non-symmetric) chargino mass matrix

$$
\begin{aligned}
& \chi_{i}^{+}=V_{i j} \psi_{j}^{+} \\
& \chi_{i}^{-}=U_{i j} \psi_{j}^{-}
\end{aligned}
$$

where the indices $i$ and $j$ run from 1 to 5 and $\psi_{j}^{+}=\left(e_{1}^{+}, e_{2}^{+}, e_{3}^{+}, \tilde{H}_{u}^{+},-i \tilde{W}^{+}\right)$and $\psi_{j}^{-}=$ $\left(e_{1}^{-}, e_{2}^{-}, e_{3}^{-}, \tilde{H}_{d}^{-},-i \tilde{W}^{-}\right)$.

Under reasonable approximations, we can truncate the neutralino mass matrix so as to obtain an effective $7 \times 7$ matrix of the following form 127

\begin{tabular}{c|ccccc} 
& $\nu_{i}$ & $\tilde{H}_{u}$ & $\tilde{H}_{d}$ & $-i \tilde{W}_{3}$ & $-i \tilde{B}$ \\
\hline$\nu_{i}$ & 0 & $h_{\nu i j} v_{R j}$ & 0 & $g_{2} v_{L i}$ & $-g_{1} v_{L i}$ \\
$\tilde{H}_{u}$ & $h_{\nu i j} v_{R j}$ & 0 & $-\mu$ & $-g_{2} v_{u}$ & $g_{1} v_{u}$ \\
$\tilde{H}_{d}$ & 0 & $-\mu$ & 0 & $g_{2} v_{d}$ & $-g_{1} v_{d}$ \\
$-i \tilde{W}_{3}$ & $g_{2} v_{L i}$ & $-g_{2} v_{u}$ & $g_{2} v_{d}$ & $M_{2}$ & 0 \\
$-i \tilde{B}$ & $-g_{1} v_{L i}$ & $g_{1} v_{u}$ & $-g_{1} v_{d}$ & 0 & $M_{1}$
\end{tabular}

This matrix is diagonalized by a $7 \times 7$ unitary matrix $\mathrm{N}$,

$$
\chi_{i}^{0}=N_{i j} \psi_{j}^{0}
$$

where $\psi_{j}^{0}=\left(\nu_{i}, \tilde{H}_{u}, \tilde{H}_{d},-i \tilde{W}_{3},-i \tilde{B}\right)$, with $\nu_{i}$ denoting weak-eigenstate neutrinos (the indices $i$ and $j$ run from 1 to 7 ).

Here we make the same parameter assumptions and conventions as used in the MSSM. Typical values for the SUSY parameters $\mu$ and $M_{2}$ are as before. The parameters $h_{\nu i, 3}$ lie in the range given by

$$
10^{-10} \leq h_{\nu 13}, h_{\nu 23} \leq 10^{-1} \quad 10^{-5} \leq h_{\nu 33} \leq 10^{-1}
$$


while the expectation values are chosen as

$$
\begin{array}{ll}
v_{L}=v_{L 3}=100 \mathrm{MeV} & v_{L 1}=v_{L 2}=0 \\
v_{R}=v_{R 3}=1000 \mathrm{GeV} & v_{R 1}=v_{R 2}=0 \\
v_{S}=1000 \mathrm{GeV} & 1 \lesssim \tan \beta=\frac{v_{u}}{v_{d}} \lesssim \frac{m_{t}}{m_{b}}
\end{array}
$$

The diagonalization of eq. (111) gives rise to the mixing of the neutralinos with the neutrinos, leading to R-parity violating gauge couplings and to neutrino masses, mainly the $\nu_{\tau}$ mass. Although the $\nu_{\tau}$ can be quite massive, it is perfectly consistent with cosmology [52], including primordial nucleosynthesis, as it can both decay through Majoron emission $\nu_{\tau} \rightarrow \nu_{\mu}+J$ [56, 57] due to flavour non-diagonal couplings such as $h_{\nu 23}$, as well as annihilate to a Majoron pair due to the diagonal coupling $h_{\nu 33}$ 63. Both processes can be quite efficient cosmologically in order to bypass the required restrictions, leaving the $\nu_{\tau}$ mass free to attain its maximum valued allowed by laboratory experiments. On the other hand, the tiny $\nu_{e}$ and $\nu_{\mu}$ masses may be chosen to lie in the range where resonant $\nu_{e} \rightarrow \nu_{\mu}$ conversions provide an explanation of solar neutrino deficit. Due to this peculiar hierarchical pattern, one can regard the associated $\mathrm{R}$ parity violating processes as a tool to probe the physics underlying the solar neutrino conversions in this model [57]. Indeed, the rates for such rare decays can be used to discriminate between large and small mixing angle MSW solutions to the solar neutrino problem [76]. Typically, in the small mixing region can have larger rare decay branching ratios than in the large mixing region, as seen in Figure 5 of ref. [57].

As already mentioned, the $\nu_{\tau}$ mass shows a direct correlation with the magnitude of R-parity violating phenomena, making this model a especially useful way to parametrize the resulting physics.

Using the above diagonalizing matrices $\mathrm{U}, \mathrm{V}$ and $\mathrm{N}$ one can write the electroweak currents of the mass-eigenstate fermions. For example, the charged current Lagrangian describing the weak interaction between charged lepton/chargino and neutrino/neutralinos may be written as

$$
\frac{g}{\sqrt{2}} W_{\mu} \bar{\chi}_{i}^{-} \gamma^{\mu}\left(K_{L i k} P_{L}+K_{R i k} P_{R}\right) \chi_{k}^{0}+H . C .
$$

where $P_{L, R}$ are the two chiral projectors and the $5 \times 7$ coupling matrices $K_{L, R}$ may be written as

$$
\begin{array}{r}
K_{L i k}=\eta_{i}\left(-\sqrt{2} U_{i 5} N_{k 6}-U_{i 4} N_{k 5}-\sum_{m=1}^{3} U_{i m} N_{k m}\right) \\
K_{R i k}=\epsilon_{k}\left(-\sqrt{2} V_{i 5} N_{k 6}+V_{i 4} N_{k 4}\right)
\end{array}
$$

The matrix $K_{L i k}$ is the analogous of the matrix $K$ introduced in ref [6]. These couplings break R-parity for $i=1 . .3$ and $k=4 . .7$, and $i=4,5$ and $k=1 . .3$. 
Similarly, the corresponding neutral current Lagrangian describing the weak interaction of the charged lepton and charginos, as well as the neutrinos and neutralinos may be written as

$$
\frac{g}{\cos \theta_{W}} Z_{\mu}\left\{\bar{\chi}_{i}^{-} \gamma^{\mu}\left(\eta_{i} \eta_{k} O_{L i k}^{\prime} P_{L}+O_{R i k}^{\prime} P_{R}\right) \chi_{k}^{-}+\frac{1}{2} \bar{\chi}_{i}^{0} \gamma^{\mu}\left(\epsilon_{i} \epsilon_{k} O_{L i k}^{\prime \prime} P_{L}+O_{R i k}^{\prime \prime} P_{R}\right) \chi_{k}^{0}\right\}
$$

where the $7 \times 7$ coupling matrices $O_{L, R}^{\prime}$ and $O_{L, R}^{\prime \prime}$ are given by

$$
\begin{array}{r}
O_{L i k}^{\prime}=\frac{1}{2} U_{i 4} U_{k 4}+U_{i 5} U_{k 5}+\frac{1}{2} \sum_{m=1}^{3} U_{i m} U_{k m}-\delta_{i k} \sin ^{2} \theta_{W} \\
O_{R i k}^{\prime}=\frac{1}{2} V_{i 4} V_{k 4}+V_{i 5} V_{k 5}-\delta_{i k} \sin ^{2} \theta_{W} \\
O_{L i k}^{\prime \prime}=\frac{1}{2}\left\{N_{i 4} N_{k 4}-N_{i 5} N_{k 5}-\sum_{m=1}^{3} N_{i m} N_{k m}\right\}=-O_{R i k}^{\prime \prime}
\end{array}
$$

In writing these couplings we have assumed CP conservation. Under this assumption the diagonalizing matrices can be chosen to be real. The $\eta_{i}$ and $\epsilon_{k}$ factors are sign factors, related with the relative CP parities of these fermions, that follow from the diagonalization of their mass matrices. These couplings break R-parity for $i=1 . .3$ and $k=4,5$ in the case of the charged leptons, and $i=4 . .7$ and $k=1 . .3$ for the neutral leptons.

Like all supersymmetric extensions of the Standard Model, the spontaneously broken R-parity models are constrained by data that follow from the negative searches for supersymmetric particles at LEP as well as $\bar{p} p$ collider data on gluino production. There are additional restrictions, which are more characteristic of broken R-parity models. They follow from laboratory experiments related to neutrino physics and weak interactions [15], as well as from cosmology and astrophysics. These restrictions play a very important role, as they exclude many parameter choices that are otherwise allowed by the collider constraints, while the converse is not true. The most relevant constraints come from neutrino-less double beta decay and neutrino oscillation searches, direct searches for anomalous peaks at meson decays, the limit on the tau neutrino mass, cosmological limits on the $\nu_{\tau}$ lifetime and mass, limits on muon and tau lifetimes, universality constraints, and limits on lepton flavour violating decays.

One can perform a sampling of the points which are allowed by these constraints in order to evaluate systematically the attainable value of the couplings [133]. The diagonal (R-parity conserving) couplings for the lightest neutralino and the lightest chargino are of the same order as those in the MSSM. The coupling of the lightest chargino to the $Z$ is maximum when it is mainly a gaugino. In this case $\mu \gg M_{2}$ and as a result $\left|V_{45}\right| \approx\left|U_{45}\right| \approx 1$ and $\left|V_{4 i}\right|,\left|U_{4 i}\right| \ll 1$ for $i \neq 5$. On the other hand it is minimum when it is mostly a Higgsino. In this case $\mu \ll M_{2}$ and therefore $\left|V_{44}\right| \approx\left|U_{44}\right| \approx 1$ and $\left|V_{4 i}\right|,\left|U_{4 i}\right| \ll 1$ for $i \neq 4$. Including these values in eq. (119) and eq. (120) one gets for the allowed range

$$
0.27 \lesssim\left|O_{L 44}^{\prime}\right|,\left|O_{R 44}^{\prime}\right| \lesssim 0.77
$$




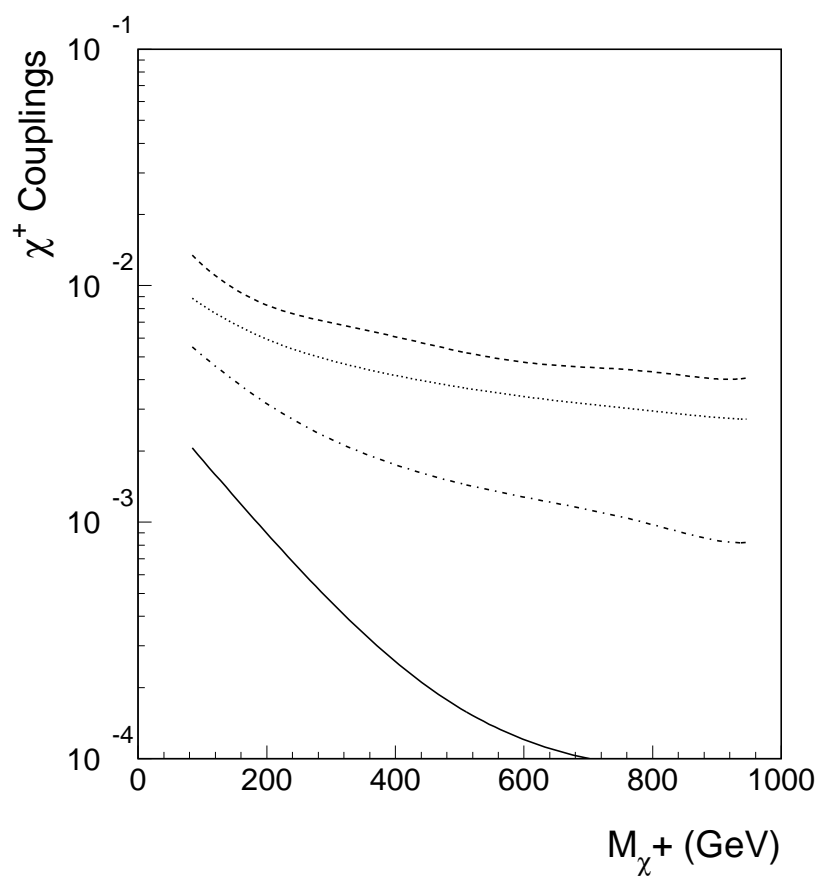

Figure 25: Presently allowed region of chargino R parity violating couplings.

From the parameters given in eq. (98), (113) and (114) and the experimental limits (specially the recent LEP limit on the lightest chargino mass) one finds that the lightest supersymmetric fermion is always a neutralino, with mass $M_{\chi^{0}} \gtrsim 30 \mathrm{GeV}$.

Similarly, the R-parity-conserving charged current couplings of the lightest chargino to the lightest neutralino after including the experimental constraints lie in the range

$$
10^{-4} \lessgtr\left|K_{L 44}\right|,\left|K_{R 44}\right| \lessgtr \frac{1}{\sqrt{2}}
$$

For the neutral current couplings of the lightest neutralino one finds, after imposing the experimental constraints

$$
\left|O_{L 44}^{\prime \prime}\right| \lesssim 0.1
$$

In what concerns the R-parity breaking couplings, the largest ones correspond to the case when the standard lepton belongs to the third family. These couplings can reach a few per cent or so for mass values accessible in accelerator studies [133]. This is illustrated in Fig. (25) Here we have considered the parameters as in eq. (98), (113) and (114) with $\tan \beta=\frac{v_{u}}{v_{d}}$ chosen to lie between 2 and 30. In Fig. (25) the various lines denote the allowed magnitudes of the left and right-handed $\mathrm{R}$ parity violating currents. The solid line denotes $\left|K_{L 43}\right|$, the dashed one $\left|K_{R 43}\right|$, the dotted one $\left|O_{L 34}^{\prime}\right|$ and, finally, the dash-dotted one denotes $\left|O_{R 34}^{\prime}\right|$.

\subsection{The Scalar Sector in the Model}

The theoretical viability of the model proposed in ref. [127 has been explicitly demonstrated and we do not plan to repeat here the detailed discussion on the minimization of the correspond- 
ing Higgs potential. Nevertheless we will make a brief summary. For simplicity we consider an effective one-generation model in which the coupling matrices $h_{\nu i j}$ and $h_{i j}$ are nonzero only for the third generation and set $h_{\nu} \equiv h_{\nu 33}$ and $h \equiv h_{33}$. We adopt the most general form for the soft SUSY breaking terms in a spontaneously broken $N=1$ super-gravity model

$$
\begin{array}{r}
V_{\text {soft }}=\tilde{m}_{0}[- \\
\left.-A h_{0} \Phi H_{u} H_{d}-B \epsilon^{2} \Phi+C h_{\nu} \tilde{\nu^{c}} \tilde{\nu} H_{u}+D h \Phi \tilde{\nu^{c}} \tilde{S}+E \hat{\mu} H_{u} H_{d}+\text { h.c. }\right] \\
+\tilde{m}_{u}^{2}\left|H_{u}\right|^{2}+\tilde{m}_{d}^{2}\left|H_{d}\right|^{2}+\tilde{m}_{L}^{2}|\tilde{\nu}|^{2}+\tilde{m}_{R}^{2}\left|\tilde{\nu^{c}}\right|^{2}+\tilde{m}_{S}^{2}|\tilde{S}|^{2}+\tilde{m}_{\Phi}^{2}|\Phi|^{2}
\end{array}
$$

We have included only the neutral scalars. These soft breaking terms have the form expected in models with minimal $N=1$ super-gravity theories characterized, at the unification scale, by universal, diagonal supersymmetry-breaking scalar masses and by trilinear scalar terms proportional to a single dimension-less parameter $A$

$$
\begin{array}{r}
C=D=A, \quad E=A-1, \quad B=A-2 \\
\tilde{m}_{u}^{2}=\tilde{m}_{d}^{2}=\tilde{m}_{L}^{2}=\tilde{m}_{R}^{2}=\tilde{m}_{S}^{2}=\tilde{m}_{\Phi}^{2}
\end{array}
$$

Since these conditions are not expected to hold at low energies, due to renormalization group evolution from the unification scale down to the electroweak scale, we allow the values of the soft breaking scalar masses to differ from their common value $\tilde{m}_{0}$ at the unification scale. We have kept however the values of $B, C, D$ and $E$ related as above and assumed, for simplicity, that all parameters in the potential are real.

With the definitions above the full scalar potential along neutral directions of the Spontaneous $\mathrm{R}$ Parity Broken model of ref. [127] is given by

$$
\begin{array}{r}
V_{\text {total }}=\left|h \Phi \tilde{S}+h_{\nu} \tilde{\nu} H_{u}\right|^{2}+\left|h_{0} \Phi H_{u}+\hat{\mu} H_{u}\right|^{2}+ \\
\left|h \Phi \tilde{\nu^{c}}\right|^{2}+\left|-h_{0} \Phi H_{d}-\hat{\mu} H_{d}+h_{\nu} \tilde{\nu} \tilde{\nu}^{c}\right|^{2}+\left|-h_{0} H_{u} H_{d}+h \tilde{\nu^{c}} \tilde{S}-\epsilon^{2}\right|^{2}+\left|h_{\nu} \tilde{\nu}^{c} H_{u}\right|^{2} \\
+\tilde{m}_{0}\left[-A\left(-h \Phi \tilde{\nu}^{c} \tilde{S}+h_{0} \Phi H_{u} H_{d}-h_{\nu} \tilde{\nu} H_{u} \tilde{\nu}^{c}\right)+(1-A) \hat{\mu} H_{u} H_{d}+(2-A) \epsilon^{2} \Phi+h . c .\right] \\
+\sum_{i} \tilde{m}_{i}^{2}\left|z_{i}\right|^{2}+\alpha\left(\left|H_{u}\right|^{2}-\left|H_{d}\right|^{2}-|\tilde{\nu}|^{2}\right)^{2}
\end{array}
$$

where $\alpha=\frac{g^{2}+g^{\prime 2}}{8}$ and $z_{i}$ denotes any neutral scalar field in the theory.

Electroweak breaking is driven by the isodoublet VEVS $v_{u}=\left\langle H_{u}\right\rangle$ and $v_{d}=\left\langle H_{d}\right\rangle$, assisted by the VEV $v_{F}$ of the scalar in the singlet superfield $\Phi$. The $\mathrm{W}$ mass is given as $m_{W}^{2} \approx \frac{g^{2}\left(v_{u}^{2}+v_{d}^{2}\right)}{2}$, while the ratio of isodoublet VEVS determines $\tan \beta=\frac{v_{u}}{v_{d}}$. This way one basically recovers the tree level $S U(2) \otimes U(1)$ spontaneous breaking scenario of ref. [113.

The spontaneous breaking of $\mathrm{R}$ parity is driven by nonzero VEVS for the scalar isosinglet neutrinos VEVS

$$
\begin{gathered}
v_{R}=\left\langle{\tilde{\nu^{c}}}_{\tau}\right\rangle \\
v_{S}=\left\langle\tilde{S_{\tau}}\right\rangle
\end{gathered}
$$


where $V=\sqrt{v_{R}^{2}+v_{S}^{2}}$ can lie anywhere in the range $\sim 10 \mathrm{GeV}-1 \mathrm{TeV}$. A necessary ingredient for the consistency of this model is the presence of a small seed of $\mathrm{R}$ parity breaking in the $S U(2)$ doublet sector,

$$
v_{L}=\left\langle\tilde{\nu}_{L \tau}\right\rangle
$$

whose typical magnitude in the model may naturally obey the astrophysical limits coming from stellar energy loss considerations from Majoron emitting processes [132].

The detailed analysis of the minimization of this potential was presented in the second paper of ref. [127]. There we have explicitly demonstrated the existence of solutions to the extremization equations following from eq. (128) which are in fact minima and not saddle points, and whose energy is lower than that of other trivial solutions where either $\mathrm{R}$ parity or electroweak symmetries are unbroken. The scale associated to the spontaneous violation of $\mathrm{R}$ parity can lie typically anywhere in the range from 10 to $1000 \mathrm{GeV}$.

The squared mass matrices of the neutral scalar bosons

$$
z_{i}=\frac{1}{\sqrt{2}}\left[\operatorname{Re}\left(z_{i}\right)+i \operatorname{Im}\left(z_{i}\right)\right]
$$

are given as

$$
M_{R i j}^{2}=\frac{1}{2}\left(\frac{\partial^{2} V}{\partial z_{i} z_{j}}+\text { c.c. }\right)+\frac{\partial^{2} V}{\partial z_{i} z_{j}^{*}}
$$

and

$$
M_{I i j}^{2}=-\frac{1}{2}\left(\frac{\partial^{2} V}{\partial z_{i} z_{j}}+\text { c.c. }\right)+\frac{\partial^{2} V}{\partial z_{i} z_{j}^{*}}
$$

They were determined at the tree level in the second paper in ref. [127] and shown to be positive-definite in large regions of parameter space. Assuming CP conservation, the real and imaginary parts do not mix, so that the mass part of the potential energy reads

$$
V_{\text {mass }}=\frac{1}{2} \operatorname{Re}\left(z_{i}\right) M_{R i j}^{2} \operatorname{Re}\left(z_{j}\right)+\frac{1}{2} \operatorname{Im}\left(z_{i}\right) M_{I i j}^{2} \operatorname{Im}\left(z_{j}\right)
$$

The $6 \times 6$ matrices obtained this way imply the existence in this model of $6 \mathrm{CP}$-even and 5 CP-odd scalars, the last ones including the massless Majoron, given by eq. (104).

Although the explicit expressions for the masses in terms of the input parameters defining the low energy theory are quite involved, a fairly simple mass formula can be derived. From eq. (133) and eq. (134) we have

$$
\operatorname{Tr} M_{R}^{2}=\operatorname{Tr} M_{I}^{2}+\sum_{i=1}^{6}\left(\frac{\partial^{2} V}{\partial z_{i} z_{j}}+\text { h.c. }\right)
$$

Using the explicit form of the potential we get that the last term in eq. (136) is just $m_{Z}^{2}$, so that

$$
\operatorname{Tr} M_{R}^{2}=\operatorname{Tr} M_{I}^{2}+m_{Z}^{2}
$$


which nicely generalizes the corresponding tree-level MSSM sum rule.

The mass spectrum for both CP-even and CP-odd scalar bosons was studied numerically in this model, both at the tree level and after including radiative corrections [134]. For centreof-mass energies attainable either at LEP200, LHC or NLC, not all of the scalar bosons are kinematically accessible. Typically one or two of the CP-even ones (h, H) will be accessible and one of the massive CP-odd (A) scalar bosons. Thus the situation becomes conceptually similar to the one considered in section 3.2.2. The main new features relevant for experimental analyses are

- the coupling of the lightest CP-even Higgs boson can be suppressed with respect to that expected in the Standard Model, as in sections 3.2.1 and 3.2.2. Indeed, it was noted in ref. [134 that in some regions of parameters of the potential the lightest CP-even Higgs boson in this model is mostly an $S U(2) \otimes U(1)$ singlet, with tiny couplings to the Z . Such a scalar boson would have escaped detection at LEP due to its small production cross section.

- the lightest CP-even Higgs bosons may decay invisibly as

$$
\begin{aligned}
& h \rightarrow J+J \\
& H \rightarrow h h \quad \text { with } \quad h \rightarrow J+J
\end{aligned}
$$

The invisible decay of the lightest CP-even Higgs boson can be quite sizeable and can therefore compete with the standard $b \bar{b}$ decay mode. This feature is similar to what we have seen in sections 3.2 .1 and 3.2 .2 .

\subsection{Implications of Spontaneous R Parity Breaking}

In the MSSM all supersymmetric particles are always produced in pairs. If $\mathrm{R}$ parity is broken, they may be singly-produced. As seen in section 4.5, in models with spontaneous $\mathrm{R}$ parity breaking the mixing of the standard leptons with the supersymmetric charginos and neutralinos leads to the existence of R-parity violating couplings in the Lagrangian when written in terms of the mass eigenstates. It is in the couplings of the $\mathrm{W}$ and the $\mathrm{Z}$ where the main $\mathrm{R}$-parity violating effects reside [133. As a result one is no longer forced to produce the SUSY particles in pairs. For example a SUSY fermion such as a chargino or a neutralino may be produced in pairs (standard MSSM production) as well as singly, in association with a $\tau$ or $\nu_{\tau}$ (R-parity breaking single production).

On the other hand the RPSUSY model rates for pair production of SUSY particles are similar to those in the MSSM. However, in contrast to the MSSM, where all supersymmetric particles 
have cascade decays finishing in the LSP which is normally a neutralino, in the RPSUSY case there are new decay channels and the supersymmetric particles can decay directly to the standard states breaking R-parity. Alternatively, they may decay through R-parity conserving cascade decays that will finish in the lightest neutralino, which then decays. This way one can generate novel supersymmetric signatures in $R$ parity violating models even when the single production SUSY particle cross sections are small.

The lightest neutralino can decay to standard states breaking R-parity. If its mass is lower than the mass of the gauge bosons these are three-body decays such as

$$
\chi^{0} \rightarrow \nu_{j} f \bar{f} \quad \chi^{0} \rightarrow l_{j} f_{u} \overline{f_{d}}
$$

where the first decays are mediated by the neutral current, while the second are charged-current mediated. Here $\mathrm{f}$ denotes any fermion, while $f_{u}$ and $f_{d}$ denote up or down-type fermions, respectively.

If the neutralino is heavier than the $\mathrm{W}$ it may have the two body decays

$$
\chi^{0} \rightarrow \ell_{j} W \quad \chi^{0} \rightarrow \nu_{j} Z
$$

The explicit expressions for the widths are given in ref. [133]. Neutralinos of mass accessible at LEP have mostly three-body decay modes mediated by charged and neutral currents. The only exception will be the two-body Majoron decay, characteristic of the simplest spontaneous $\mathrm{R}$ parity breaking models eq. (141).

In $S U(2) \otimes U(1)$ models of spontaneous breaking of R-parity the LSP is not the neutralino, but rather the Majoron, which is massless and therefore stable 1 . The existence of the Majoron implies that in $S U(2) \otimes U(1)$ spontaneously broken R-parity, the neutralino can always decay invisibly to

$$
\chi^{0} \rightarrow \nu_{j} J
$$

To the extent that this invisible decays are are important, one recovers the signal expected in the MSSM.

For definiteness let us consider the case of the lightest neutralino and chargino, which one expects could be the earliest-produced supersymmetric particles. Here are some examples of signals related to their production in the spontaneously broken R parity (RPSUSY) models:

\section{- Single chargino production in $\mathrm{Z}$ decays [121]}

$$
Z \rightarrow \chi^{ \pm} \tau^{\mp}
$$

${ }^{j}$ The Majoron may have a small mass and therefore it may decay to neutrinos and photons. However the time scales are only of cosmological interest and do not change the signal expected at the laboratory [135. 


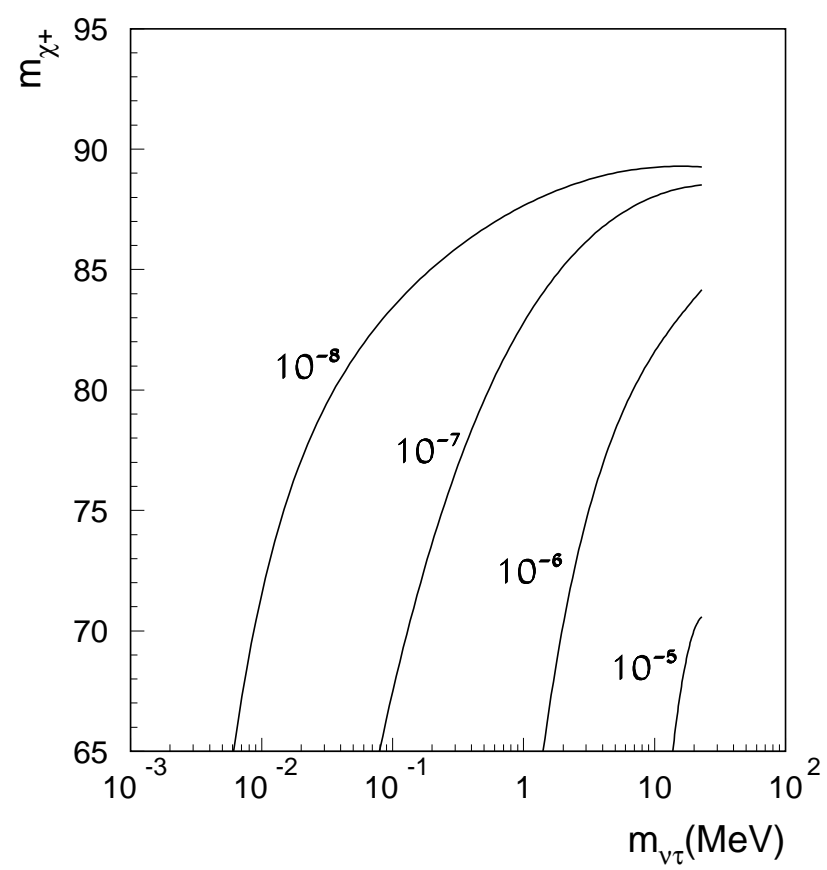

Figure 26: Allowed $Z \rightarrow \chi^{ \pm} \tau^{\mp}$ decay branching ratios in the RPSUSY model.

Table 7: Allowed branching ratios for $\mathrm{R}$ parity violating $\mathrm{Z}$ decays.

\begin{tabular}{|c|c|}
\hline \multicolumn{1}{|c|}{ channel } & strength \\
\hline$Z \rightarrow \chi^{ \pm}+\tau^{\mp}$ & $10^{-5}$ \\
$Z \rightarrow \chi^{0}+\nu_{\tau}$ & $10^{-4}$ \\
\hline
\end{tabular}

where the lightest chargino mass is assumed to be smaller than the $\mathrm{Z}$ mass. This decay is characteristic of spontaneous $\mathrm{R}$ parity violation. In the simplest models, the magnitude of $\mathrm{R}$ parity violation is correlated with the nonzero value of the $\nu_{\tau}$ mass and is restricted by a variety of experiments. Nevertheless the $\mathrm{R}$ parity violating $\mathrm{Z}$ decay branching ratios can easily exceed $10^{-6}$ (see table 7 ) and thus lie within the sensitivities of the LEP experiments performed at the $\mathrm{Z}$ pole. As illustrated in Fig. (26) the maximum branching ratio allowed by other experiments and by theory is directly correlated with $m_{\nu_{\tau}}$ which is a characteristic feature of the model of ref. 127.

\section{- Single neutralino production in $\mathrm{Z}$ decays [121]}

$$
Z \rightarrow \chi^{0} \nu_{\tau}
$$

The allowed rates for $\mathrm{R}$ parity violating $\mathrm{Z}$ decays is given in table 7 . To the extent that $\chi$ decays into charged particles are dominant the neutralino is not necessarily an origin of events with missing energy, as in the MSSM. Thus the decay $Z \rightarrow \chi^{0} \nu_{\tau}$ would give rise to 
Table 8: Allowed rates for novel decay modes in spontaneous broken $\mathrm{R}$ parity.

\begin{tabular}{|c|c|}
\hline channel & strength \\
\hline$Z \rightarrow \gamma+J$ & $10^{-5}$ \\
$\tau \rightarrow \mu+J$ & $10^{-3}$ \\
$\tau \rightarrow e+J$ & $10^{-4}$ \\
\hline
\end{tabular}

zen events, similar to those of the MSSM, but where the missing energy is carried by the $\nu_{\tau}$ and the visible tracks come from the decays of the $\chi$. The searches for single particle SUSY production at LEP1 should place restrictions on the parameter space available for studies at LEP200 energies [136].

- Pair lightest neutralino production in $\mathrm{Z}$ decays [121], followed by neutralino decays

Even if its single production cross section is small, the $\chi \chi$ pair production process at LEP will generate zen events where one $\chi$ decays visibly and the other invisibly. The corresponding zen-event rates can therefore be larger than in the MSSM and may occur even if there is no energy to produce the next-to-lightest neutralino $\chi^{\prime}$.

\section{- Majoron emitting decays}

Another possible signal of the RPSUSY models based on the simplest $S U(2) \otimes U(1)$ gauge group is rare decays of muons and taus and $\mathrm{Z}$ bosons involving Majoron emission. One expects possibly large rates for this new class of decays, since in these models the characteristic lepton number breaking scale is similar to the weak scale. The allowed branching ratios are illustrated in table 8. For example the LFV decays with single Majoron emission in $\mu$ and $\tau$ decays would be "seen" as bumps in the final lepton energy spectrum, at half of the parent lepton mass in its rest frame. On the other hand, the $Z \rightarrow \gamma+J$ decay would give rise to monochromatic photons 137. As an illustration of the $\nu_{\tau}$ mass dependence of the allowed decay branching ratios see Figure 2 given in ref. 137.

The allowed rates for single Majoron emitting $\mu$ and $\tau$ decays have been determined in ref. [138 and are also shown in table 3 to be compatible with present experimental sensitivities [5]. As an illustration of the $\nu_{\tau}$ mass dependence of the allowed decay branching ratios see Figures given in ref. [138. This example also illustrates how the search for rare decays can be a more sensitive probe of neutrino properties than the more direct searches for neutrino masses, and 


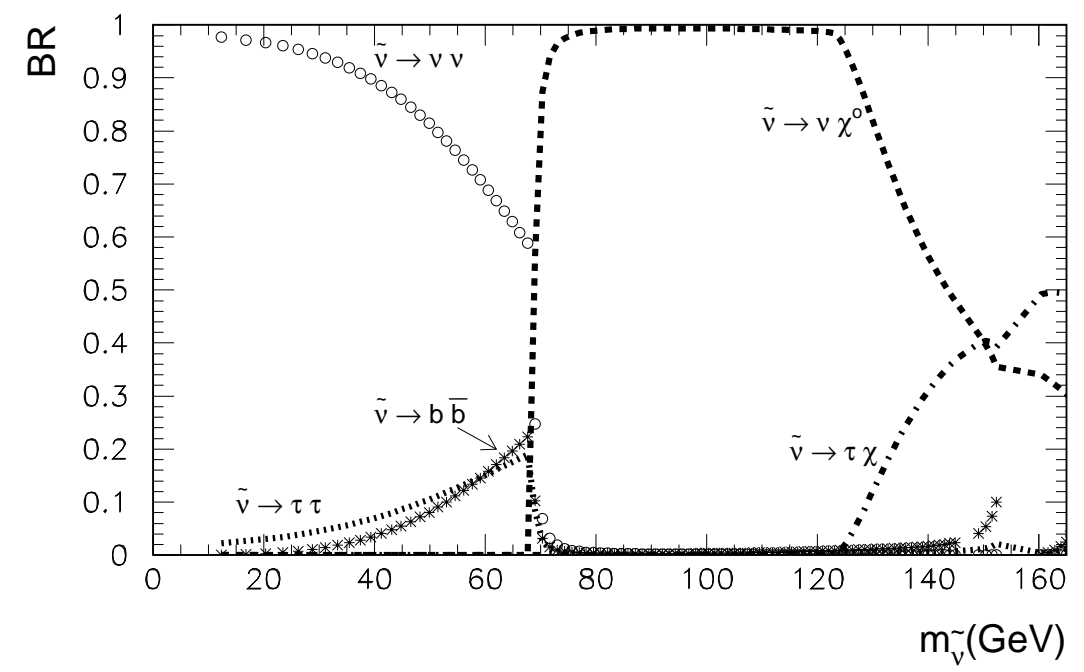

Figure 27: Sneutrino decay branching ratios in the RPSUSY model.

therefore complementary. Moreover, they are ideally studied at a tau-charm factory [89, 35].

\subsection{R Parity Breaking Scalar Boson Decays}

Explicit violation of $R$ parity in the minimal supersymmetric model through bilinear terms $\hat{L} \hat{H}_{u}$ plays an important role in the scalar sector [131]. The presence of such bilinear superpotential term will induce the mixing of sleptons with Higgs bosons, thus affecting the decays of both.

The most illustrative example of this is the possibility that, below the threshold for SUSY particle production, the sneutrino mostly decays to Standard Model particles, as shown in Fig. (27) However, even when the sneutrino is not the lightest SUSY particle, there may be a sizeable branching ratio for the $\mathrm{R}$ parity violating sneutrino decays, even for a moderately small value of the Higgsino-lepton superpotential mixing parameters $\epsilon_{i}$.

As shown in ref. [131 this may lead also to sizeable branching ratio for the supersymmetric Higgs boson decay mode $H \rightarrow \chi \ell$, where $\chi$ denotes the lightest supersymmetric particle - LSP - or a chargino, and $\ell$ is either a neutrino or a tau lepton. This $\mathrm{R}$ parity violating Higgs boson decay mode may compete favourably with the conventional decay $H \rightarrow b \bar{b}$, at least for some ranges of parameters of the model. In these estimates one has taken into account the relevant constraints on $R$ parity violation, as well as those coming from SUSY particle searches.

\section{Outlook}

In these lectures we have covered two main areas in electroweak physics where the Standard Model is most likely an incomplete description of Nature. As a first subject we covered neutrino 
physics. Besides being suggested by theory, neutrino masses seem to be required to fit present astrophysical and cosmological observations related to solar and atmospheric neutrinos, as well as the data on primordial density inhomogeneities on a variety of scales in the Universe, in addition to some inconclusive hints from the LSND experiment at Los Alamos.

If they have non-standard properties, such as mass, neutrinos could be responsible for a wide variety of laboratory implications. These new phenomena would cover an impressive range of energies, from nuclear $\beta$ and double $\beta$ decays, especially neutrino-less, now searched with greater sensitivity with enriched germanium. Such experiments could probe the quasidegenerate neutrino scenario for the joint explanation of hot dark matter, solar and atmospheric neutrino anomalies. Moving to neutrino oscillations, soon one will probe larger regions of $\nu_{\mu} \rightarrow$ $\nu_{\tau}$ (and, as a result, also improve on $\nu_{e} \rightarrow \nu_{\tau}$ ) oscillation parameters at CERN and Fermilab accelerator experiments. The future long-baseline experiments will settle the issue raised by present atmospheric neutrino data. On the other hand SuperKamiokande should start operating soon, while we still wait for the important Borexino and Sudbury experiments to shed further light on the solar neutrino issue. Finally, a new generation of experiments capable of more accurately measuring the cosmological temperature anisotropies at smaller angular scales than COBE, would test different models of structure formation, and presumably clarify the role of neutrinos as dark matter. We have illustrated how neutrinos could imply rare processes with lepton flavour violation, as well as new signatures at high energy accelerators, such as LEP. Such experiments are complementary to those at low energies and can also indirectly test neutrino properties in an important way.

Moving to the electroweak symmetry breaking sector, we saw how the Standard Model Higgs boson mass can be tested through precision electroweak data, with the most recent LEP and SLC electroweak results plus Tevatron top-quark mass measurement giving a weak preference for a light Higgs boson mass of order $m_{Z}$. We discussed the theoretical restrictions on the Higgs boson mass from vacuum stability and from requiring that the Standard Model picture of electroweak symmetry breaking holds up to a given cutoff scale $\Lambda$ and argued that the Standard Model by itself cannot be a fundamental theory of particle interactions. It must break down once the energy is raised beyond some critical value of $\Lambda$, unknown at present. This value could lie anywhere between a few hundred $\mathrm{GeV}$ up to the Planck scale. If supersymmetry is the new physics then we should identify the scale $\Lambda$ at which the Standard Model breaks down as the low-energy supersymmetry breaking scale, which should be chosen to lie between $m_{Z}$ and about $1 \mathrm{TeV}$, so that supersymmetry can solve the hierarchy problem. Finally, we summarized the status of the present searches for the Higgs boson in the Standard Model and in the MSSM, as well as the prospects for LEP200 and LHC.

We discussed how neutrino physics may unexpectedly and quite substantially affect the prop- 
erties of the electroweak symmetry breaking sector. For example, in models where the neutrino masses are generated due to the spontaneous violation of lepton number at the weak scale the main mode of Higgs boson decay can be into the invisible Majorons, leading to a missing momentum signature. There are already interesting limits on this class of models that follow from the past runs of the LEP experiments at the $\mathrm{Z}$ pole. We have also discussed the prospects for invisibly decaying Higgs boson searches at LEP 200, both in the "Higgs-strahlung" $e^{+} e^{-} \rightarrow H Z$ channel, as well as in the associated production channel $e^{+} e^{-} \rightarrow H A$.

Finally we reviewed the basic minimal supersymmetric Standard Model phenomenology, as well as the extensions with $\mathrm{R}$ parity breaking. These would allow the single production of SUSY particles as well as the decay of the lightest SUSY particle. We have discussed some of the novel signatures that would be associated to such models with $\mathrm{R}$ parity violation, especially in the decays of $\mathrm{Z}$ bosons, Higgs bosons, sleptons, muons and $\tau$ leptons.

\section{Acknowledgements}

This paper has been supported by DGICYT under Grant number PB92-0084. I thank the organizers for the hospitality and for arranging a very pleasant school. My gratitude to all my collaborators, for the fun and for their important contribution to the work reported here. Thanks are also due to Marcela Carena, Daniel Denegri, Paul Langacker, Rocky Kolb, Doug Norman and Atsuto Suzuki, who provided me with some of the figures. I thank Jorge Romão and Hiroshi Nunokawa for comments on the manuscript.

\section{References}

[1] See, for example, P. W. Higgs, Phys. Lett. 12 (1964) 132; Phys. Rev. Lett. 13 (1964) 508.

[2] R. Mohapatra, P. Pal, Massive Neutrinos in Physics and Astrophysics, World Scientific (1991); M. Fukugita, T. Yanagida, in Physics and Astrophysics of Neutrinos, Springer (1994); C. W. Kim, A. Pevsner, Neutrinos in Physics and Astrophysics, Harwood (1993)

[3] M. Martinez, in proceedings of Int. Workshop on Elementary Particle Physics: Present and Future, ed. A. Ferrer and J. W. F. Valle, World Scientific, in press.

[4] CDF collaboration, Phys. Rev. D52 (1995) 2609; Phys. Rev. Lett. 74 (1995) 2626; D0 collaboration, Phys. Rev. D52 (1995) 4877; Phys. Rev. Lett. 74 (1995) 2632; see also J. M. Benlloch, in Elementary Particle Physics: Present and Future, ed. A. Ferrer and J. W. F. Valle, (World Scientific, 1996) in press. 
[5] L. Montanet et al Phys. Rev. D50 (1994) 1173 and 1995 off-year update for the 1996 edition (URL: http://pdg.lbl.gov/).

[6] J. Schechter and J. W. F. Valle, Phys. Rev. D22 (1980) 2227

[7] J. Schechter and J. W. F. Valle, Phys. Rev. D21 (1980) 309

[8] M. Kobayashi, T. Maskawa, Prog. Theor. Phys. 49 (1973) 652

[9] L. Maiani, Phys. Lett. B62 (1976) 183

[10] L. Wolfenstein, Phys. Rev. Lett. 51 (1983) 1945

[11] S. Glashow, J. Iliopoulos, L. Maiani, Phys. Rev. D2 (1970) 1285.

[12] K. M. Case, Phys. Rev. 107 (1957) 307.

[13] J. Schechter, J. W. F. Valle, Phys. Rev. D24 (1981) 1883, Phys. Rev. D25 (1982) 283.

[14] L. Wolfenstein, Phys. Lett. B107 (1981) 77

[15] For recent reviews see J. W. F. Valle, Gauge Theories and the Physics of Neutrino Mass, Prog. Part. Nucl. Phys. 26 (1991) 91-171 (ed. A. Faessler); and G. Gelmini and S. Roulet, UCLA/94/TEP/36 and references therein.

[16] R.N. Mohapatra and G. Senjanovic, Phys. Rev. D23 (1981) 165; Phys. Rev. Lett. 44 (1980) 912, and references therein.

[17] M. Gell-Mann, P. Ramond, R. Slansky, in Supergravity, ed. D. Freedman, P. van Nieuwenhuizen (North Holland, 1979); T. Yanagida, in Proceedings of the Workshop on the Baryon Number of the Universe and Unified Theories, ed. O. Sawada and A. Sugamoto, KEK report $79-18,1979$.

[18] A. Yu. Smirnov, Phys. Rev. D48 (1994) 3264

[19] D. Wyler, L. Wolfenstein, Nucl. Phys. B218 (1983) 205

[20] R. Mohapatra, J. W. F. Valle, Phys. Rev. D34 (1986) 1642; J. W. F. Valle, Nucl. Phys. B (Proc. Suppl.) B11 (1989) 118-177

[21] J. Bernabeu, A. Santamaria, J. Vidal, A. Mendez, J. W. F. Valle, Phys. Lett. B187 (1987) 303; J. G. Korner, A. Pilaftsis, K. Schilcher, Phys. Lett. B300 (1993) 381

[22] G. C. Branco, M. N. Rebelo, J. W. F. Valle, Phys. Lett. B225 (1989) 385; N. Rius, J. W. F. Valle, Phys. Lett. B246 (1990) 249 
[23] M. Dittmar, M. C. Gonzalez-Garcia, A. Santamaria, J. W. F. Valle, Nucl. Phys. B332 (1990) 1; M. C. Gonzalez-Garcia, A. Santamaria, J. W. F. Valle, ibid. B342 (1990) 108.

[24] M. C. Gonzalez-Garcia, J. W. F. Valle, Phys. Lett. B216 (1989) 360.

[25] Y. Chikashige, R. Mohapatra, R. Peccei, Phys. Rev. Lett. 45 (1980) 1926

[26] A. Joshipura and J. W. F. Valle, Nucl. Phys. B397 (1993) 105; J. C. Romão, F. de Campos, and J. W. F. Valle, Phys. Lett. B292 (1992) 329. A. S. Joshipura, S. Rindani, Phys. Rev. Lett. 69 (1992) 3269; R. Barbieri, and L. Hall, Nucl. Phys. B364 (1991) 27; G. Jungman and M. Luty, Nucl. Phys. B361 (1991) 24; E. D. Carlson and L. B. Hall, Phys. Rev. D40 (1989) 3187; S. Bertolini, A. Santamaria, Phys. Lett. B213 (1988) 487

[27] A. Zee, Phys. Lett. B93 (1980) 389;

[28] K. S. Babu, Phys. Lett. B203 (1988) 132

[29] P. Langacker, D. London, Phys. Rev. D38 (1988) 907; E. Nardi, E. Roulet, D. Tommasini, Phys. Lett. B344 (1995) 225

[30] C. Lacasta, Doctoral Thesis, Valencia University, 1995; J. Fuster et al, paper in preparation. See also OPAL collaboration, Phys. Lett. B247 (1990) 448 and Phys. Lett. B295 (1992) 371

[31] J. Schechter, J. W. F. Valle, Phys. Rev. D23 (1981) 1666. S. M. Bilenky, J. Hosek, S. Petcov, Phys. Lett. B94 (1980) 495; I. Kobzarev, et al., Sov. J. Nucl. Phys. 32 (1980) 1590; M. Doi, et al., Phys. Lett. B102 (1981) 323

[32] M. Dittmar, J. W. F. Valle, contribution to the High Luminosity at LEP working group, yellow report CERN-91/02, p. 98-103, Fig. 3.22 and 3.23

[33] ALEPH collaboration, Phys. Lett. B334 (1994) 244

[34] M. C. Gonzalez-Garcia, J. W. F. Valle, Mod. Phys. Lett. A7 (1992) 477; erratum Mod. Phys. Lett. A9 (1994) 2569

[35] R. Alemany et. al. hep-ph/9307252, published in ECFA study group report for a B Meson factory, ed. R. Aleksan, A. Ali, p. 191-211

[36] A. Ilakovac, A. Pilaftsis, Nucl. Phys. B437 (1995) 491; A. Pilaftsis, Mod. Phys. Lett. A9 (1994) 3595

[37] OPAL collaboration, Phys. Lett. B254 (1991) 293 and Zeit. fur Physik C67 (1995) 365; L3 collaboration, Phys. Rep. 236 (1993) 1-146; Phys. Lett. B316 (1993) 427, DELPHI collaboration, Phys. Lett. B359 (1995) 411. 
[38] L. Hall, V. Kostelecky, S. Raby, Nucl. Phys. B267 (1986) 415

[39] F. Borzumati, A. Masiero, Phys. Rev. Lett. 57 (1986) 961; T. Kosmas, G. Leontaris, J. Vergados Phys. Lett. B219 (1989) 457; A. Gabbiani, A. Masiero, Nucl. Phys. B322 (1989) 235

[40] R. Barbieri, L. Hall, A. Strumia, Nucl. Phys. B445 (1995) 219

[41] V. Lobashev, First Results of the Troitsk Experiment on the Search for Electron Neutrino Rest Mass in Tritium Decay, preprint INR 862/94, 1994

[42] D. Buskulic et al., Phys. Lett. B349 (1995) 585.

[43] J. Gómez-Cadenas, M. C. González-García, Phys. Rev. D39 (1989) 1370; J. GómezCadenas et al., Phys. Rev. D41 (1990) 2179

[44] See e.g. J. Schneps, Nucl. Phys. B (Proc. Suppl.) 31 (1993) 307.

[45] J. Gómez-Cadenas, M. C. González-García, hep-ph/9504246

[46] J. Hill, Phys. Rev. Lett. 75 (1995) 2654

[47] CHORUS and NOMAD proposals CERN-SPSLC/91-21 (1992) and CERN-SPSC/90-42 (1992); K. Kodama et al., FNAL proposal P803 (1991).

[48] J. Schechter and J. W. F. Valle, Phys. Rev. D25 (1982) 2951

[49] H. Klapdor, Prog. Part. Nucl. Phys. 32 (1994) 261.

[50] W. Haxton, Nucl. Phys. B (Proc. Suppl.) 31 (1993) 88.

[51] L. Wolfenstein, Nucl. Phys. B186 (1981) 147; J. W. F. Valle, Phys. Rev. D27 (1983) 1672 and references therein

[52] E. Kolb, M. Turner, The Early Universe, Addison-Wesley, 1990, and references therein.

[53] J. Bardeen, J. Bond and G. Efstathiou, Astrophys. J. 321 (1987) 28; J. Bond and G. Efstathiou, Phys. Lett. B265 (1991) 245; M. Davis et al., Nature 356 (1992) 489; S. Dodelson, G. Gyuk and M. Turner, Phys. Rev. Lett. 72 (1994) 3754; H. Kikuchi and E. Ma, Phys. Rev. D51 (1995) 296; H. B. Kim and J. E. Kim, Nucl. Phys. B433 (1995) 421; M. White, G. Gelmini and J. Silk, Phys. Rev. D51 (1995) 2669; A. S. Joshipura and J. W. F. Valle, Nucl. Phys. B440 (1995) 647.

[54] A.D. Dolgov, proceedings of TAUP95, Toledo.

[55] J. Schechter, J. W. F. Valle, Phys. Rev. D25 (1982) 774 
[56] J. W. F. Valle, Phys. Lett. B131 (1983) 87; G. Gelmini, J. W. F. Valle, Phys. Lett. B142 (1984) 181; J. W. F. Valle, Phys. Lett. B159 (1985) 49; M. C. Gonzalez-Garcia, J. W. F. Valle, Phys. Lett. B216 (1989) 360 A. Joshipura, S. Rindani, Phys. Rev. D46 (1992) 3000; for an early discussion see J. Schechter and J. W. F. Valle, Phys. Rev. D25 (1982) 774

[57] J. C. Romão and J. W. F. Valle. Phys. Lett. B272 (1991) 436; Nucl. Phys. B381 (1992) 87.

[58] N. Hata, et al Phys. Rev. Lett. 75 (1995) 3977; C. J. Copi, D. N. Schramm and M. S. Turner, Science 267 (1995) 192.

[59] For a recent review see S. Sarkar, Oxford preprint OUTP-95-16P

[60] E.W. Kolb, M.S. Turner, A. Chakravorty and D.N. Schramm, Phys. Rev. Lett. 67 (1991) 533.

[61] A.D. Dolgov and I.Z. Rothstein, Phys. Rev. Lett. 71 (1993) 476.

[62] M. Kawasaki, G. Steigman and H.-S. Kang, Nucl. Phys. B402 (1993) 323, B419 (1994) 105; S. Dodelson, G. Gyuk and M.S. Turner, Phys. Rev. D49 (1994) 5068

[63] A.D. Dolgov, S. Pastor, J.C. Romão and J.W.F. Valle, in preparation.

[64] A. Joshipura and J. W. F. Valle, Nucl. Phys. B397 (1993) 105; see also ref. [127 and 128

[65] C. Athanassopoulos et al, Phys. Rev. Lett. 75 (1995) 2650; D. Caldwell, talk at ICHEP94, Glasgow, 1994, preprint UCSB-HEP-95-03

[66] G. F. Smoot et al., Astrophys. J. 396 (1992) L1-L5; E.L. Wright et al., Astrophys. J. 396 (1992) L13

[67] N. Vittorio, talk at TAUP95, Toledo, Sept. 1995; M. Davis, F.J. Summers, and D. Schagel, Nature 359 (1992) 393; A.N. Taylor and M. Rowan-Robinson, ibid. 359 (1992) 396; R.K. Schaefer and Q. Shafi, Nature 359 (1992) 199; J.A. Holtzman and J.R. Primack, Astrophys. J. 405 (1993) 428; A. Klypin et al., Astrophys. J. 416 (1993) 1

[68] R. Rowan-Robinson, in Cosmological Dark Matter, (World Scientific, 1994), ed. J. W. F. Valle and A. Perez, p. 7-18

[69] B.T. Cleveland et al., Nucl. Phys. B (Proc. Suppl.) 38 (1995) 47.

[70] Y. Suzuki, Nucl. Phys. B (Proc. Suppl.) B38 (1995) 54 
[71] GALLEX Collaboration, P. Anselmann et al., LNGS Report 95/37 (June 1995).

[72] SAGE Collaboration, J.S. Nico et al., Proc. 27th Conf. on High Energy Physics, Glasgow, UK (July 1994).

[73] J. N. Bahcall and R. K. Ulrich, Rev. Mod. Phys. 60 (1990) 297; J. N. Bahcall and M. H. Pinsonneault, Rev. Mod. Phys. 64 (1992) 885; J. N. Bahcall and M. H. Pinsonneault, preprint IASSNS-AST 95/24 S. Turck-Chiéze and I. Lopes, Ap. J. 408 (1993) 346; S. Turck-Chiéze et al., Phys. Rep. 230 (1993) 57.

[74] V. Castellani, et al Phys. Lett. B324 (1994) 245; J. N. Bahcall, Phys. Lett. B338 (1994) 276; N. Hata, S. Bludman, and P. Langacker, Phys. Rev. D49 (1994) 3622; V. Berezinsky, Comments on Nuclear and Particle Physics 21 (1994) 249

[75] Z. Berezhiani, A. Rossi, Phys. Rev. D51 (1995) 5229, Phys. Lett. B367 (1996) 219. Previous references can be found in this paper.

[76] M. Mikheyev, A. Smirnov, Sov. J. Nucl. Phys. 42 (1986) 913; L. Wolfenstein, Phys. Rev. D17 (1978) 2369; ibid. D20 (1979) 2634.

[77] H. Nunokawa, A. Rossi, V. Semikoz and J. W. F. Valle, hep-ph/9602307

[78] F. N. Loreti and A. B. Balantekin, Phys. Rev. D50 (1994) 4762

[79] See, e.g. X. Shi, D. N. Schramm, B. D. Fields, Phys. Rev. D48 (1993) 2563.

[80] C. Arpasella et al, Borexino proposal, 1991.

[81] B. Barish, in proceedings of Int. Workshop on Elementary Particle Physics: Present and Future, ed. A. Ferrer and J. W. F. Valle, World Scientific, in press; talk by T. Stanev at TAUP95, Toledo, Sept. 1995.

[82] A. Suzuki, talk at SUSY95, Paris, 1995, preprint TOHOKU-HEP-95-03

[83] D.O. Caldwell and R.N. Mohapatra, Phys. Rev. D48 (1993) 3259; A. S. Joshipura, preprint PRL-TH/93/20, December 1993, to appear in Zeit. fur Physik (1994) ; S. T. Petcov, A. Smirnov, Phys. Lett. B322 (1994) 109

[84] A. Ioannissyan, J.W.F. Valle, Phys. Lett. B332 (1994) 93-99; B. Bamert, C.P. Burgess, Phys. Lett. B329 (1994) 289; D. Caldwell and R. N. Mohapatra, Phys. Rev. D50 (1994) 3477; D. G. Lee and R. N. Mohapatra, Phys. Lett. B329 (1994) 463; A. S. Joshipura, Phys. Rev. D51 (1995) 1321

[85] See, for example, D. Choudhury et al Phys. Rev. D50 (1994) 3486 
[86] J. T. Peltoniemi, D. Tommasini, and J W F Valle, Phys. Lett. B298 (1993) 383

[87] J. T. Peltoniemi, and J W F Valle, Nucl. Phys. B406 (1993) 409; E. Akhmedov, Z. Berezhiani, G. Senjanovic and Z. Tao, Phys. Rev. D47 (1993) 3245.

[88] J. T. Peltoniemi, Mod. Phys. Lett. A38 (1993) 3593

[89] J. W. F. Valle, in Proceedings of Second Workshop on Tau-Lepton Physics, Ohio, ed. K. K. Gan (World Scientific, 1993); see also Third Workshop on the Charm Tau Factory, Marbella, Spain, June 1993, (World Scientific, 1994), Ed. J. Kirkby and R. Kirkby.

[90] J. Bardeen, J. Bond and G. Efstathiou, Astrophys. J. 321 (1987) 28; J. Bond and G. Efstathiou, Phys. Lett. B265 (1991) 245; M. Davis et al Nature 356 (1992) 489.

[91] For a review see talks by C. Frenk and J. Primack, proceedings of the International School on Cosmological Dark Matter, Valencia Oct.1993, (World Scientific, 1994), p. 7-18, edited by J. W. F. Valle and A. Perez, pages 65 and 81.

[92] G. Altarelli et al, Interim Report on the Physics Motivations for an Energy Upgrade of LEP2, CERN-TH/95-151 (1995); M. Carena and P. Zerwas, in ref. [97]; T. L. Barklow, S. Dawson, H. Haber and J. L. Siegrist, SLAC-PUB-95-6893 hep-ph/9505296, in Particle Physics - Perspectives and Opportunity, edited by R. Peccei et al (World Scientific, 1995); J. F. Gunion, A. Stange, and S. Willenbrock, UCD-95-28 (1995), in Electroweak Symmetry Breaking and New Physics at the TeV Scale, ed. by T. Barklow et al (World Scientific, to be published).

[93] See M. Sher, Phys. Rep. 179 (1989) 273 and references therein.

[94] G. Altarelli and G. Isidori, Phys. Lett. B337 (1994) 141; J. A. Casas, J. R. Espinosa and M. Quirós, Phys. Lett. B342 (1995) 171; J.A. Casas, J.R. Espinosa, M. Quirós and A. Riotto, Nucl. Phys. B436 (1995) 3; J.R. Espinosa and M. Quirós, Phys. Lett. B353 (1995) 257.

[95] ALEPH collaboration, CERN-PPE/96-10, submitted to Phys. Lett. B (1996) .

[96] D. Treille, in proceedings of Int. Workshop on Elementary Particle Physics: Present and Future, ed. A. Ferrer and J. W. F. Valle, World Scientific, in press.

[97] M. Carena, P. Zerwas, et al, in Higgs Physics at LEPII, CERN-96-01, hep-ph/9602250

[98] D. Denegri, in proceedings of Int. Workshop on Elementary Particle Physics: Present and Future, ed. A. Ferrer and J. W. F. Valle, World Scientific, in press.

[99] P. Q. Hung, M. Sher, hep-ph/9512313 
[100] P. Antilogus et al. [LEP Electroweak Working Group], LEPEWWG/95-02 (1995), contributions of the LEP Experiments to the 1995 International Europhysics Conference on High Energy Physics, 27 July-2 August, 1995, Brussels, Belgium; P. H. Chankowski and S. Pokorski, Phys. Lett. B356 (1995) 307; MPI-PH-95-39 hep-ph/9509207; J. Ellis, G. L. Fogli, and E. Lisi, CERN-TH/95-202 hep-ph/9507424.

[101] Z. Berezhiani, A. Yu. Smirnov, J. W. F. Valle, Phys. Lett. B291 (1992) 99.

[102] A. Lopez-Fernandez, J. Romão, F. de Campos and J. W. F. Valle, Phys. Lett. B312 (1993) 240; B. Brahmachari, A. Joshipura, S. Rindani, D. P. Roy, K. Sridhar, Phys. Rev. D48 (1993) 4224; F. de Campos et al., proceedings of Moriond94, hep-ph/9405382

[103] F. de Campos, O. J. P. Eboli, J. Rosiek, J. W. F. Valle, hep-ph/9601269.

[104] O. Eboli, M. C. Gonzalez-Garcia, A. Lopez-Fernandez, S. Novaes, and J. W. F. Valle, Nucl. Phys. B421 (1994) 65

[105] J. W. F. Valle, Nucl. Phys. B (Proc. Suppl.) 31 (1993) 221-232; J. C. Romão, F. de Campos, L. Diaz-Cruz, and J. W. F. Valle, Mod. Phys. Lett. A9 (1994) 817; J. Gunion, Phys. Rev. Lett. 72 (1994) 199; D. Choudhhury, D. P. Roy, Phys. Lett. B322 (1994) 368.

[106] F. de Campos, M. A. Garcia-Jareño, A. Joshipura, J. Rosiek, D. P. Roy, J. W. F. Valle, Phys. Lett. B336 (1994) 446-456

[107] G. G. Ross, Grand Unified Theories (Benjamin, 1984), and references therein.

[108] J. Wess, B. Zumino, Nucl. Phys. B89 (1975) 535; S. Coleman, J. Mandula, Phys. Rev. 159 (1967) 1251; R. Haag et al Nucl. Phys. B88 (1975) 257

[109] U. Amaldi, W. de Boer, H. Fursteneau, Phys. Lett. B260 (1991) 447; F. Anselmo et al, Nuovo Cim. 104A (1992) 1335; J. Ellis et al, Nucl. Phys. B373 (1992) 55, Phys. Lett. B287 (1992) 95

[110] P. Langacker, N. Polonski, Phys. Rev. D52 (1995) 3081

[111] H. Haber and G. Kane, Phys. Rep. 117 (1985) 75; H. P. Nilles, Phys. Rep. 110 (1984) 1

[112] L. Ibañez, C. Lopez, Phys. Lett. B126 (1983) 54; L. Alvarez-Gaumé, J. Polchinski and M. Wise, Nucl. Phys. B221 (1983) 495; J. Ellis, D. Nanopoulos, K. Tamvakis, Phys. Lett. B121 (1983) 123; for more recent discussion and refernces, see ref. 97, 117.

[113] R. Barbieri, S. Ferrara, and C. Savoy, Phys. Lett. B119, 343 (1982). 
[114] J. F. Gunion, H. E. Haber, G. L. Kane and S. Dawson, The Higgs Hunter's Guide (Addison-Wesley, Redwood City, CA, 1990).

[115] H. E. Haber and R. Hempfling, Phys. Rev. Lett. 66 (1991) 1815; Y. Okada, M. Yamaguchi and T. Yanagida, Prog. Theor. Phys. 85 (1991) 1; J. Ellis, G. Ridolfi and F. Zwirner, Phys. Lett. B257 (1991) 83.

[116] P. Chankowski, S. Pokorski, and J. Rosiek, Phys. Lett. B274 (1992) 191; Nucl. Phys. B423 (1994) 437; A. Dabelstein, Z. Phys. C67 (1995) 495.

[117] M. Carena, J. R. Espinosa, M. Quiros, and C. E. M. Wagner, Phys. Lett. B355 (1995) 209; J. A. Casas, J. R. Espinosa, M. Quiros, A. Riotto, Nucl. Phys. B436 (1995) 3, [E: Nucl. Phys. B439 (1995) 466]; M. Carena, M. Quiros, and C.E.M. Wagner, CERN-TH95-157 (1995).

[118] H. Haber, talk at EPS HEP Conference, Brussels hep-ph/9601330

[119] D0 collaboration, Phys. Rev. Lett. 75 (1995) 618; and references therein, CDF collaboration, Phys. Rev. Lett. 69 (1992) 3439; UA2 collaboration, Phys. Lett. B235 (1990) 363.

[120] R. Barbieri, G. Giudice, Nucl. Phys. B306 (1988) 63

[121] P. Nogueira, J. C. Romão, J. W. F. Valle, Phys. Lett. B251 (1990) 142; R. Barbieri, L. Hall, Phys. Lett. B238 (1990) 86. M. C. Gonzalez-Garcia, J. W. F. Valle, Nucl. Phys. B355 (1991) 330

[122] L. Hall, M. Suzuki, Nucl. Phys. B231 (1984) 419; S. Dimopoulos, L.J. Hall, Phys. Lett. 207B (1988) 210; E. Ma, D. Ng, Phys. Rev. D41 (1990) 1005

[123] V. Barger, G. F. Giudice, T. Han, Phys. Rev. D40 (1989) 2987.

[124] D. Buskulic et al Phys. Lett. B349 (1995) 238

[125] B. A. Campbell, S. Davison, J. Ellis, K. Olive, Phys. Lett. B256 (1991) 457; W. Fischler, G.F. Giudice, R.G. Leigh, S. Paban, Phys. Lett. B258 (1991) 45.

[126] R. Mohapatra, J. W. F. Valle, Phys. Lett. B186 (1987) 303; S. Dimopoulos, L.J. Hall, Phys. Lett. 196B (1987) 135; J. Cline, S. Raby, Phys. Rev. D43 (1991) 1781-1787

[127] A Masiero, J. W. F. Valle, Phys. Lett. B251 (1990) 273; J. C. Romão, C. A. Santos, and J. W. F. Valle, Phys. Lett. B288 (1992) 311

[128] G. Giudice, A. Masiero, M. Pietroni, A. Riotto, Nucl. Phys. B396 (1993) 243 ; M. Shiraishi, I. Umemura, K. Yamamoto, Phys. Lett. B313 (1993) 89; see also I. Umemura, K. Yamamoto, Nucl. Phys. B423 (1994) 405 
[129] J. W. F. Valle, Phys. Lett. B196 (1987) 157

[130] M. C. Gonzalez-Garcia, J. W. F. Valle, Nucl. Phys. B355 (1991) 330.

[131] F. de Campos, M. A. Garcia-Jareño, A. Joshipura, J. Rosiek, J. W. F. Valle, Nucl. Phys. B451 (1995) 3-15

[132] J. E. Kim, Phys. Rep. 150 (1987) 1 D. Dearborn, et al., , Phys. Rev. Lett. 56 (1986) 26; M. Fukugita et al., , Phys. Rev. Lett. 48 (1982) 1522; Phys. Rev. D26 (1982) 1841; J. Ellis, K. Olive, Nucl. Phys. B223 (1983) 252

[133] M. C. Gonzalez-Garcia, J. C. Romão, J. W. F. Valle, Nucl. Phys. B391 (1993) 100

[134] J. C. Romão, F. de Campos, and J. W. F. Valle, Phys. Lett. B292 (1992) 329.

[135] V. Berezinskii and J. W. F. Valle, Phys. Lett. B318 (1993) 360; A. D. Dolgov, S. Pastor, and J. W. F. Valle astro-ph/9506011

[136] F. de Campos, M. A. Garcia-Jareño, M. B. Magro, J. Romão, J. W. F. Valle, in preparation; M. Pimenta et al, in preparation

[137] J. Romão, J. Rosiek and J. W. F. Valle, Phys. Lett. B351 (1995) 497

[138] J. C. Romão, N. Rius, J. W. F. Valle, Nucl. Phys. B363 (1991) 369. 